\title{
Wavelength Tuning of the Soft-approached Whispering Gallery Mode Microlasers for Display and Sensing
}

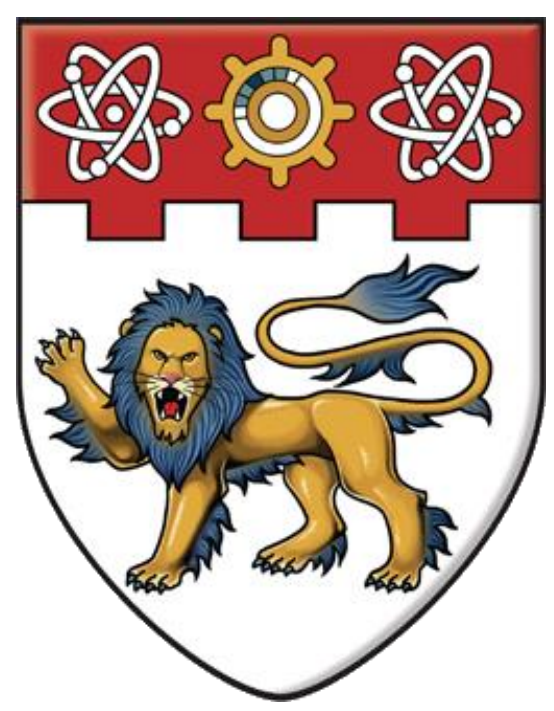

YANG SHANCHENG

DIVISION OF PHYSICS AND APPLIED PHYSICS

SCHOOL OF PHYSICAL AND MATHEMATICAL SCIENCES

NANYANG TECHNOLOGICAL UNIVERSITY 



\title{
Wavelength Tuning of the Soft-approached Whispering Gallery Mode Microlasers for Display and Sensing
}

\author{
YANG SHANCHENG \\ Division of Physics and Applied Physics \\ School of Physical and Mathematical Sciences
}

A thesis submitted to the Nanyang Technological University In partial fulfillment of the requirement for the degree of Doctor of Philosophy 



\section{Table of Contents}

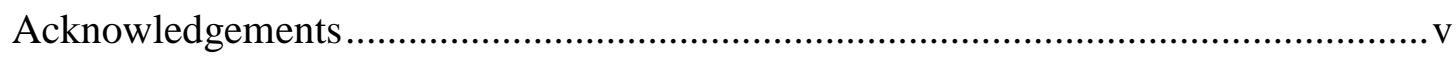

List of Publications ............................................................................................

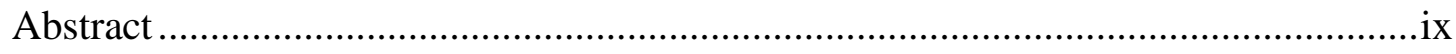

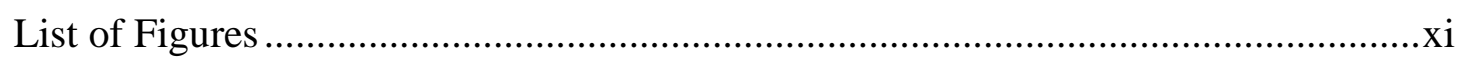

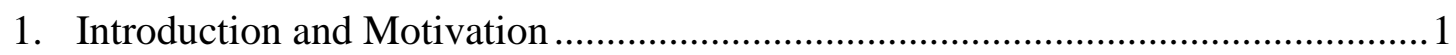

1.1. The significance of microcavity studies .................................................. 1

1.2. Basis of the whispering gallery mode microcavities and microlasers .............. 3

1.2.1. The configurations of microcavities and WGM microcavities ..........3

1.2.2. The passive WGM microresonators and applications ......................5

1.2.3. The active WGM microlasers and dye-laser fundamentals ..............6

1.3. Whispering gallery mode characterization ............................................... 9

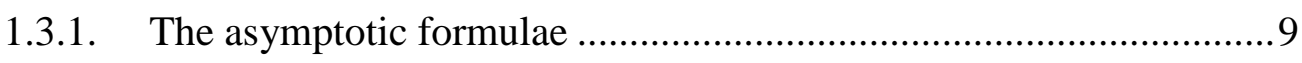

1.3.2. The characteristic equations ...................................................... 12

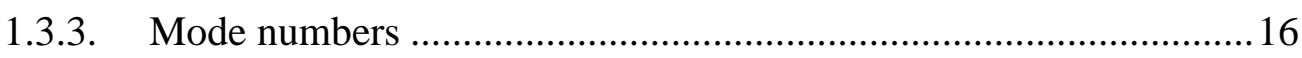

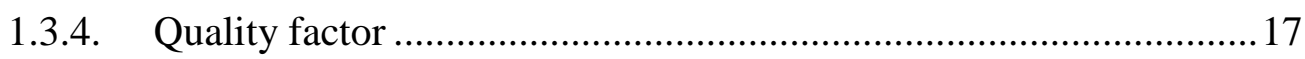

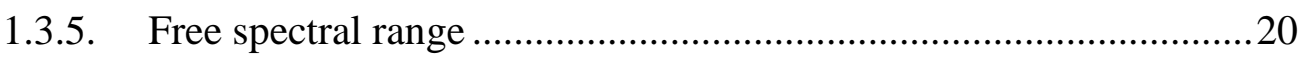

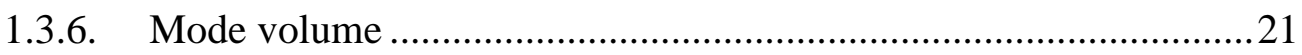

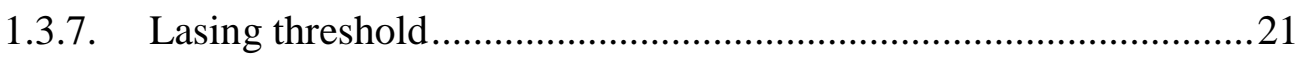

1.4. The advances of the whispering gallery mode microcavities and microlasers... 
1.4.1. Processing of the glassy candidates

1.4.2. Top-down and bottom-up of semiconductor processing .................24

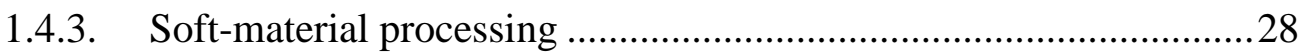

1.5. Wavelength tuning of the whispering gallery modes ................................... 32

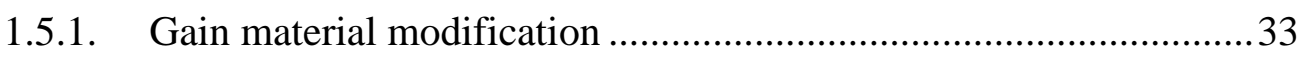

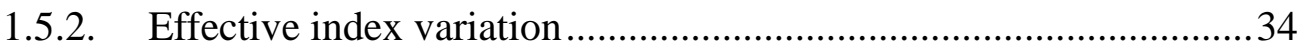

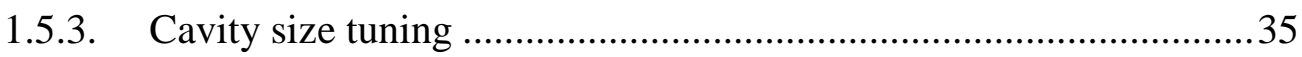

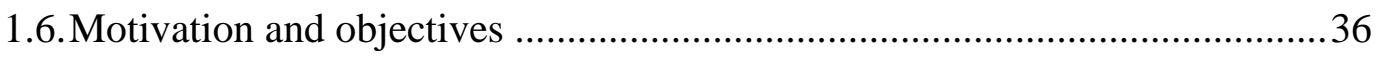

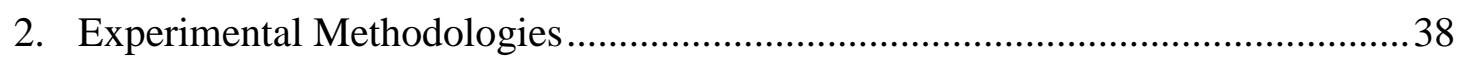

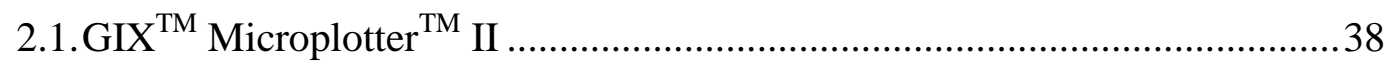

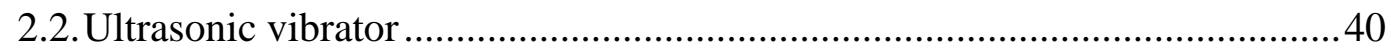

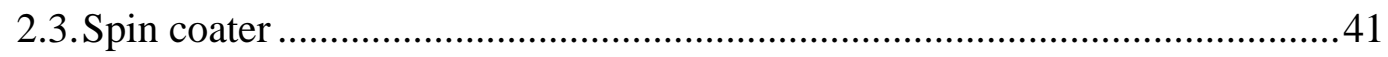

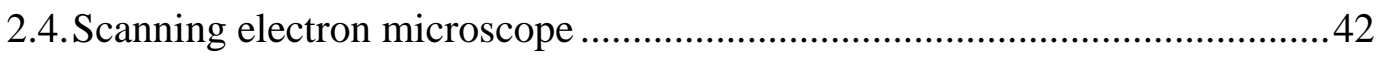

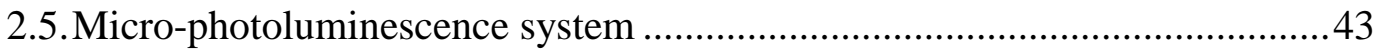

2.6. Time-resolved photoluminescence measurement ....................................... 45

3. Printable and Multicolor Whispering Gallery Mode Hemispherical Lasers .........46

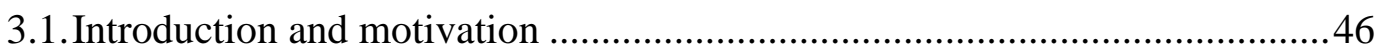

3.2.Inkjet printing of the dye-doped hemispherical microlasers ........................47

3.3. Multicolor lasing from the dual-dye-doped hemispheres ............................51

3.4.Investigation of the energy transfer processes ..........................................55

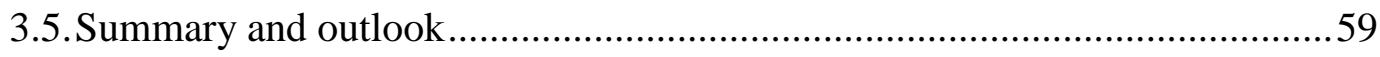

4. Spirally Drawn Whispering Gallery Mode Microfiber Arrays and Sensing 
4.2.Spiral drawing of the microfiber lasers

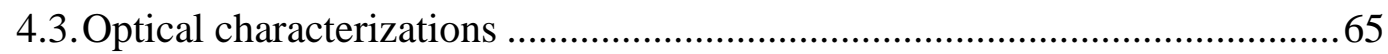

4.4. Mechanical tuning of the microfibers and the sensing applications..............69

4.5.The advantages of the microfiber array sensors in PDMS ...........................74

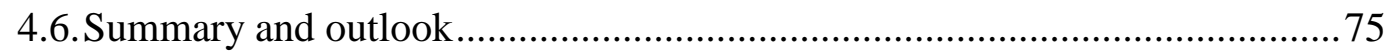

5. Reconfigurable Tuning of Liquid Whispering Gallery Mode Microlasers via

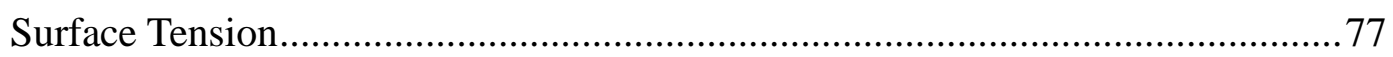

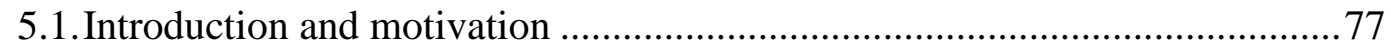

5.2.Quasi-disk floating microlaser fabricated by inkjet printing .......................78

5.3. Whispering gallery mode lasing characterization.................................... 80

5.4. Tuning mechanism of the floating microlasers........................................ 82

5.5.Reconfigurable wavelength tuning of the microlasers through environmental

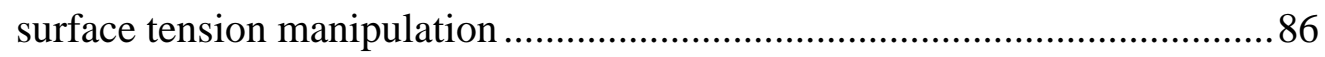

5.6. Water-soluble organic compound sensing ................................................ 91

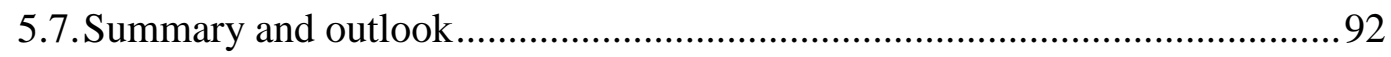

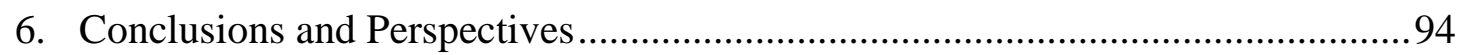

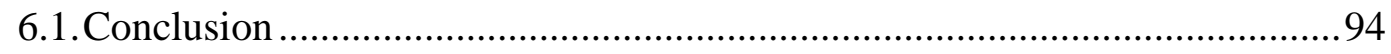

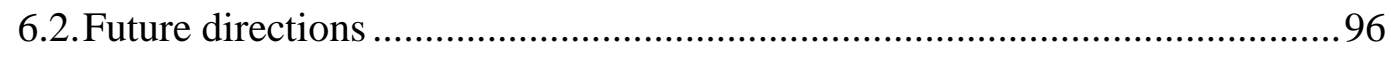

6.2.1. High-efficiency coupling of the polymer waveguides and WGM microlasers . .96 
6.2.2. Unidirectional emission of the WGM microlasers .97

6.2.3. Symmetry breaking and its applications in soft-approached WGM microcavities and microlasers .99

6.2.4. Electrically excited soft microlasers 100

Bibliography 102 


\section{Acknowledgements}

I would like to thank all the people who supported me with guidance, encouragement and love during the past four years.

First of all, I need to thank my supervisor, Prof. Sun Handong, who gave me tremendous guidance and support in my academic career. I really benefit a lot from the insightful discussions with him. Meanwhile, the harmonious and rigorous scientific atmosphere in Prof. Sun's group is impressive and always motivates me to explore the unknown physical world.

I also thank my co-supervisor Prof. Hilmi Volkan Demir for the generous support and encouragement. The discussions and meetings with him were always enlightening and cheerful.

Next, I would like to give my gratefulness to Dr. Ta Van Duong, who is the former member of our group. His is a nice teacher with profound knowledge and great patience. Without his tolerance and guidance, I cannot fulfill the works in this thesis. Wish him all the best in the future.

Then I thank all my group members and friends for the guidance, help, discussion, and the unforgettable time spent with them: Dr. Chen Rui, Dr. He Tingchao, Dr. Xu Hongyi, Dr. Ma Lin, Dr. Wang Yue, Dr. Gao Yuan, Dr. Zhao Xin, Mr. Wang Zeng, Mr. Xiao Lian, Mr. Lim Wenxiang, Mr. Cheng Shijia, Ms. Chen Xiaoxuan, Ms. Wang Lin, Mr. Eugene Tay Yong Kang, Mr. Li Zhenpeng, Mr. Zhang Chiyuan, Dr. Zhan Yi, Dr. Li Liang, Ms. Chen Bing and Ms. Qu Jing. 
Last but not least, I want to express my sincere appreciation to my father Mr. Yang Yong, my mother Ms. Han Jing and my girlfriend Ms. Chen Kunning for their invaluable love, encouragement and support, which help me to overcome the difficulties in my scientific career and everyday life. To them, I devote the rest of my life.

December 2016, Singapore 


\section{List of Publications}

[1] Y. Wang, S. Yang, H. Yang, H. D. Sun, Quaternary Alloy Quantum Dots: Toward Low-Threshold Stimulated Emission and All-Solution-Processed Lasers in the Green Region, Advanced Optical Materials, 3, 652 (2015)

[2] Y. Wang, K. E. Fong, S. Yang, V. D. Ta, Y. Gao, Z. Wang, V. Nalla, H. V. Demir, H. D. Sun, Unraveling the ultralow threshold stimulated emission from $\mathrm{CdZnS} / \mathrm{ZnS}$ quantum dot and enabling high-Q microlasers, Laser \& Photonics Reviews, 9, 507 (2015)

[3] S. Yang, Y. Wang, H. D. Sun, Advances and Prospects for Whispering Gallery Mode Microcavities, Advanced Optical Materials, 3, 1136 (2015)

[4] V. D. Ta, S. Yang, Y. Wang, Y. Gao, T. He, R. Chen, H. V. Demir, H. D. Sun, Multicolor lasing prints, Applied Physics Letters, 107, 221103 (2015)

[5] S. Yang, V. D. Ta, Y. Wang, R. Chen, T. He, H. V. Demir, H. D. Sun, Reconfigurable Liquid Whispering Gallery Mode Microlasers, Scientific Reports, 6, $27200(2016)$

[6] S. Yang, T. Y. K. Eugene, Y. Wang, X. Zhao, H. V. Demir, H. D. Sun, Wavelength tuning of the spirally drawn whispering gallery mode microfiber lasers and the 
perspectives for sensing applications, Optics Express, 25, 2618 (2017)

Note: Chapters 1, 3, 4, 5 are based on [3], [4], [6] and [5], respectively. 


\section{Abstract}

Whispering gallery mode (WGM) microcavities and microlasers have attracted enormous research attentions in recent years due to the high quality factors, small mode volumes, enhanced light-matter interactions, and abundant applications in various fields such as optoelectronics, biological and chemical sensing, high-quality lasers, nonlinear studies, etc. Although the mature but sophisticated top-down and bottom-up approaches for semiconductor processing can provide WGM microcavities and microlasers with high quality and ultra-compact integration, the intrinsic rigid nature of the materials hinders the development of flexible applications like display and sensing, which require the tuning of the wavelengths within the cavity. As the confined resonances are ultra-sensitive to the gain medium, the refractive index and the cavity size, doping-flexible, elastic and cost-efficient soft-approached WGM microlasers are competitive in wavelength tuning and promising in application broadening. However, though the reported soft candidates based on polymer materials have shown outstanding optical performances and improved flexibility, the lack of controllable fabrication techniques dramatically decreases the practical values of such microlasers. Therefore, engineering soft-approached WGM microlasers with controllable approaches and enhanced flexibilities in wavelength tuning for practical applications remains challenging. In this thesis, three works that emphasize the challenges are introduced. By employing a commercially available microplotter, the proposed fabrication processes for the hemispheres and microfibers are well 
controlled. In addition, wavelength tuning for new applications in display and sensing are demonstrated by manipulating the gain medium and the cavity geometry of the two configurations, respectively. Furthermore, a novel floating quasi-disk microlaser, which is bi-directionally and reconfigurably tunable, is proposed and exploited as a sensitive sensor for water-soluble chemicals in microfluidics. The proposed works not only broaden the applications for the tunable microlasers but are also significant in the spread of the soft-approached WGM microcavities for industrialization and commercialization. 


\section{List of Figures}

Figure 1.1. Schematic of a laser. ...............................................................................

Figure 1.2. Microcavity configurations..................................................................

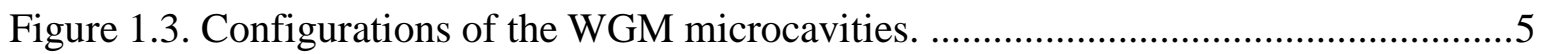

Figure 1.4. Illustration of a passive WGM microresonator. ...........................................6

Figure 1.5. Energy states of the organic dye molecules. ..............................................

Figure 1.6. The confinement and reinforcement of light inside a WGM microcavity. ........10

Figure 1.7. Coordinates in a microsphere and a microcylinder......................................13

Figure 1.8. Illustration of the angular/longitudinal mode number $\mathrm{m}$, the radial mode number $\mathrm{r}$, and the azimuthal mode number 1 in a WGM microcavity. ..............17

Figure 1.9. The top-down approach of a microtoroid cavity with high $\mathrm{Q}$ factor. ...............25

Figure 1.10. Bottom-up approach of the $\mathrm{ZnO}$ microdisk lasers.....................................27

Figure 1.11. The soft-approached hemispherical microlasers........................................30

Figure 1.12. The polymer microfiber lasers via direct drawing technique. .......................32

Figure 1.13. Realization of wavelength tuning by multi-cavity-cooperation. .....................34

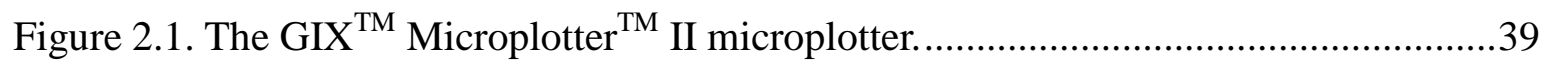

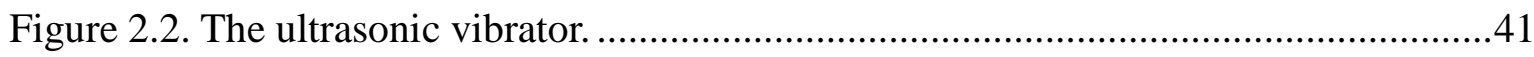

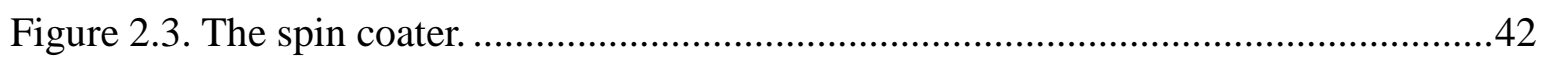

Figure 2.4. The JEOL-6700F field effect scanning electron microscope. .........................43

Figure 2.5. Schematic of the micro-photoluminescence system.....................................44

Figure 3.1. Properties of the Rhodamine B and Coumarin 540A dye molecules. ..............48 
Figure 3.2. The precise fabrication of the hemispherical microlasers.

Figure 3.3. Hemispherical microlaser arrays and the statistical distribution of the diameters.

Figure 3.4. Laser emission from the single-dye-doped hemispheres. .52

Figure 3.5. Multicolor lasing from a dual-dye-doped hemispherical microlaser. .54

Figure 3.6. Normalized PL intensities of the dual-dye-doped solutions. .55

Figure 3.7. TRPL of the donors (C540A) with and without the acceptors (RhB). .58

Figure 4.1. Illustration of the spiral drawing technique for the fabrication of microfiber arrays. .64

Figure 4.2. Analyses of the microfibers fabricated by the spiral drawing technique. .65

Figure 4.3. Excitation and collection of the microfiber laser. .66

Figure 4.4. Optical performances of the microfiber lasers .68

Figure 4.5. Schematic of the buried microfiber lasers as strain sensors. .70

Figure 4.6. Spectra of the tuned lasing peaks with respect to the elongation of the PDMS matrix. .71

Figure 4.7. The relations between the fiber diameter, PMMA to epoxy mass ratio, and the sensitivity. .73

Figure 5.1. Inkjet printing of the quasi-disk floating microlaser...... .79

Figure 5.2. The WGM lasing and characterizations of the quasi-disk microlaser. .81

Figure 5.3. The shrinkage of the quasi-disk microlasers via the modification of the liquid environment. .83

Figure 5.4. Illustration of the surface-tension-induced deformation of the floating 
quasi-disk microlaser.

Figure 5.5. The shrinkage of the horizontal cross-sections of the floating quasi-disk microlasers with various initial sizes

Figure 5.6. The microfluidic system for the measurements of wavelength tuning

Figure 5.7. Blueshift of the lasing envelope with the decrease of the cavity size .88

Figure 5.8. Analyses of the underlying physics that cause the blueshift of the lasing peaks. D refers to the diameter of the microresonator.

Figure 5.9. Reconfigurable tuning of the floating quasi-disk microlaser. .90

Figure 5.10. Sensing of the water-soluble polar chemicals.

Figure 6.1. Coupling of the polymer waveguides and WGM microlasers 97

Figure 6.2. Directional emission from the polymer WGM microlasers .98 


\section{Introduction and Motivation}

\subsection{The significance of microcavity studies}

The laser technology has experienced explosive development after the first realization of a ruby laser in 1960 , which is considered as a milestone in optics. ${ }^{[1]}$ Due to the unique properties of spatial and temporal coherence, lasers are ubiquitous nowadays, from high-powered laser weapons, laser melting and cutting, to low-powered laser pointers, barcode scanners and disk readers. In general, "laser" is the abbreviation of "Light Amplification by Stimulated Emission of Radiation" and typically contains three components, the excitation source (electrically or optically), the gain medium and the cavity (illustrated in Fig. 1.1). ${ }^{[2]}$ The gain medium amplifies the light by stimulated emission while the cavity, which is highly reflective at the boundary, confines and makes light resonate through the gain medium for feedback. Therefore, cavities with low optical loss and enhanced light-matter interaction are critical for high-quality laser devices and have attracted enormous research attention in the past decades. ${ }^{[3-5]}$

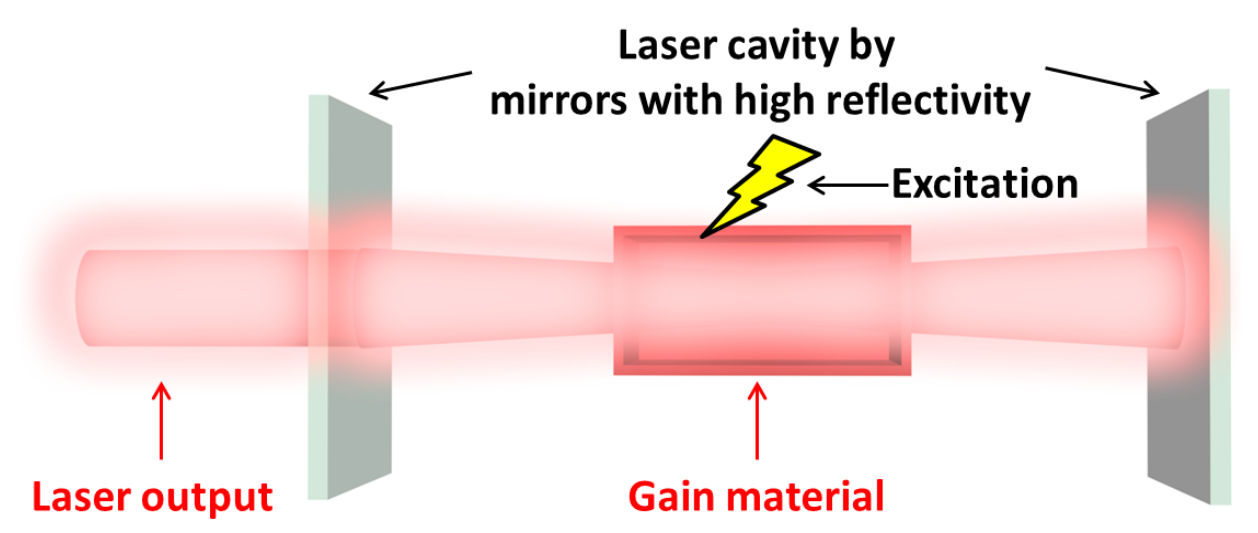

Figure 1.1. Schematic of a laser. 
With the advances in material science and processing techniques, microcavities, which are cavities in micrometer scale, are feasible and proved to be not only significant in laser evolution but also considerable in other fields. ${ }^{[6]}$ For example, in order to solve the problems of bandwidth limitation and heat generation that the conventional electron-based computing industry is facing, integrated photonic circuits or electronic/photonic hybrid circuits are highlighted. ${ }^{[7]}$ Acting as filters, switches, sources and/or memories, ${ }^{[8-11]}$ the microcavities, together with the dielectric waveguides, replace the traditional semiconductor-based elements and their metallic connection to further improve the energy efficiency and broaden the bandwidth. ${ }^{[7,12]}$ Another important property of microcavities is the ultra-sensitive response to perturbation and thus, they are promising in sensing applications. ${ }^{[6]}$ Up to now, microcavity-based sensors have shown great potential in biological sensing, chemical sensing, and microfluidic sensing. ${ }^{[13-15]}$ Meanwhile, achievements such as single molecule and single particle sensing ${ }^{[16,17]}$ further motivate scientists to explore microcavity-based sensors with more flexible configuration, higher sensitivity as well as lower limit of detection for specific sensing applications. ${ }^{[6]}$ In addition to lasing, optical elements and sensing, microcavities also play important roles in other fields like quantum information processing, nonlinear optics, material studies, etc. ${ }^{[18-21]}$ Therefore, the research of microcavity has became one of the hottest topics in the study of applied physics. 


\subsection{Basis of the whispering gallery mode microcavities and microlasers}

\subsubsection{The configurations of microcavities and WGM microcavities}

In general, the geometries of microcavities can be summerized into Fabry-Perot microcavities, photonic crystal microcavities, distributed feedback (DFB) microcavities and whispering gallery mode (WGM) microcavities (Fig. 1.2) ${ }^{[22]}$ The Fabry-Perot microcavity utilizes two mirrors with high reflectivity at the two ends of the cavity to make light oscillate inside the cavity. By creating a perturbation in a periodic photonic crystal structure, the excited local modes are trapped in the defect due to the lack of the propagation modes in the bandgap of the unperturbed area. The constructed gratings of a DFB microcavity selectively reflect the wavelengths for superposition, while the light inside a WGM microcavity is confined via total internal reflection at the circular boundary. Comparing with other configurations, the WGM microcavities hold the advantages of high quality $(Q)$ factor, low mode volume and enhanced optical density ${ }^{[6]}$ and thus, the WGM structures have been extensively explored and experienced tremendous advancement. 

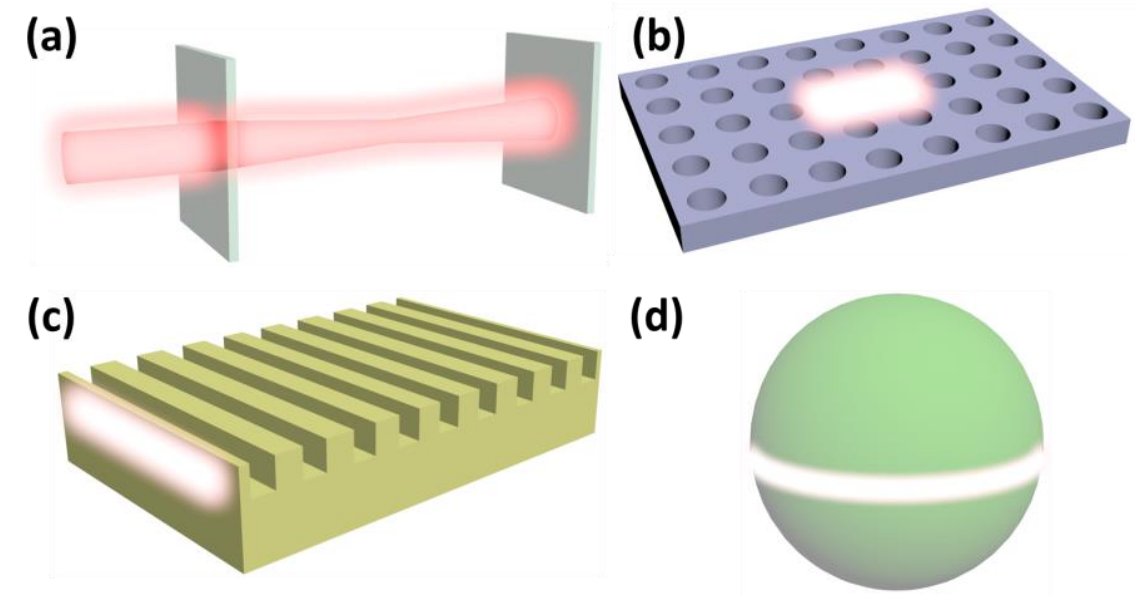

Figure 1.2. Microcavity configurations.

(a) Fabry-Perot microcavity (b) Photonic crystal microcavity. (c) Distributed feedback microcavity. (d) Whispering gallery mode microcavity. ${ }^{[6]}$

The first report of the WGM phenomenon can be traced back to 1910-1912 when Lord Rayleigh studied the dome of St Paul's Cathedral. ${ }^{[23,24]}$ He found that no matter where a person stands, the person could always hear the whispering of another as long as they both stood near the wall of the gallery (Fig. 1.3 (a)). Lord Rayleigh attributed this observation to the propagation of the acoustic wave along the circular wall of the dome. Similar propagation was then proved to be achievable in electromagnetic waves so researchers began to study the details and other possibilities of this geometry. In 1939, Richtmyer showed that high-resonance modes could be sustained in microspheres ${ }^{[25]}$ and stimulated emission in a microspherical cavity was first reported in $1961 .{ }^{[26]}$ Up to now, WGM has been proved to be sustainable in various circular structures exemplified by microspheres, ${ }^{[27]}$ hemispheres, ${ }^{[28]}$ microrings, ${ }^{[29]}$ microdisks, ${ }^{[30]}$ microtoroids, ${ }^{[31]}$ microfibers, ${ }^{[32]}$ microbottles, ${ }^{[33]}$ microbubbles, ${ }^{[34]}$ microcapillaries, ${ }^{[35]}$ etc (Fig. $1.3(\mathrm{~b}-\mathrm{j})$ ). Meanwhile, based on these WGM microcavities with different geometries and materials, abundant applications like 
optical filters, ${ }^{[36]}$ switches and memories, ${ }^{[37,38]}$ biological and chemical sensors, ${ }^{[27,39]}$ and high-quality lasers ${ }^{[10]}$ have been proposed and shown to be significant in various fields.

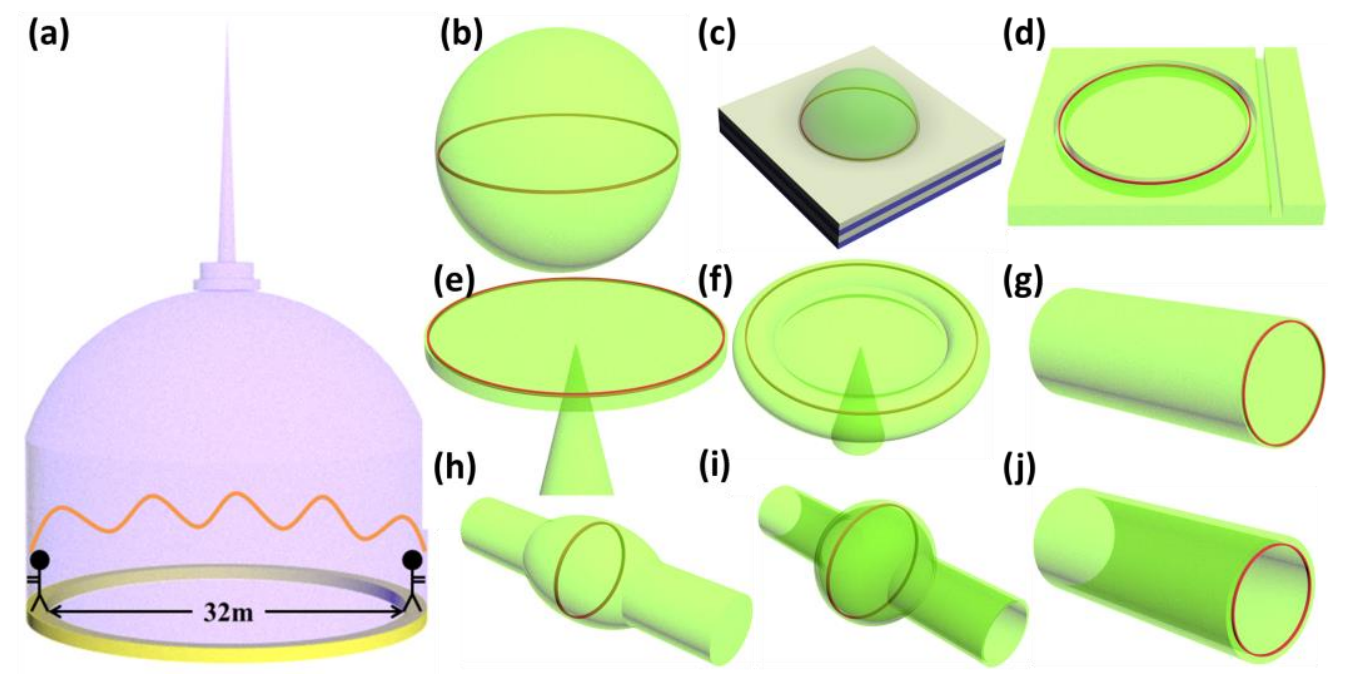

Figure 1.3. Configurations of the WGM microcavities.

(a) Schematic of the whispering gallery in St Paul's Cathedral. (b) Microsphere. (c) Hemisphere. (d) Microring. (e) Microdisk. (f) Microtoroid. (g) Microfiber. ( $h$ ) Microbottle. (i) Microbubble. (j) Microcapillary. ${ }^{[6]}$

\subsubsection{The passive WGM microresonators and applications}

The research of WGM microcavities can be divided into two parts, the passive WGM microresonators, and the active WGM microlasers. A passive WGM microresonator is typically exploited as a mode selector or filter. As illustrated in Fig. 1.4 (a), a beam of broadband light, which is transmitted through the input port of the waveguide (Fig. 1.4 (b)), can be coupled into the WGM microresonator via the small gap between the waveguide and the resonator. However, only several specific wavelengths that satisfy the resonant condition of the resonator can be trapped in the cavity while other wavelengths can be detected at the throughput port of the waveguide with low-intensity loss. So the wavelengths that are in resonance are 
filtered out in the spectrum received at the throughput port, leaving deep nulls at their positions (Fig. 1.4 (c)). At the same time, the resonant wavelengths can be further coupled out to the other waveguide and captured from the output port (Fig. 1.4 (d)). Therefore, the modes are filtered at the throughput port and selected at the output port.

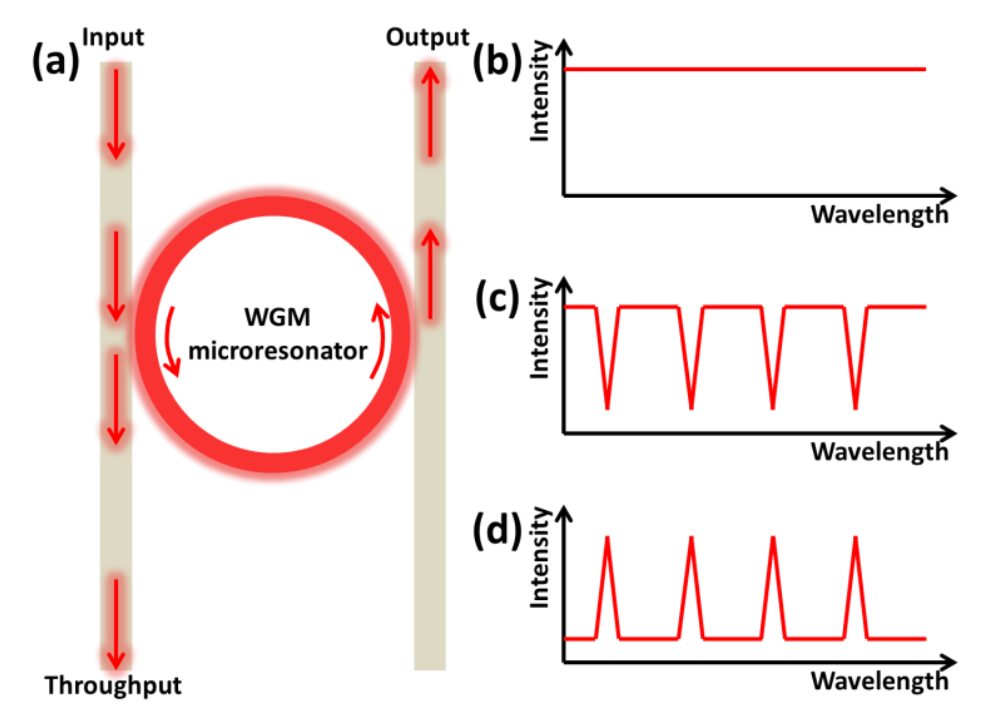

Figure 1.4. Illustration of a passive WGM microresonator.

(a) Schematic of the passive WGM microresonator (microring) system as mode selector/filter. (b) Signal imported to the input port. (c) Signal received from the throughput port. (d) Signal collected at the output port.

\subsubsection{The active WGM microlasers and dye-laser fundamentals}

After doping active gain materials into the microcavity, the passive WGM microresonators can become active WGM microlasers. The resonant condition of the resonator is not the only dominant factor in determining the position of the lasing peaks in a WGM microlaser. The emission of the gain medium also makes a big difference. Although a variety of available gain materials have been reported to be applicable for microlasers, ${ }^{[20,28,40]}$ only organic dye molecules, which hold the advantage of high tenability, are introduced here because they are relevant to the work 
in this thesis.

In the classic two-level system, an atom at ground state will absorb energy and "jump" to higher level states under excitation. The atom then decays to the ground state quickly via radiative or non-radiative processes due to the instability of the excited state. The radiative process, which is also referred as spontaneous emission, releases a photon with random phase and direction while the non-radiative process normally transfers the energy to heat. In contrast, the stimulated emission, which lays the foundation of lasing, occurs when there exists an incident photon that forces the excited atom decay to the ground state in a radiative evolution and generates two photons with the same wavelength, direction, phase, and polarization. ${ }^{[2]}$ However, this two-level system is not enough to explain the dynamics in dye lasers because of the complexity of the energy bands of the dye molecules (Fig. 1.5) ${ }^{[41]}$ In the advanced multi-level system, the molecules initially "jump" from the ground state, which is denoted as level 0 in $S_{0}$, to higher singlet states (level 3 in $S_{1}$ ) under excitation. Then they quickly relax to level 2 , which is the lower excited state in $S_{1}$. Due to the instability of the excited states, they can relax to the lower singlet energy state (level 1 in $\mathrm{S}_{0}$ ) through spontaneous emission or stimulated emission and further decay to level 0 in $\mathrm{S}_{0}$ by non-radiative processes. The existence of intersystem crossing also enables the molecules at level 2 to relax to the $\mathrm{T}_{1}$, which is the lower level of the triplet states. Then molecules at $T_{1}$ can be released to level 1 in $S_{0}$ via radiative or non-radiative processes and finally reach the ground state (level 0 in $\mathrm{S}_{0}$ ). 


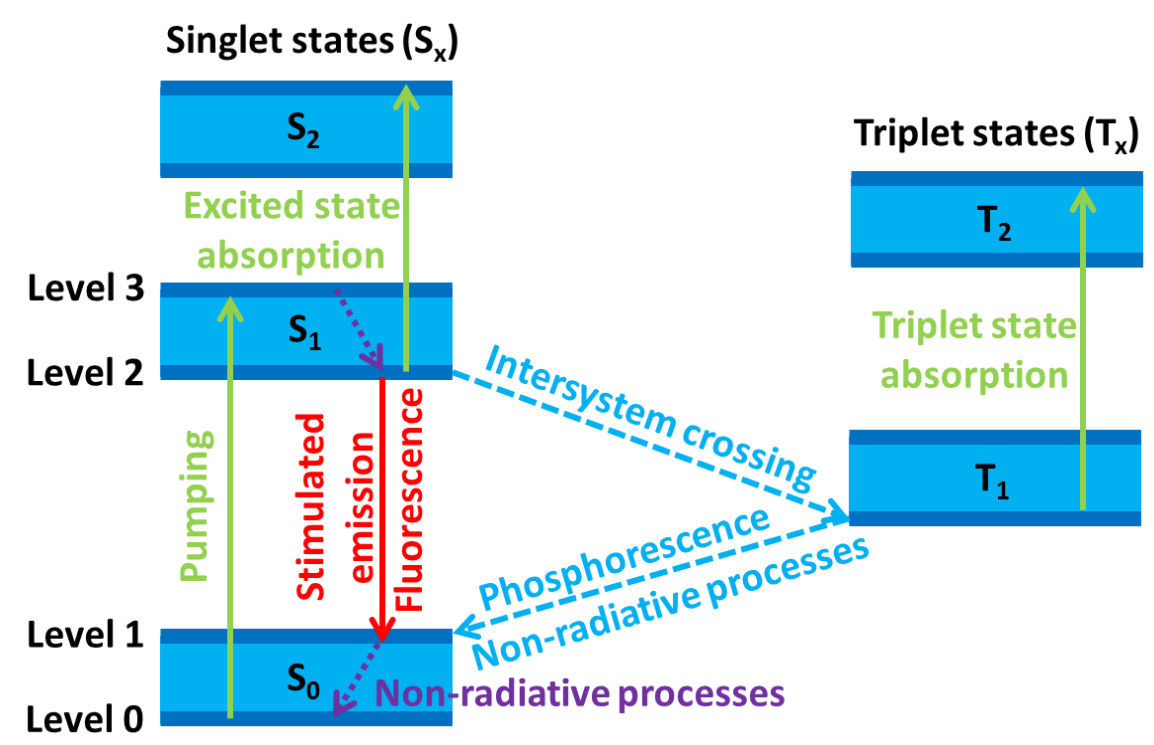

Figure 1.5. Energy states of the organic dye molecules. ${ }^{[41]}$

It is also possible that the molecules at the excited singlet state $\left(\mathrm{S}_{1}\right)$ and lower triplet states $\left(T_{1}\right)$ continuously absorb energy to reach higher energy states $\left(S_{2}\right.$ and $\left.T_{2}\right)$, which are known as excited state absorption and triplet state absorption, respectively. However, the excited state absorption and triplet state absorption are normally suppressed during the excitation processes ${ }^{[42]}$ so the dye molecules are considered to be mostly distributed in the ground state (level 0 in $S_{0}$ ), the lower level of the excited singlet state (level 2 in $S_{1}$ ) and the lower level of the triplet states $\left(\mathrm{T}_{1}\right)$. Accordingly, the rate equations can be expressed as: $:^{[43]}$

$$
\begin{gathered}
\frac{d P_{S 0}}{d t}+\frac{d P_{S 1}}{d t}+\frac{d P_{T 1}}{d t}=0 \\
\frac{d P_{S 0}}{d t}=\frac{P_{S 1}}{\tau}+P_{S 1} I_{s t i m u} B_{10}+\frac{P_{T 1}}{\tau_{T}}-P_{S 0} R_{e x t} B_{01} \\
\frac{d P_{S 1}}{d t}=-\frac{P_{S 1}}{\tau}-P_{S 1} I_{\text {stimu }} B_{10}-P_{S 1} B_{S T}+P_{S 0} R_{e x t} B_{01} \\
\frac{d P_{T 1}}{d t}=P_{S 1} B_{S T}-\frac{P_{T 1}}{\tau_{T}}
\end{gathered}
$$

where $P_{S 0}, P_{S 1}$ and $P_{T 1}$ represent the population of dye molecules in each energy 
state, $\tau$ and $\tau_{T}$ stand for the lifetime of spontaneous emission and the decay time of the triplet state, $I_{\text {stimu }}$ is the intensity of the stimulated emission or laser, $R_{\text {ext }}$ is the excitation rate, $B_{10}, B_{01}$ and $B_{S T}$ are the Einstein coefficients of $S_{1}$ (level 2) to $S_{0}$ (level 1), $S_{0}$ (level 0) to $S_{1}$ (level 3), and rate constant of the decay from $S_{1}$ (level 2) to $\mathrm{T}_{1}$, respectively. The rate equations denote the consistency of the population of dye molecules and the dynamics between the energy states. However, lasing is not only related to the stimulated emission of the dye molecules, but also critical to the microcavity. Set $\tau_{W G M}$ as the lifetime of the photon within the WGM microlaser and then,

$$
\frac{d I_{\text {stimu }}}{d t}=P_{S 1} I_{\text {stimu }} B_{10}-\frac{I_{\text {stimu }}}{\tau_{W G M}} .
$$

This means that lasing can only occur when the rate of stimulated emission is higher

than the rate of loss within the WGM microlaser. The point at which $\frac{d I_{\text {stimu }}}{d t}=0$ is referred as the lasing threshold of the microlaser.

\subsection{Whispering gallery mode characterization}

\subsubsection{The asymptotic formulae}

It is known that the WGM microcavities and microlasers confine light via total internal reflection at the circular boundary (Fig. 1.6). However, light, which phases are different at the same point after traveling one round trip, is suppressed and eliminated via self-superposition. Only the light that satisfies the resonant condition of the microcavity, which is denoted as:

$$
m \lambda=L n_{e f f}
$$


can be reinforced after propagating thousands of round trips. Here, $m$ is an integer, which is also called longitudinal or angular mode number, $\lambda$ is the wavelength of the light, $L$ is the circumference of the circular WGM boundary and $n_{\text {eff }}$ is the effective index. The self-enhanced wavelengths are referred as whispering gallery modes (WGMs, the bright spots in Fig. 1.6) and Eq. (1.6) is the basic description of the WGMs and WGM microcavities.

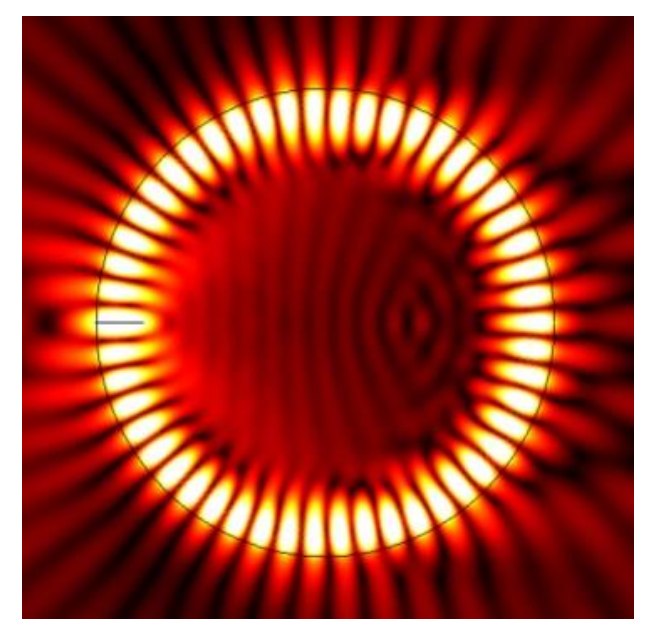

Figure 1.6. The confinement and reinforcement of light inside a WGM microcavity.

In 1992, the following asymptotic formulas were developed by Lam et al. for a more precise description of the resonant modes, which contain the correction terms of the transverse electric (TE) modes and transverse magnetic (TM) modes. ${ }^{[44-46]}$

$$
\lambda^{-1}\left(R, n_{1}, n_{r}, r, m\right)=\frac{1}{2 \pi R n_{1}}\left[\begin{array}{c}
m+\frac{1}{2}+2^{-\frac{1}{3}} a(r)\left(m+\frac{1}{2}\right)^{\frac{1}{3}} \\
-\frac{L}{\left(n_{r}^{2}-1\right)^{\frac{1}{2}}}+\frac{3}{10} 2^{-\frac{2}{3}} a^{2}(r)\left(m+\frac{1}{2}\right)^{-\frac{1}{3}} \\
-2^{-\frac{1}{3}} L\left(n_{r}^{2}-\frac{2}{3} L^{2}\right) \frac{a(r)\left(m+\frac{1}{2}\right)^{-\frac{2}{3}}}{\left(n_{r}^{2}-1\right)^{\frac{3}{2}}}
\end{array}\right]
$$




$$
n_{r}=\frac{n_{1}}{n_{2}}, \quad \mathrm{~L}=\left\{\begin{array}{l}
n_{r} \text { for } T E \text { modes } \\
\frac{1}{n_{r}} \text { for } T M \text { modes }
\end{array}\right.
$$

where $R$ is the radius of the circular microcavity, $n_{1}$ is the refractive index of the cavity, $n_{2}$ is the refractive index of the environment, $r$ is the radial mode number, and $a(r)$ is the solution of the Airy function. It is important to note that the fundamental modes, which are frequently observed in experiments, ${ }^{[47,48]}$ can be derived from Eq. (1.7) by setting $r=1$. Meanwhile, the terms $\left(m+\frac{1}{2}\right)^{-\frac{1}{3}}$ and $\left(m+\frac{1}{2}\right)^{-\frac{2}{3}}$ are negligible in simplification as the angular mode number $m$ is in the order of $10^{2}$ to $10^{3}$ in real cases. Therefore, substituting $a(r)=2.338$ and $n_{2}=1$ we have ${ }^{[6]}$

$$
\begin{gathered}
\lambda_{T E} \approx \frac{2 \pi R n_{1}}{m+1.856 m^{\frac{1}{3}}+\left(\frac{1}{2}-\frac{n_{1}}{\sqrt{n_{1}^{2}-1}}\right)}, \\
\lambda_{T M} \approx \frac{2 \pi R n_{1}}{m+1.856 m^{\frac{1}{3}}+\left(\frac{1}{2}-\frac{1}{n_{1} \sqrt{n_{1}^{2}-1}}\right)},
\end{gathered}
$$

Eq. (1.8) and Eq. (1.9) are simplified approximations of the WGM fundamental TE and TM modes based on the assumption that the surrounding material of the microcavity is air. In order to achieve total internal reflection, the prerequisite of a WGM microcavity is that the refractive index of the cavity $\left(n_{1}\right)$ is larger than the index of the surrounding material $\left(n_{2}\right)$. So $n_{1}>1$ and $\frac{\mathrm{n}_{1}}{\sqrt{\mathrm{n}_{1}^{2}-1}}$ is larger than $\frac{1}{\mathrm{n}_{1} \sqrt{\mathrm{n}_{1}^{2}-1}}$, which means that the wavelengths of TE resonances are longer than the ones of TM modes for the same angular/longitudinal mode number $m$. 


\subsubsection{The characteristic equations}

In fact, there exist multiple circular planes in the majority of WGM microresonators such as microspheres, hemispheres, microbottles, and microbubbles. The fundamental modes are typically located along the equator of these structures due to the relatively low optical loss. However, other circular planes can also sustain WGM modes so in order to fully reveal the field distributions inside a WGM microcavity, the three-dimensional model is set up and the characteristic equations are derived. As shown in Fig. 1.7 (a), the electromagnetic light wave propagates in a dielectric microsphere in a zigzag path. For simplification, the free electric charge density and the free current density are all set to be zero and therefore, the Maxwell's equations are transferred into: $:^{[49]}$

$$
\begin{gathered}
\boldsymbol{\nabla} \cdot \boldsymbol{E}(\boldsymbol{r}, t)=0, \\
\boldsymbol{\nabla} \cdot \boldsymbol{B}(\boldsymbol{r}, t)=0 \\
\boldsymbol{\nabla} \times \boldsymbol{E}(\boldsymbol{r}, t)=-\frac{1}{c} \partial_{t} \boldsymbol{B}(\boldsymbol{r}, t), \\
\boldsymbol{\nabla} \times \boldsymbol{B}(\boldsymbol{r}, t)=\frac{1}{c^{2}} \partial_{t} \boldsymbol{\varepsilon} \boldsymbol{E}(\boldsymbol{r}, t),
\end{gathered}
$$

where $\boldsymbol{E}(\boldsymbol{r}, t)$ and $\boldsymbol{B}(\boldsymbol{r}, t)$ represent the electric fields and magnetic fields, respectively, with respect to the spatial vector $\boldsymbol{r}$ and time $t$. Substituting Eq. (1.13) into Eq. (1.12) and take the curl of the resulted equation leads to:

$$
\boldsymbol{\nabla} \times \boldsymbol{\nabla} \times \boldsymbol{E}(\boldsymbol{r}, t)=\frac{n^{2}}{c^{2}} \partial_{t} \boldsymbol{E}(\boldsymbol{r}, t) .
$$

As the electric fields can be presented as $\boldsymbol{E}(\boldsymbol{r}, t)=\boldsymbol{E}_{0} e^{i(\boldsymbol{k} \cdot \boldsymbol{r}-\omega t)}$, Eq. (1.14) can be transferred into:

$$
\nabla^{2} \boldsymbol{E}(\boldsymbol{r}, t)=-k^{2} \boldsymbol{E}(\boldsymbol{r}, t)
$$




$$
k=n \frac{\omega}{c}
$$

$k$ is the wave number, $n$ is the refractive index, $\omega$ is the angular frequency and $c$ is the speed of light in vacuum.
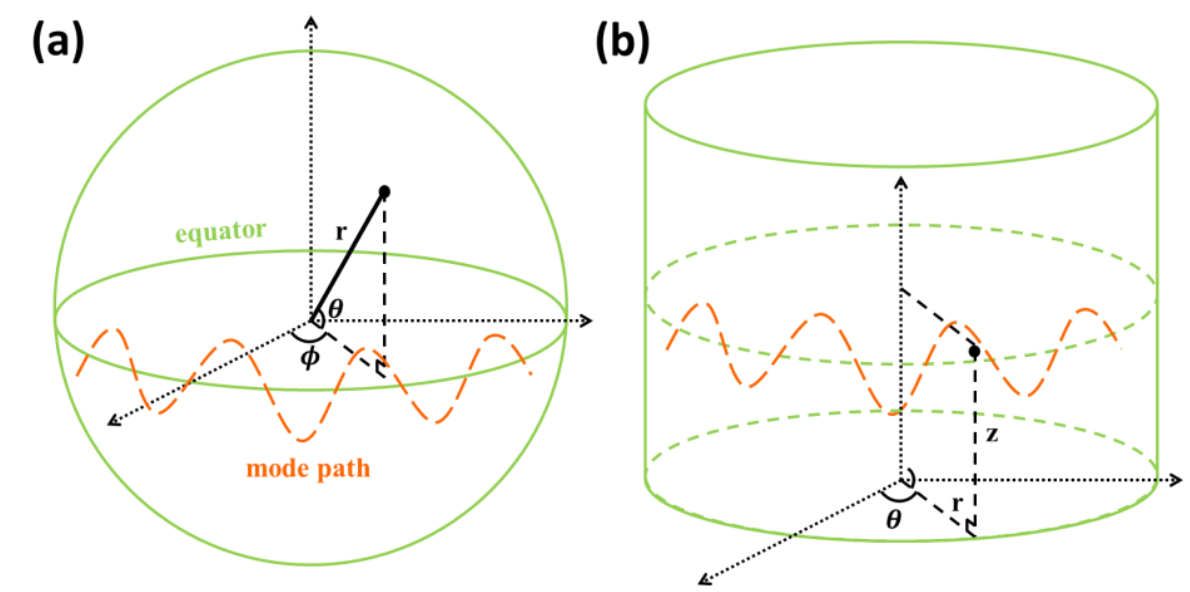

Figure 1.7. Coordinates in a microsphere and a microcylinder.

(a) Spherical coordinate in a dielectric microsphere. (b) Cylindrical coordinate in a dielectric microcylinder. ${ }^{[6,49]}$

In a spherical coordinate, the Laplacian operator " $\nabla$ " is defined as: ${ }^{[49]}$

$$
\nabla^{2}=\frac{1}{r^{2}} \frac{\partial}{\partial r}\left(r^{2} \frac{\partial}{\partial r}\right)+\frac{1}{r^{2} \sin ^{2} \phi} \frac{\partial^{2}}{\partial \theta^{2}}+\frac{1}{r^{2} \sin \phi} \frac{\partial}{\partial \phi}\left(\sin \phi \frac{\partial}{\partial \phi}\right),
$$

where $r, \theta$ and $\phi$ are the variables in the coordinate (Fig. 1.7 (a)).

Therefore, by utilizing the spherical Bessel function of the first kind and the Legendre polynomial, the intensities of the electric and magnetic fields confined inside the microsphere are expressed as:

$$
\begin{aligned}
& {\left[\begin{array}{c}
A(E)_{r}^{i n} \\
A(E)_{\theta}^{i n} \\
A(E)_{\phi}^{i n}
\end{array}\right]=\left(\begin{array}{c}
a_{r} \\
a_{\theta} \\
a_{\phi}
\end{array}\right) j_{m}\left(k_{i n} r\right) P_{m}^{l}(\cos \theta) e^{i l \phi},} \\
& {\left[\begin{array}{c}
A(B)_{r}^{i n} \\
A(B)_{\theta}^{i n} \\
A(B)_{\phi}^{i n}
\end{array}\right]=\left(\begin{array}{l}
b_{r} \\
b_{\theta} \\
b_{\phi}
\end{array}\right) j_{m}\left(k_{i n} r\right) P_{m}^{l}(\cos \theta) e^{i l \phi}}
\end{aligned}
$$

$A(E)$ and $A(B)$ are the amplitude of the electric fields and magnetic fields, $a$ and 
$b$ are coefficients, $j_{m}(x)$ is the spherical Bessel function of the first kind, $P_{m}^{l}(x)$ is the Legendre polynomial, $k_{\text {in }}$ is the wave number inside the dielectric microsphere, $m$ and $l$ are the angular/longitudinal mode number and azimuthal mode number, respectively.

As shown in Fig. 1.6, the confinement of light is not perfect at the curved boundary due to the frustrated total internal reflection (FTIR), leaving strong evanescent fields outside the microcavity. These exponentially decayed evanescent fields can be described as:

$$
\begin{aligned}
& {\left[\begin{array}{l}
A(E)_{r}^{\text {out }} \\
A(E)_{\theta}^{\text {out }} \\
A(E)_{\phi}^{\text {out }}
\end{array}\right]=\left(\begin{array}{l}
x_{r} \\
x_{\theta} \\
x_{\phi}
\end{array}\right) h_{m}\left(k_{\text {out }} r\right) P_{m}^{l}(\cos \theta) e^{i l \phi},} \\
& {\left[\begin{array}{l}
A(B)_{r}^{\text {out }} \\
A(B)_{\theta}^{\text {out }} \\
A(B)_{\phi}^{\text {out }}
\end{array}\right]=\left(\begin{array}{l}
y_{r} \\
y_{\theta} \\
y_{\phi}
\end{array}\right) h_{m}\left(k_{\text {out }} r\right) P_{m}^{l}(\cos \theta) e^{i l \phi},}
\end{aligned}
$$

where $x$ and $y$ are coefficients, $h_{m}(x)$ is the spherical Hankel function of the first kind and $k_{\text {out }}$ is the wave number outside the dielectric microsphere.

The applied boundary conditions for the microspheres are:

$$
\begin{gathered}
\left\{\begin{array}{c}
A(E)_{\theta}^{\text {in }}=A(E)_{\theta}^{\text {out }}, A(E)_{\phi}^{\text {in }}=A(E)_{\phi}^{\text {out }} \\
A(B)_{\theta}^{\text {in }}=A(B)_{\theta}^{\text {out }}, A(B)_{\phi}^{\text {in }}=A(B)_{\phi}^{\text {out }}, \\
A(E)_{r}^{\text {in }}=A(E)_{r}^{\text {out }}=0
\end{array}\right. \\
\left\{\begin{array}{c}
A(E)_{\theta}^{\text {in }}=A(E)_{\theta}^{\text {out }}, A(E)_{\phi}^{\text {in }}=A(E)_{\phi}^{\text {out }} \\
A(B)_{\theta}^{\text {in }}=A(B)_{\theta}^{\text {out }}, A(B)_{\phi}^{\text {in }}=A(B)_{\phi}^{\text {out }}, \quad \text { for } T E \text { modes } ; \\
A(B)_{r}^{\text {in }}=A(B)_{r}^{\text {out }}=0
\end{array}\right.
\end{gathered}
$$

Substituting Eq. (1.18), (1.19), (1.20), (1.21) into the boundary conditions shown in Eq. (1.22) and (1.23), the characteristic equations of the microsphere can be derived as: ${ }^{[6,49]}$ 


$$
\left\{\begin{array}{l}
\text { TE: } H_{m}^{\prime}\left(k_{\text {out }} R\right) J_{m}\left(k_{\text {in }} R\right)=n J_{m}^{\prime}\left(k_{\text {in }} R\right) H_{m}\left(k_{\text {out }} R\right) \\
\text { TM: } n H_{m}^{\prime}\left(k_{\text {out }} R\right) J_{m}\left(k_{\text {in }} R\right)=J_{m}^{\prime}\left(k_{\text {in }} R\right) H_{m}\left(k_{\text {out }} R\right)
\end{array},\right.
$$

where $H_{m}(x)=x h_{m}(x), J_{m}(x)=x j_{m}(x)$ and $\mathrm{R}$ is the radius of the microsphere.

In a dielectric cylinder with radius $R$, the Laplacian operator " $\nabla$ " is expressed by: ${ }^{[49]}$

$$
\nabla^{2}=\frac{1}{r} \frac{\partial}{\partial r}\left(r \frac{\partial}{\partial r}\right)+\frac{1}{r^{2}} \frac{\partial^{2}}{\partial \theta^{2}}+\frac{\partial^{2}}{\partial z^{2}},
$$

where $r, \theta$ and $\phi$ are the variables in the spherical coordinate (Fig. 1.7 (b)).

In order to simplify the situation, only the modes with both electric and magnetic fields independent from $\theta$ are considered. Therefore, the components of the amplitude of electric $(A(E))$ and magnetic $(A(B))$ fields inside the cylinder can be reduced to: ${ }^{[49]}$

$$
A_{z}=\left\{\begin{array}{rl}
J_{0}(\alpha r), & r \leq R, \quad \text { inside the cylinder } \\
q K_{0}(\beta r), & r \geq R,
\end{array},\right.
$$

where $A_{z}$ indicates either the electric or magnetic field. $J_{0}$ and $K_{0}$ are the Bessel function and the modified Bessel function of the first kind. $q$ is a constant derived from Maxwell boundary conditions. The substituted terms $\alpha$ and $\beta$ are given by:

$$
\gamma=\left[\frac{\omega^{2} n^{2}}{c^{2}}-k_{z}^{2}\right]^{1 / 2} ; \alpha=\left[k_{z}^{2}-k_{0}{ }^{2}\right]^{1 / 2},
$$

where $k_{z}$ is the wave number along the $z$ axis.

By applying Maxwell boundary conditions at $r=R$, the intensity field distribution inside the cylindrical microcavity can be obtained. For other WGM microcavities, the approaches of the characteristic equations are similar. By exploiting appropriate Laplacians, the inner and outer field distribution of the electric and magnetic fields are determined first. Then boundary conditions are utilized and the characteristic equations of a specific WGM microcavity can be derived. 


\subsubsection{Mode numbers}

The TE/TM eigenmodes of the microcavity, which are the theoretically infinite roots of the characteristic equations, are related to three quantum numbers: the angular/longitudinal mode number $m$, the radial mode number $r$, and the azimuthal mode number $l .{ }^{[6]}$ The angular/longitudinal mode number $m$ can be described as the number of reflections in one round trip along the circular boundary. For example, the angular/longitudinal mode number $m$ shown in Fig. 1.6 is 50 and the $m$ in Fig. 1.8 (a) is 15 . The radial mode number $r$ is defined as the sets of modes in the radial direction of the microcavity. Fig. 1.8 (a), (b) and (c) illustrate the situations of $r=1$, $r=2$, and $r=3$ with the angular/longitudinal mode number $m$ fixed at 15 . The azimuthal mode number $l$, which is usually addressed with the term $l-m+1$, indicates the sets of modes in the azimuthal direction. When $l=m$, the modes are in the equatorial plane of the spherical microresonator (Fig. 1.8 (d)). If $l>m$, it means that the modes are distributed in several planes that are in parallel with the plane of the equator (Fig. 1.8 (e) and (f)). Typically, the set of modes with the mode numbers of $r=1$ and $l=m$ are referred as fundamental modes (Fig. 1.8 (d)) while other sets of modes are called higher order modes. The fundamental modes are frequently reported due to the relatively lower optical loss than the higher order modes. ${ }^{[47,48]}$ 
(a)

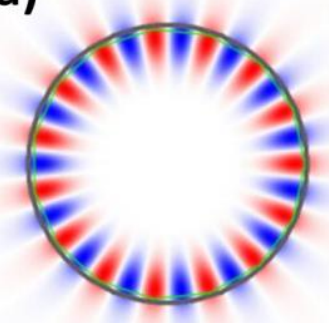

$m=15, r=1$ (b)

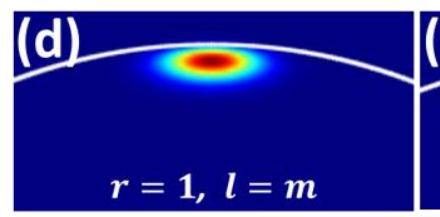

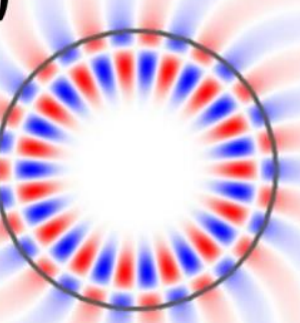

$m=15, r=2$ (c)

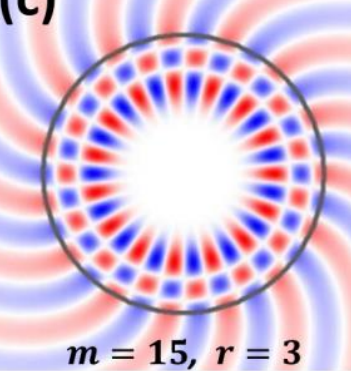

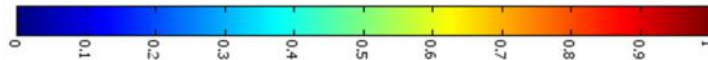

Figure 1.8. Illustration of the angular/longitudinal mode number $m$, the radial mode number $r$, and the azimuthal mode number l in a WGM microcavity. ${ }^{[6,50]}$

\subsubsection{Quality factor}

Defined as the product of the angular frequency of the modes and the ring-down lifetime of the microresonator, the quality factor, or abbreviated as $Q$ factor, illustrates the ability of light confinement of a microcavity. A large $Q$ factor means the optical loss in the microcavity is low, which indicates better energy storage and enhanced light-matter interactions. Generally, the $Q$ factor of a WGM microcavity is proportional to the scattering loss factor, the radiative loss factor, the contaminant loss factor, and the material loss factor:

$$
Q^{-1}=\sum_{i} Q_{i}^{-1}
$$

where $Q_{i}$ represents the loss factors mentioned above.

The scattering loss, which is in the form of Rayleigh scattering, is generally introduced by the surface roughness or inhomogeneity of the microcavity. ${ }^{[51]}$ During the circulation of the resonance $\lambda$, the scattering loss factor is determined by $\sigma_{r m s}$, 
the root-mean-squared size, $\mathrm{B}$, the length of inhomogeneity, and $R$, the radius of the WGM microcavity: ${ }^{[52]}$

$$
Q_{\text {scatt }}=\frac{\lambda^{2} R}{\pi^{2} \sigma_{r m s}^{2} B} .
$$

Therefore, $\sigma_{r m s}$ and $\mathrm{B}$ need to be minimized in order to achieve the maximum $Q_{\text {scatt }}$, which stands for the minimum scattering loss. By engineering the WGM microcavities with homogeneous and isotropic materials, as well as advanced processing methodologies such as self-assembling, melting and polishing, the negative effects brought by scattering loss can be reduced. Also, Eq. (1.29) shows that microcavities with large size can reduce the scattering loss and enhance the confinement of light, which is proved to be true in the reported experimental results. $^{[28]}$

Due to the existence of frustrated total internal reflection, the radiative loss is also an important factor that limits the $Q$ of a microcavity. It is understandable that the radiative loss is relatively large for light to circulate in microcavities with small diameters due to the increased curvature and the geometrically steep incident angle during total internal reflections for the same resonance. ${ }^{[6,51]}$ Theoretically, the radiative loss factor $Q_{\text {rad }}$ is defined by based on the assumption that $l=m:^{[6]}$

$$
\begin{aligned}
Q_{r a d}=\frac{\operatorname{Re}\left(k_{r, m}\right)}{\operatorname{Im}\left(k_{r, m}\right)} & \Rightarrow \frac{1}{2}\left(m+\frac{1}{2}\right) n^{(2 p-1)} \sqrt{n^{2}-1} e^{2 T_{r, m},} \\
p & =\left\{\begin{array}{l}
1 \text { for } T M \text { polarization } \\
0 \text { for } T E \text { polarization }
\end{array}\right. \\
T_{r, m} & =\left(m+\frac{1}{2}\right)\left[\beta_{r, m}-\tanh \beta_{r, m}\right]
\end{aligned}
$$




$$
\beta_{r, m}=\cosh ^{-1}\left(n\left[1-\left(\frac{1}{m+\frac{1}{2}}\right)\left(A_{r} \sqrt[3]{\frac{2 m+1}{4}}+\frac{n^{1-2 p}}{\sqrt{n^{2}-1}}\right)\right]^{-1}\right)
$$

Therefore, the angular/longitudinal mode number $m$ and the radial mode number $r$ are related closely to the radiative loss factor. It can also be derived from Eq. (1.30) and Eq. (1.31) that modes with higher order $(r>1)$ have lower value of $Q_{\text {rad }}$ than fundamental modes.

Acting as extra scatters, tiny objects such as water molecules and dust in air contribute to the contaminant loss when attached to the microcavities which are not well-protected. In addition, physically/chemically irreversible processes like oxidation brought by the contaminant of oxygen can further influence the optical performances of the microcavities. ${ }^{[53]}$ Thus, the issue of contaminant loss prevention is critical for WGM microcavities with high $Q$ factors.

From the view of materials, the absorption and scattering of the cavity material itself also induce optical energy loss within the WGM microcavity. ${ }^{[53]}$ The absorption of a linear cavity material, which is characterized by the extinction coefficient $\kappa$ and the linear absorption coefficient $\alpha$, determines the material loss factor: ${ }^{[50,54,55]}$

$$
\begin{gathered}
Q_{\text {mat }}=\frac{2 \pi n}{\alpha \lambda}, \\
n=\tilde{n}+i \kappa,
\end{gathered}
$$

where $n$ is the refractive index of the material.

The $Q$ factor is one of the most significant parameters in microcavity and microlaser studies. A WGM microcavity with large $Q$ factor not only provides resonances with narrow linewidth but also decreases the lasing threshold when doping 
with active materials due to the lengthy processes of the light-gain interactions. ${ }^{[28]}$ Experimentally, the $Q$ factor can be measured as: ${ }^{[15]}$

$$
Q=\frac{\lambda}{\Delta \lambda}
$$

where $\lambda$ is the resonant wavelength and $\Delta \lambda$ is the full width at half maximum (FWHM) of the resonance measured from the spectrum. Microcavities with $Q$ factors that are within the range of $10^{3}$ to $10^{6}$ are called high- $Q$-microcavities, while the ones with $Q$ factors larger than $10^{7}$ are considered to be ultra-high- $Q$-microcavities. ${ }^{[50]}$ The reported $Q$ factors of $>10^{11}$, which far outweigh the highest $Q$ factor of the FabryPerot microcavities $\left(\sim 10^{5}\right)$, indicate that the WGM microcavities are competitive and promising in microcavity studies. ${ }^{[56,57]}$

\subsubsection{Free spectral range}

Defined as the distance between two adjacent longitudinal modes, the free spectral range (FSR), or the mode spacing, can be expressed by: ${ }^{[6]}$

$$
2 n \pi R_{r}=m \lambda_{m}=(m-1) \lambda_{m-1}=(m-1)\left(\lambda_{m}+\Delta \lambda\right)
$$

where $\Delta \lambda$ is the FSR, $\lambda$ is the resonant wavelength, $m$ is the angular/longitudinal mode number, $n$ is the effective index, and $R_{r}$ is the effective radius of the resonant modes with respect to different radial mode numbers. Generally, the angular/longitudinal mode number $m$ far outweighs 1 in real cases ${ }^{[45]}$ and thus, Eq. (1.35) can be simplified as:

$$
\Delta \lambda=\frac{\lambda_{m}^{2}}{2 n \pi R_{r}} .
$$

For fundamental modes $(r=1)$, FSR is inversely proportional to the radius of the 
WGM microcavity, which has been proved both theoretically (Eq. (1.36)) and experimentally. ${ }^{[15]}$

\subsubsection{Mode volume}

The electric and magnetic field localization is referred as mode volume of a WGM microcavity. It is defined by: ${ }^{[6]}$

$$
V=\frac{\text { Total Energy }}{\text { Max(Energy Density })},
$$

where $V$ is the mode volume, the denominator is the maximum electromagnetic (EM) energy density, and the numerator is the EM energy stored in a particular mode.

WGM microcavities always provide small mode volumes, ${ }^{[6]}$ which is beneficial for the increase of both the spontaneous emission and stimulated emission rates. ${ }^{[58]}$ Meanwhile, as the lasing threshold drops with the decrease of the cavity mode volume, ${ }^{[58]}$ the WGM microcavities are promising candidates for low-threshold lasers.

\subsubsection{Lasing threshold}

Lasing threshold is described as the power needed to achieve the equilibrium of optical gain and optical loss within an active cavity. The lasing threshold is closely related to the $Q$ factor, the mode volume of the cavity, the corresponding materials, and the excitation schemes. Therefore, the determination of the lasing threshold of a particular microlaser is typically based on the point where the photon generation equals to the photon loss, which is named as the critical point. ${ }^{[59,60]}$ From the view of cavities, the WGM microcavities present high $Q$ factors, small mode volumes and 
enhanced light-matter interactions, which enable them to be one of the best choices to achieve low-threshold lasing with the lasing thresholds down to $\mu \mathrm{J}$ and $\mathrm{nJ}{ }^{[61,62]}$

\subsection{The advances of the whispering gallery mode microcavities and microlasers}

Engineering facile WGM microcavities with high quality for commercial and industrial applications has always been attractive in the research of microcavities. Thanks to the development of material science and manufacturing technologies, the fabrication methodology for WGM microcavities and microlasers evolved from the melting of glassy materials in early stages, to the more precisely controlled top-down and bottom-up processing of semiconductors, then to the flexible, tunable, and self-assembled polymer microcavities. In this section, the WGM microcavities are reviewed from the aspect of fabrications methodologies. Meanwhile, various geometries like the microspheres, hemispheres, microfibers, microdisks, and microtoroids are also discussed.

\subsubsection{Processing of the glassy candidates}

In the early stages, the studies of WGM microcavities were mainly based on microspheres, which are formed by the surface-tension-induced self-assembly of glassy powders or fiber tips after melting. ${ }^{[53]}$ Although the self-assembled glassy microcavities are ideally spherical due to the nature of surface energy minimization, their $Q$ factors may be suppressed because of the existence of the environmental 
contaminants during the drying process, which induces enlarged scattering loss. Therefore, chemical polishing is always applied to enhance the quality of the microspheres. ${ }^{[6]}$ The melting techniques vary from the crucible/furnace melting, ${ }^{[63]}$ laser melting, ${ }^{[64,65]}$ plasma torch melting by microwave, ${ }^{[66]}$ to electric arc melting. ${ }^{[67]}$ However, one of the disadvantages of the microcavities and microlasers fabricated by melting methodologies is the lack of control of cavity size. An advanced and controllable technique is to take advantage of the arm of a splicer, where a pre-softened glassy fiber was inserted. The splicer provides electric arcs to melt the fiber tip to further form a microsphere. By controlling the intensity and number of the electric arcs, the fiber-splicing method can control the size of the fabricated microspheres, with a minimum diameter of $40 \mu \mathrm{m} .{ }^{[68]}$

Microspheres can also be fabricated by the sol-gel approach, which is a chemical process that grows the spheres in solution from tiny precursors and then dehydrates the sol after synthesis. ${ }^{[69]}$ Besides, microbottle and microbubble microcavities were also well-explored in the early studies not only because of the similarity with the glassy microspheres in fabrication but also due to the better confinement of WGM modes in axial direction comparing with the microcylinders. ${ }^{[6]}$ The microbottles are normally fabricated by stretching or compressing the specific points of the glassy fibers after softening ${ }^{[70,71]}$ and the microbubbles are formed by the enlarged gas pressure within the heated microcapillaries. ${ }^{[72,73]}$ In general, due to the limitation of the equipment and techniques, the fabrication of the glassy WGM microcavities in early stages lacked precise control, which hindered the development of these 
candidates.

\subsubsection{Top-down and bottom-up of semiconductor processing}

As mentioned in section 1.1, WGM microcavities and microlasers have been widely applied in integrated photonic circuits, which require compact device distribution and small device volume. ${ }^{[53]}$ Therefore, semiconductor-based two-dimensional (2D) WGM configurations like the microdisks, microrings and microracetracks have been well-studied. ${ }^{[74-76]}$ Comparing with microspheres, microbottles and microbubbles, the planar WGM geometries are not only more stable and space-efficient for on-chip integration, but also better in the suppression of higher order modes, especially in the azimuthal direction. ${ }^{[6]}$ In addition, the semiconductors normally provide relatively high refractive indexes so the diameters of the WGM microcavities and microlasers based on semiconductor materials can be reduced to the level of several micrometers or even nanometers. ${ }^{[77,78]}$

In general, the processing of semiconductor materials can be divided into the top-down and bottom-up approaches. For the former method, the epitaxially grown wafers are etched ${ }^{[78]}$ with techniques such as electron-beam lithography (EBL), dry/wet etching, focused ion beam (FIB) etching, deep ultraviolet lithography and nanoimprinting in order to control the size and position of the WGM microcavities and microlasers. ${ }^{[50,53]}$ One example is the fabrication of a microtoroid cavity (Fig. 1.9). Microtoroids are the evolution of semiconductor-based WGM microcavities because they break the limitation of $Q$ factors brought by surface roughness during 
the fabrication of conventional microdisks and microrings. ${ }^{[79,80]}$ As shown in Fig. 1.9, the silica layer is etched via dry etching technique after deposition on the substrate. Then wet etching is used to shape the silicon wafer as a pedestal. At this point, a microdisk cavity is successfully fabricated. However, in order to further increase the $Q$ factor, $\mathrm{CO}_{2}$ laser illumination is implemented to melt the microdisk and self-assemble the microtoroid, exploiting surface tension and the difference of heat conductivities between silica and silicon. ${ }^{[6]}$ Comparing with the $Q$ factors of microdisks $\left(10^{4}\right),{ }^{[50]}$ the microtoroids can reach a $Q$ factor of $10^{8}$ with the diameter around $100 \mu \mathrm{m}$ and thickness around $10 \mu \mathrm{m}$, which makes microtoroid cavities desirable elements in photonic circuits.

\section{Deposition}

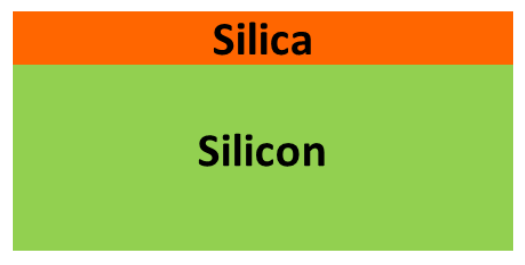

4. $\mathrm{CO}_{2}$ laser illumination

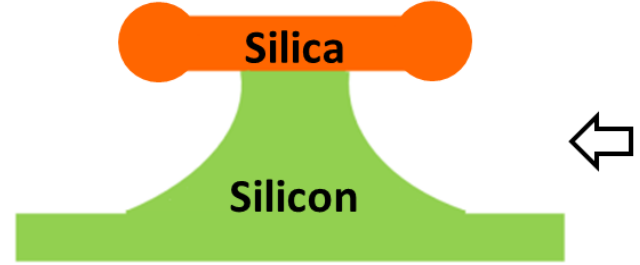

\section{Dry etching of Silica}

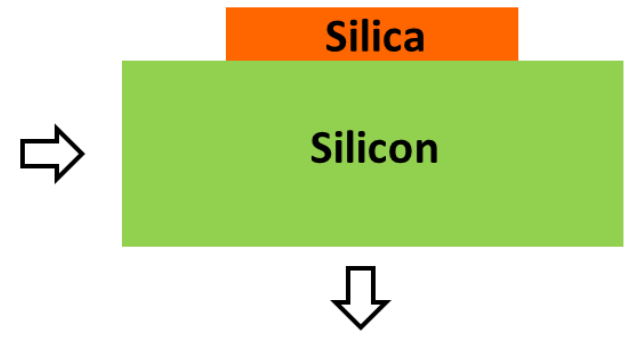

3. Wet etching of Silicon

Silica

\section{Silicon}

Figure 1.9. The top-down approach of a microtoroid cavity with high $Q$ factor. ${ }^{[6]}$

Nanosphere lithography, or NSL, is a significant technique among top-down approaches to realize high-throughput fabrication that the conventional methods like EBL and FIB etching cannot achieve. ${ }^{[78,81]}$ In NSL, the self-assembled nanosphere arrays are initially spin coated on the wafer and then removed with the help of 
techniques such as inductively coupled plasma (ICP) etching, leaving the periodic patterns. ${ }^{[82-84]}$ After appropriate post-processing treatments like electron-beam evaporation, nano-arrays of rings hemiellipses, or even pillars that sustain WGM modes can be obtained. ${ }^{[83-85]}$ In addition, dimensional tweaking, or sphere-shrinking, is also a commonly applied procedure to increase the flexibility of NSL. By adjusting the size of the spin coated nanospheres via reactive ion etching (RIE) before removal, the parameters of the resulted nano-arrays can be controlled. ${ }^{[86]}$

Although the top-down techniques can control the fabrication of the microcavities with a resolution down to the level of nanometer, ${ }^{[6]}$ the drawbacks of these methods like sophisticated equipment, low-throughput, limited materials due to the requirement of lattice matching in epitaxial growth, and the surface roughness induced by imperfect lithography or etching cannot be ignored. ${ }^{[6,78]}$ In contrast, the bottom-up approaches, which are mainly based on the crystallization of semiconductors, can provide higher throughput and smoother cavity surface. ${ }^{[78]}$ Therefore, they are important in semiconductor processing. Meanwhile, the growth of the semiconductor crystals can be well-controlled in size and geometry by adjusting the temperature, pressure, humidity, as well as the operation time. ${ }^{[6]}$ Fig. 1.10 shows an example of the hexagonal $\mathrm{ZnO}$ microdisk synthesis via the bottom-up approach. ${ }^{[87]}$ In process 1 , the $\mathrm{ZnO}$ nanowires were grown on sapphire substrates and then, with the increase of the growth time, the nanowires became thicker and thin films were formed (process 2). The hexagonal $\mathrm{ZnO}$ microdisks were synthesized on the top of the thin films with further prolonged growth time and the size of the microdisks could be 
controlled (process 3-5). Furthermore, low-threshold ultraviolet WGM lasing was observed from the $\mathrm{ZnO}$ microdisks and the peaks showed sensitive responses to the perturbation of the environmental refractive index.
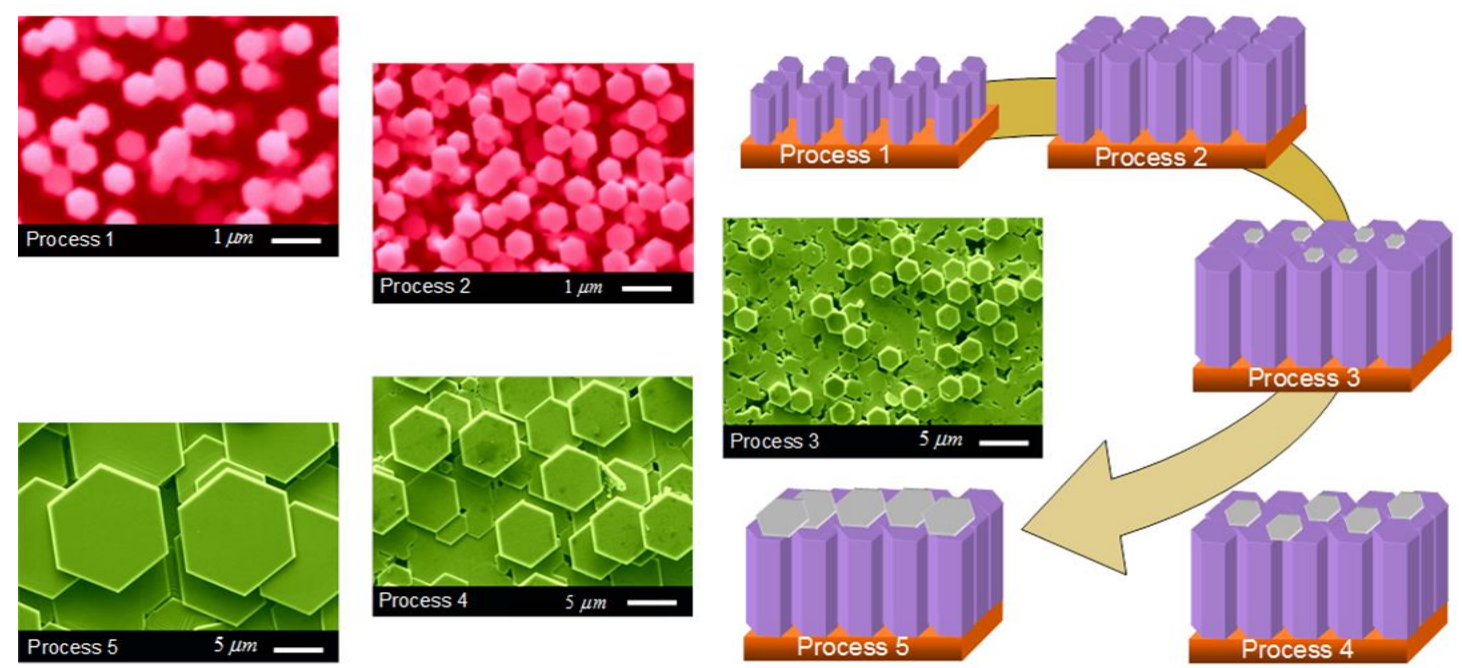

Figure 1.10. Bottom-up approach of the $\mathrm{ZnO}$ microdisk lasers. ${ }^{[87]}$

The core-shell structure, or coating gain medium outside conventional passive microcavities, is attractive not only because of the high-quality optical performances but also due to the possibility of solving the problem of incompatibility between the gain and the cavity material. ${ }^{[88]}$ Up to now, mature techniques like the electron beam evaporation, low-pressure chemical vapor deposition (LPCVD), dip-coating, as well as sol-gel process have been widely applied in the fabrication of microcavities and microlasers with core-shell structures. ${ }^{[53,69,88-91]}$ In general, the core-shell structures can be divided into two types according to the relative values of the refractive indexes of the core and the shell. Although the influences of environmental perturbations can be minimized, having a shell with a refractive index which is lower than the core can suppress the coupling efficiency of the WGM modes with waveguides due to the 
reflections at the boundaries of the shell and the core. ${ }^{[92]}$ Meanwhile, the evanescent waves are weak in such structures as they are dramatically blocked in the shell. ${ }^{[92]}$ In contrast, the second type of core-shell structure, in which the refractive index of the shell is larger than the core, exhibits relatively low $Q$ factor due to the absorption in the shell and the scattering loss at the core-shell boundary. ${ }^{[53]}$

\subsubsection{Soft-material processing}

Although the semiconductor processing techniques are mature and controllable, the sophisticated equipment and complex operation parameters are obstacles for cost-efficient commercialization. In addition, modern applications such as sensors require the microcavities and microlasers to be flexible, tunable, and facile in doping and wavelength manipulation, which are hard to be achieved in semiconductors due to the rigid nature of the materials. ${ }^{[15]}$ Therefore, the soft-approached WGM microcavities and microlasers have attracted enormous attention because of their advantages like high flexibility and tunability, easy processing, and cost economization. $^{[6]}$

The pioneer studies of soft-approached WGM microcavities and microlasers in the 1980s are based on microdroplets, which are self-assembled due to the surface tension effect of the liquid materials. ${ }^{[93-95]}$ However, the microdroplets experience free-falling motion after the fabrication via the vibrating orifice droplet generator, which decreases the lifetime of the microdroplets and enhances the difficulties in spectrum capturing. ${ }^{[93]}$ In order to address these problems, techniques like 
microdroplet-levitation via electrodynamic traps, ${ }^{[96]}$ ultraviolet solidification after fabrication, ${ }^{[97]}$ as well as free-standing microdroplets on super-hydrophobic layers ${ }^{[98]}$ were proposed. Moreover, the WGM microdroplets are also applied in the microfluidic systems to further broaden the applications in biology and chemistry. ${ }^{[99-101]}$ Nevertheless, the spherical geometry of the microdroplets has the intrinsic drawback of being unstable (or needs complex setups to stabilize the droplets), which limits the applications of the microdroplets in photonic circuits due to the difficulties in large-scale integration.

The emergence of the polymer-based WGM microcavities and microlasers has brought the soft-approached candidates to a new level due to the merits of facile fabrication, outstanding optical performances, mechanical flexibility, long lifetime, as well as the possibility of integration. ${ }^{[6]}$ One example is the hemispherical lasers. ${ }^{[28]}$ As shown in Fig. 1.11 (a), in this example the microcylinder was first coated with hydrophobic solution and then spread with dye-doped polymer materials. Then the line-shaped polymers would break into microdroplets and hang discretely on the microcylinder due to the effect of hydrophobicity and gravity. After gentle touching with the distributed Bragg reflector (DBR) substrate, which was also spin coated with a layer of hydrophobic materials on the top, the discrete microdroplet array on the microcylinder was transferred to the surface of the DBR substrate and self-assembled to be hemispheres. The DBR substrate not only acted as a sample holder for the hemispheres but also helped to confine the light in the vertical direction to enhance the light-matter interaction. These circular hemispheres with various diameters (Fig. 
1.11 (b)) can sustain WGM resonances with $Q$ factors as high as 8000 , which is higher than the reported microdroplets. ${ }^{[46]}$ Meanwhile, single mode lasing and sensitive responses to the changes of the environmental refractive index have also been observed from the hemispherical configuration. ${ }^{[10,47]}$ In addition, active gain materials can also be deposited outside the hemispheres, which is similar to the core-shell structure of the semiconductors. It is reported that high-quality WGM lasing was obtained from passive polymer hemispheres with an outer layer of dip-coated colloidal quantum dots (CQDs). ${ }^{[20]}$ This coating method can be used with a variety of gain materials that are not compatible with polymers after mixing due to the complex ligand-exchange process which induces inhomogeneous dispersion of the gain inside the cavity. Therefore, diverse colored lasing can be achieved via this facile and cost-efficient process, which is significant for laser illumination and display applications.

(a)

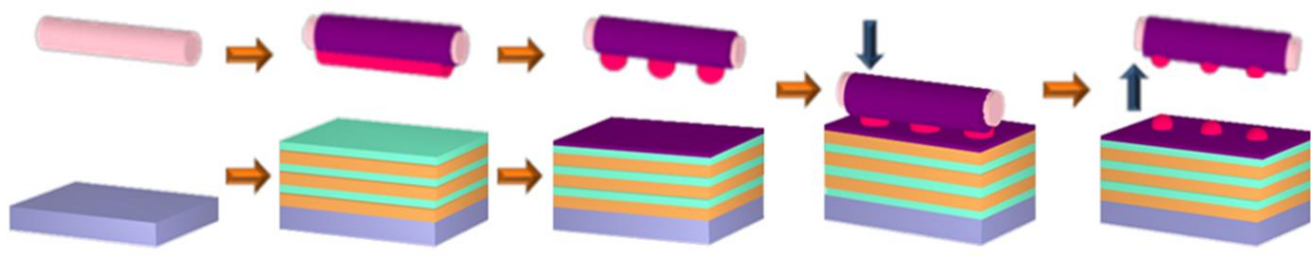

(b)
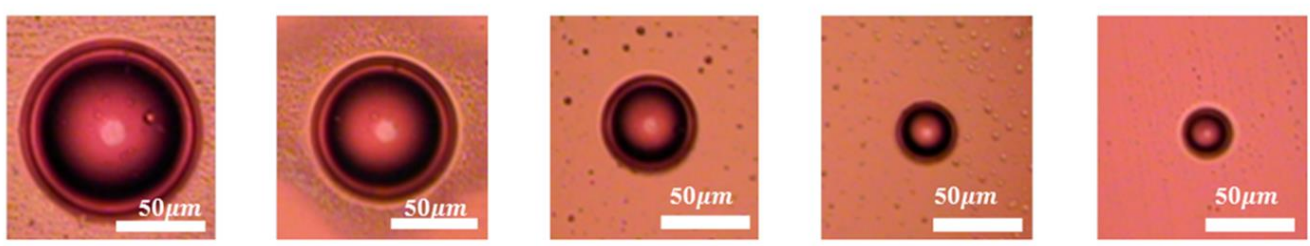

Figure 1.11. The soft-approached hemispherical microlasers.

(a) Schematic of the fabrication processes of the hemispheres. (b) Optical images of the hemispheres with different diameters. ${ }^{[28]}$

The realization of the WGM microfiber laser is a breakthrough in fiber optics as 
previously the microfibers are mostly used as waveguides ${ }^{[102]}$ and the limited studies of the lasing performances are based on the Fabry-Perot mechanism. ${ }^{[103-105]}$ The WGM microfiber laser was fabricated via a simple technique called direct drawing. ${ }^{[48]}$ As shown in Fig. 1.12 (a), after dipping into the viscous cavity materials, the metal tip was lifted up and then the microfibers were drawn on the substrate. The optical images shown in Fig. 1.12 (b) illustrate that uniform microfibers with different diameters can be self-assembled via the direct drawing technique. Moreover, high-quality WGM lasing ( $Q$ factor $\sim 8200)$ from the circular cross-section of the microfibers were observed and verified. By substituting the solid substrate to polydimethylsiloxane (PDMS) elastomer, the direct-drawn microfiber lasers can be buried and better protected. ${ }^{[45]}$ Also, the PDMS-microfiber system with improved flexibility can be utilized as a type of wavelength modulator due to the refractive index variation brought by the elastic PDMS matrix under physical deformation. In addition, the outstanding optical performances of the single and coupled microfiber lasers enable this promising configuration to be exploited as sensitive refractive index sensors. ${ }^{[32,48]}$ It is important to note that the polymer microfiber lasers not only show prominent characteristics in lasing, but also keep the inherent properties as optical waveguides. Therefore, they are good choices for the ultra-compact photonic circuits. $^{[106,107]}$ 


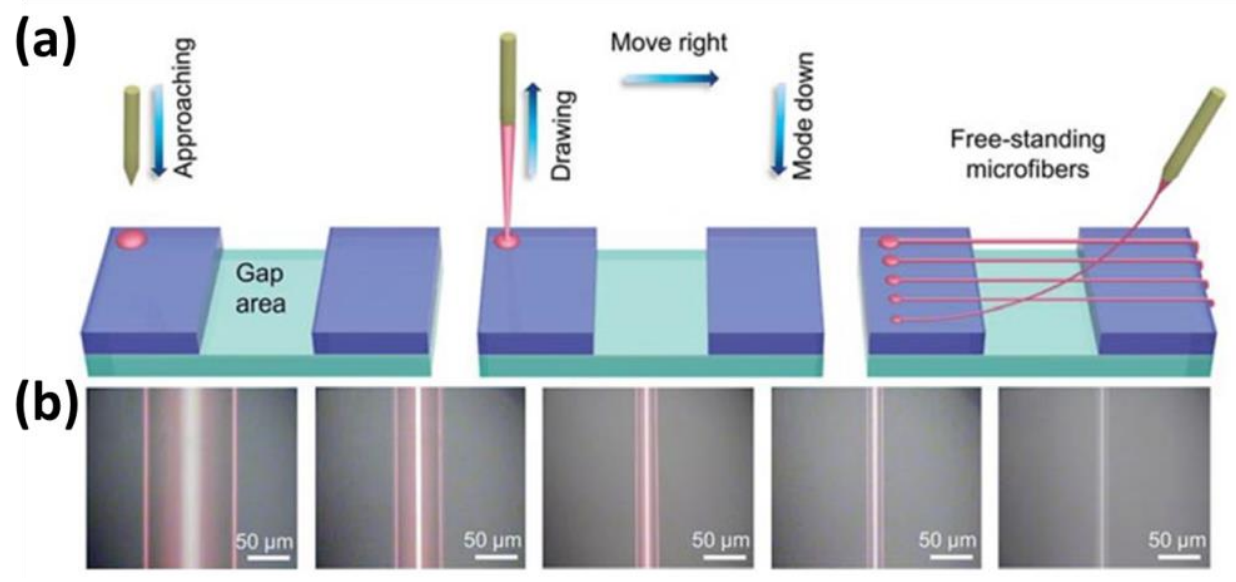

Figure 1.12. The polymer microfiber lasers via direct drawing technique. (a) Schematic of the direct drawing technique for the fabrication of microfiber lasers. (b) Optical images of the microfibers with different diameters. ${ }^{[48]}$

In summary, cavity-surrounding materials of the soft-approached microcavities and microlasers evolved from the liquid-air combination in the 1980s, to liquid-liquid, liquid-polymer, and then to the recently reported polymer-polymer/air. ${ }^{[6]}$ Meanwhile, the fabrication techniques have become much more facile and cost-efficient and the WGM microcavities and microlasers have shown better optical performances and more stable structures. Although the robustness of the soft-approached candidates is sacrificed comparing with the conventional glassy and semiconductor ones, the characteristics of flexible doping, tunable mechanical properties, as well as self-assemble processing brought by the intrinsic nature of the soft materials have made up the deficiencies of the conventional solid-state architectures and extremely broadened the applications of WGM microcavities and microlasers.

\subsection{Wavelength tuning of the whispering gallery modes}

Tuning the resonant wavelengths in the cavity is one of the significant branches 
in WGM microcavity and microlaser studies. Conventional solid WGM candidates based on semiconductors and glassy materials are hard to tune due to the rigid nature. ${ }^{[6]}$ In contrast, soft-approached WGM microcavities and microlasers hold the advantages of being flexible in doping, simple in fabrication and mechanically elastic, which enable them to be facile in wavelength tuning. Therefore, most of the reported works in this field are based on soft materials like polymers and liquid crystals with various tuning mechanisms.

\subsubsection{Gain material modification}

Generally, the resonant modes in a WGM microcavity are sensitive to three factors: the gain medium, the effective index and the size of the cavity. ${ }^{[6]}$ Therefore, the reported tunable microlasers are mostly based on the manipulation of these three conditions. Indeed, modifying the gain medium in a single WGM microlaser is still technically difficult after the microcavity is fabricated although various scientists have studied the properties of the gain materials for many years. ${ }^{[108-111]}$ Thus, the demonstrations of wavelength tuning by tailoring gain-medium-variation basically rely on indirect approaches like the multi-cavity-cooperation exemplified by the work introduced in Chapter 3. Another example is given by microdroplets, which are fabricated by the T-junction that can alternatively generate samples doped with different organic dyes, floating in a microfluidic channel (Fig. 1.13 (a)). ${ }^{[12]}$ As the excitation and collection system are fixed and the microdroplets move quickly in the microfluidic channel, the recorded spectra show fast-switching behavior in two 
wavelength ranges (Fig. 1.13 (b)). Meanwhile, the tuning or switching speed of the two wavelengths can be well-controlled by the T-junctions and the pressure of the microfluidic channel.

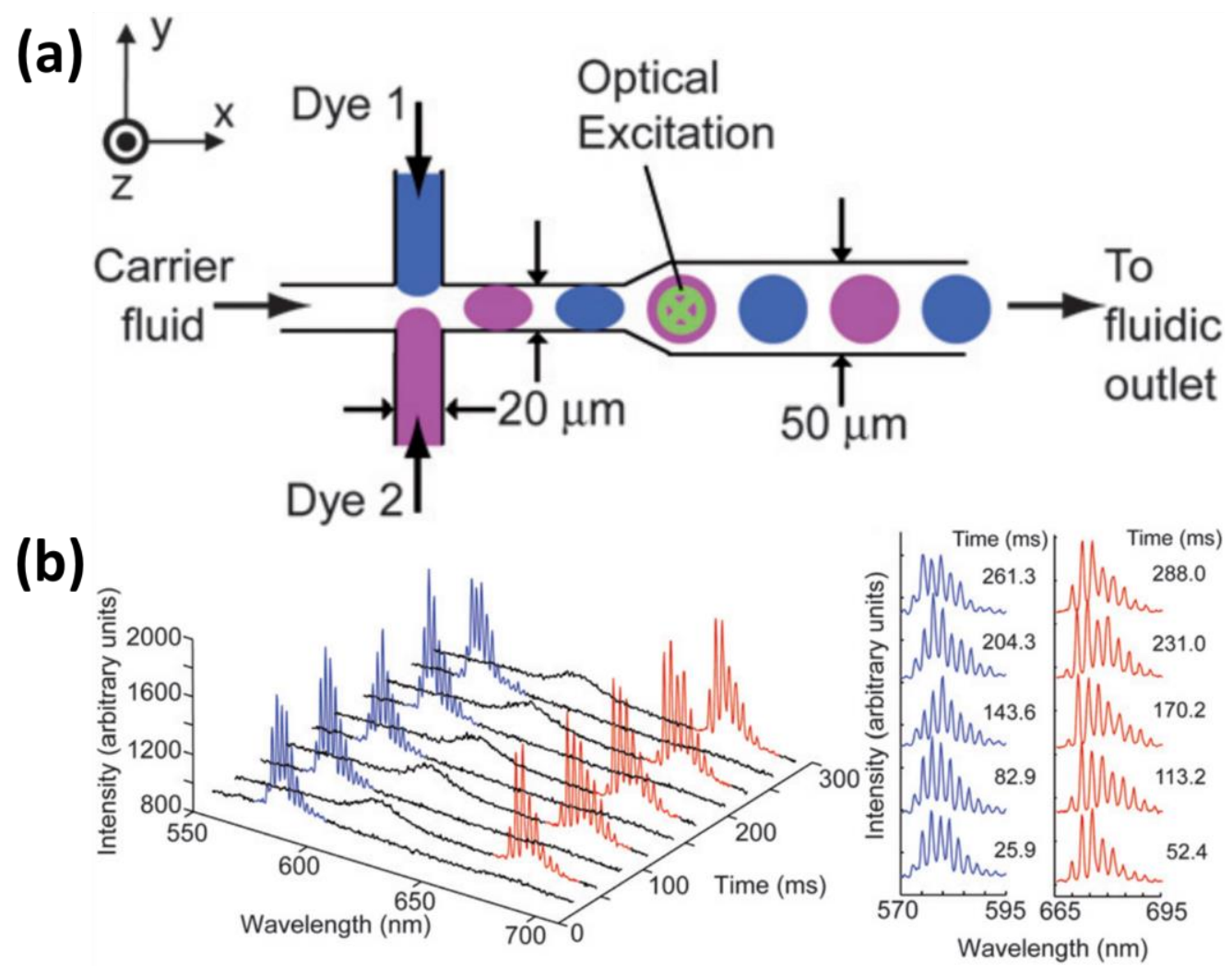

Figure 1.13. Realization of wavelength tuning by multi-cavity-cooperation.

(a) The structure of the T-junction and the microfluidic channel. (b) The fast-switched lasing spectrum. ${ }^{[112]}$

\subsubsection{Effective index variation}

The modification of effective index normally utilizes the index change of the cavity materials or surrounding materials under external forces or fields. For example, the alignment of the nematic liquid crystals (NLCs) is different with and without external electric field, which induces different dielectric constants for the TM modes and, therefore, shifts the spectrum. ${ }^{[113]} \mathrm{R}$. Chen et al. demonstrated the shift of the lasing peaks of the microfiber lasers via bending the PDMS matrix to "dilute" or 
"compress" the polymer, which resulted in the index-variation of the surrounding material. ${ }^{[45]}$ In addition, the thermo-optic coefficient of a cavity material, which is determined by the variations of thermal-energy-induced material absorption and thermal-expansion-induced material density, can also be utilized to manipulate the index. ${ }^{[53,114-116]}$ For example, the nanocrystalline silicon coating layer conducts the excitation energy to the inner silica microsphere by absorption and induces the refractive change of the microresonator, which results in a fast thermooptical switch. ${ }^{[117]}$

\subsubsection{Cavity size tuning}

As the sizes or the shapes of the WGM microresonators are easily affected by the environment and the intrinsic material properties, especially for the soft-approached ones, there are various methods for cavity shape deformation. By taking advantage of the controllable environmental humidity in a chamber, A. Kiraz et al. successfully tuned the size of the microdroplets via evaporation and condensation and realized the shift of the lasing peaks. ${ }^{[98]}$ S. K. Tang et al. exploited the solubility of benzyl alcohol in water to achieve the continuously decreasing microdroplets in a microfluidic channel. ${ }^{[46]}$ The lasing envelope shifted around $80 \mathrm{~nm}$, which is large compared with other works. Furthermore, as shown in Chapter 4, irregular WGM cavity shape caused by external perturbations can also lead to the shift of the modes. Another example is the directly drawn polymer microspheres in PDMS, which can be deformed via external stretching forces and results in the redshift of the lasing modes. ${ }^{[118]}$ 


\subsection{Motivation and objectives}

As introduced in section 1.4, the development of WGM microcavities and microlasers is based on the requirements of practical applications. Therefore, engineering novel, facile and multifunctional WGM microcavities and microlasers for practical applications has always been attractive for researchers. The glassy microspheres in early studies mainly contributed to the fundamental studies of WGMs while the top-down and bottom-up approaches of semiconductors addressed practical applications and on-chip integration in photonic circuits. However, modern applications not only require the microcavities to be robust and integratable but also flexible, highly tunable and reversible. ${ }^{[15]}$ Although the reported polymer WGM microcavities and microlasers have the advantages of flexible doping, tunable mechanical properties, as well as self-assembled fabrication, which can solve some of the problems met by the solid-state counterparts, there also exist challenges like the coarse resolution brought by the manual operation during fabrication, restricted field of applications, as well as limited configurations. Thus, the motivation and objectives of the works in this thesis are to address the challenges of the soft-approached WGM microcavities and microlasers and achieve the goals of controllable fabrication with high resolution, application broadening, and novel WGM microlaser design.

In Chapter 2, the experimental setups and systems that are relevant to the works in this thesis are introduced. Chapter 3 proposes a precise fabrication technique for polymer hemispherical microlasers. In addition, multicolor lasing is achieved by deploying two types of gain materials with different ratios, which can be potentially 
applied in laser displays. Microfiber laser arrays are fabricated with controlled position and diameter via the advanced spiral drawing technique and further applied as strain and force sensors in Chapter 4. Chapter 5 introduces a novel quasi-disk floating microlaser, which is tunable, reconfigurable and can be utilized as a sensitive sensor for water-soluble organic compounds. Last but not least, the conclusions, as well as the perspectives of the WGM microcavities and microlasers, are discussed in Chapter 6. 


\section{Experimental Methodologies}

\subsection{GIX ${ }^{\mathrm{TM}}$ Microplotter $^{\mathrm{TM}}$ II}

The GIX ${ }^{\mathrm{TM}}$ Microplotter ${ }^{\mathrm{TM}} \mathrm{II}$ is a commercially available system used for pattern design and high-throughput manufacturing. With the advantages of simple operation, high resolution, multiple fabrication approaches, as well as relatively low cost comparing with other commercial systems in semiconductor processing, the microplotter is shown to be competitive in the design and printing of microstructures with soft materials. ${ }^{[119-124]}$

The microplotter is mainly composed of five parts, the X-Y-Z stage, the hollow microdispenser, the ultrasonic vibrator, the camera and the computer-based control system (shown in Fig. 2.1 (a)). The $\mathrm{X}-\mathrm{Y}-\mathrm{Z}$ stage is a motor-controlled three-dimensional platform with the stepping resolution down to $1 \mu \mathrm{m}$. The hollow microdispenser is made of glassy materials and the tip diameters range from $10 \mu \mathrm{m}$ to $30 \mu \mathrm{m}$. Attached with the microdispenser, the ultrasonic vibrator is implemented to clean the dispenser after fabrication by oscillating the soft materials out with the help of other solvents like ethanol and acetone. The hollow microdispenser and the ultrasonic vibrator are installed on the $\mathrm{X}-\mathrm{Y}-\mathrm{Z}$ stage and can be removed and changed according to different requirements during fabrication. The camera is used to provide the optical images of the microdispenser to the control system during fabrication and the control system is responsible for the design of patterns, control of the $\mathrm{X}-\mathrm{Y}-\mathrm{Z}$ stage and the ultrasonic vibrator, as well as monitoring the fabrication process. 


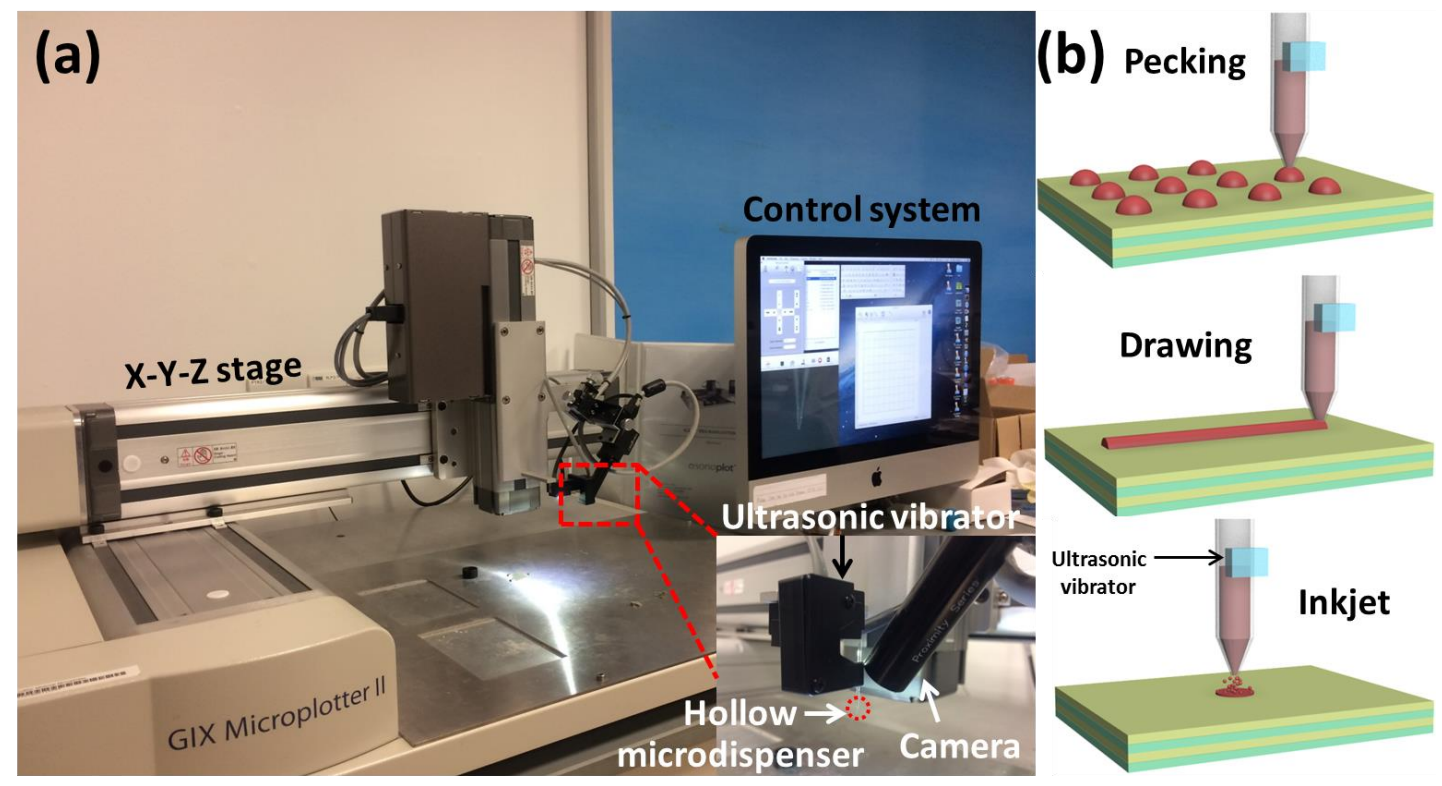

Figure 2.1. The GIX ${ }^{T M}$ Microplotter ${ }^{T M}$ II microplotter.

(a) The image of the Microplotter. The inset shows the enlarged part of the microdispenser. (b) The schematic of the fabrication approaches of the microplotter.

Basically, the first step in microplotter processing is to immerse the microdispenser into the soft materials. Due to the capillary effect, the liquids will be inhaled into the dispenser and arched liquid surface will be formed at the tip after the microdispenser is lifted because of the gravity and surface tension. Then, as illustrated in Fig. 2.1 (b), the fabrication stages of the microplotter are differentiated into pecking, drawing and inkjet. When touching/pecking the substrate gently from the vertical direction, a small volume of the soft materials is left on the surface and self-assembled to be various geometries according to the properties of the materials. ${ }^{[21,}$ ${ }^{119]}$ After surface calibration, the microdispenser can move smoothly on the surface of the substrate and draw the designed pattern with lines. Inkjet deposition is obtained with the help of the ultrasonic vibrator as the tip of the microdispenser does not touch the surface of the substrate. The soft materials are forced to be ejected from the 
microdispenser by oscillation and cover the desired area to achieve coating in the ultra-small area. The inkjet technique depends critically on the lifting height of the microdispenser and the oscillation strength of the ultrasonic vibrator.

\subsection{Ultrasonic vibrator}

The ultrasonic vibrator, which utilizes the cavitation bubbles produced by ultrasonic waves to agitate the liquid, is widely employed in solution mixing and contaminant cleaning. ${ }^{[125]}$ The cavitation bubbles are created by a rate of millions of units per second and break quickly to generate large agitation forces, which can not only mix the solution homogeneously but also remove the surface contaminants attached to the objects that are immersed in the liquid. The cavitation bubbles do not damage the immersed objects and can reach anywhere as long as the liquid exists. Fig. 2.2 shows the image of a typical ultrasonic vibrator, which is larger and more powerful than the one used in the microplotter system. In addition, the thermal controller in this ultrasonic vibrator enables it to heat the liquid during working in order to get better results with the help of enhanced molecular movements. 


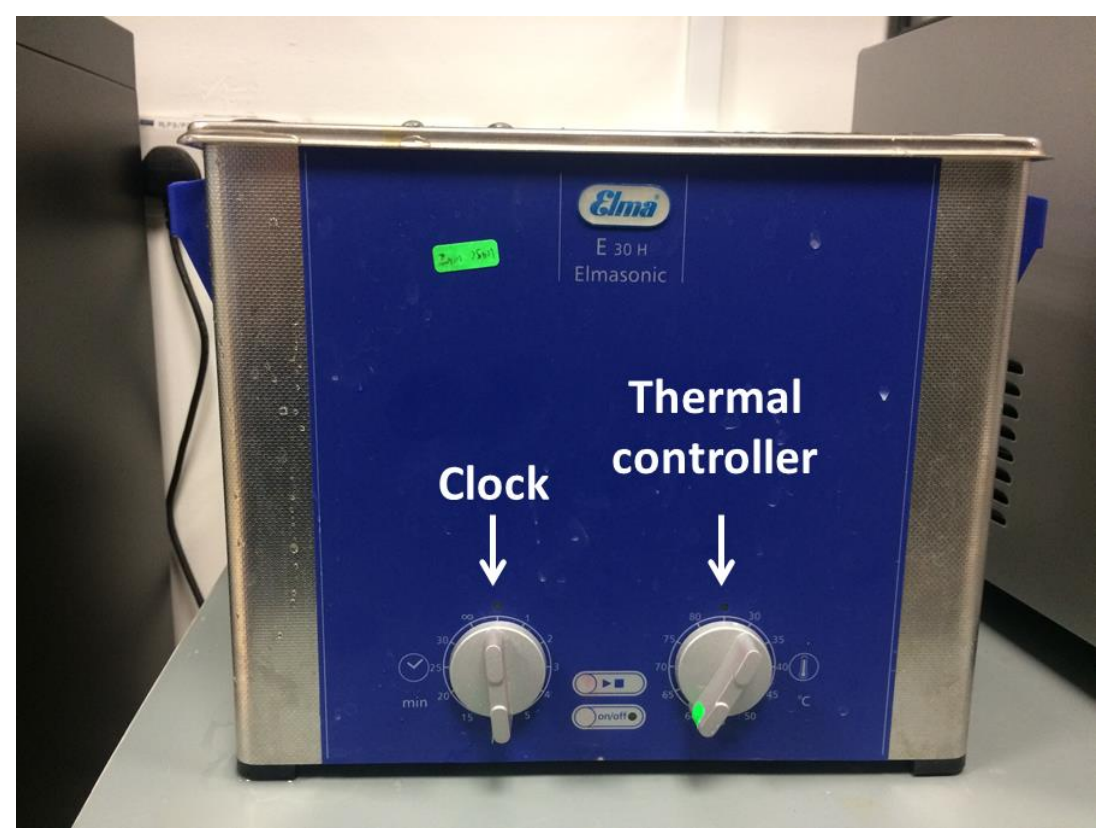

Figure 2.2. The ultrasonic vibrator.

\subsection{Spin coater}

The utilization of a spin coater is to uniformly spread a liquid material on the surface of the substrate or other materials. As demonstrated in Fig. 2.3, there is a sample holder in the middle of the chamber, which is connected with a vacuum pump and can hold the sample tightly during its high-speed rotation. After dropping several drops of the target liquid material on the fixed substrate, the chamber is closed and the sample holder rotates with the rotating speed as high as 6000 revolutions per minute (RPM), which results in a thin film of the target material on the substrate. The acceleration, rotating speed as well as the viscosity of the target material are important parameters to control the thickness and homogeneity of the spin-coated thin film. 


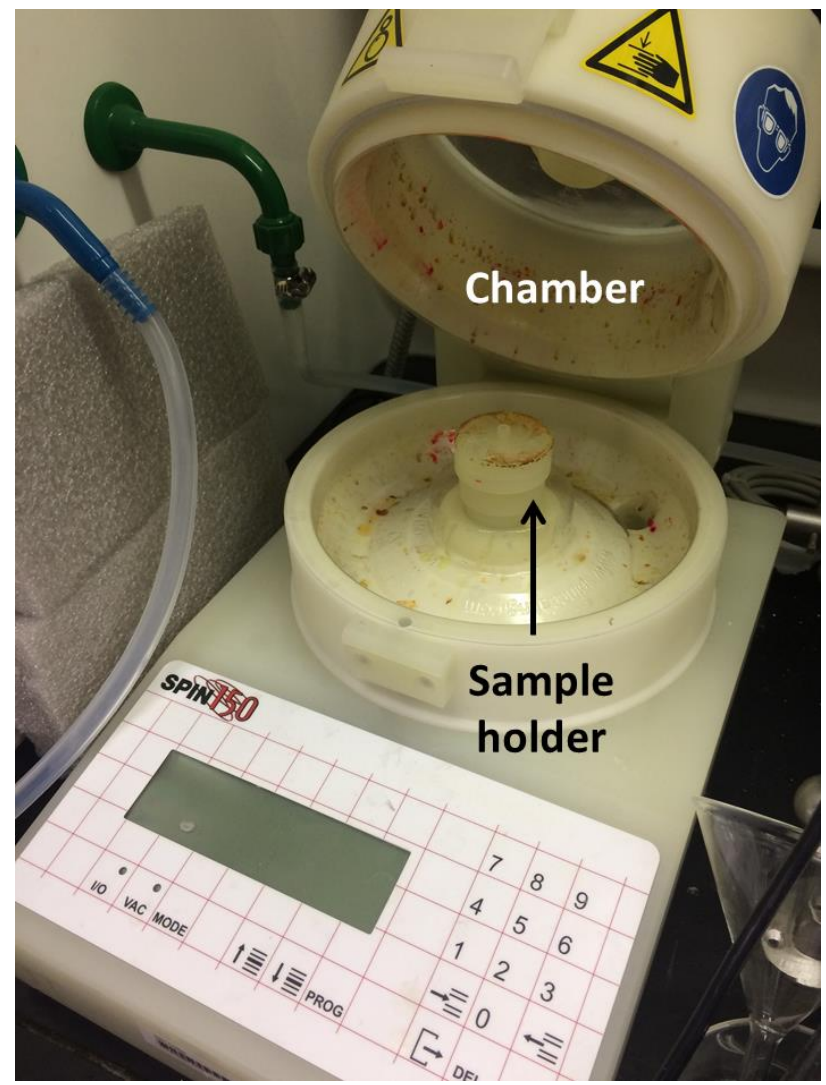

Figure 2.3. The spin coater.

\subsection{Scanning electron microscope}

The scanning electron microscope (SEM) is used to obtain the morphologies of the samples in the level of nanometer. Generally, the working principle of the SEM is to scan the surface of the sample with focused electron beams and then collect and analyze the various signals generated by the electron-atom interactions. The detected signals, which contain the information of topography and composition of the sample, normally include cathodoluminescence, X-rays, secondary and backscattered electrons. ${ }^{[126,127]}$ The SEM can provide a resolution as low as $1 \mathrm{~nm}$ and with the development of the SEM technologies, samples can be measured in various pressure, temperature, and humidity. ${ }^{[128]}$ Although the soft microlasers presented in this thesis are all in micrometer scale and can be clearly seen via the optical microscope, 
information like the geometry of the cross-sections of the microfibers still require the use of an SEM. The employed SEM model in this thesis is JEOL-6700F field effect scanning electron microscope (FESEM, shown in Fig. 2.4) and the soft samples are coated with a thin layer of gold in order to increase the conductivity and avoid the accumulation of electrons during measurement.

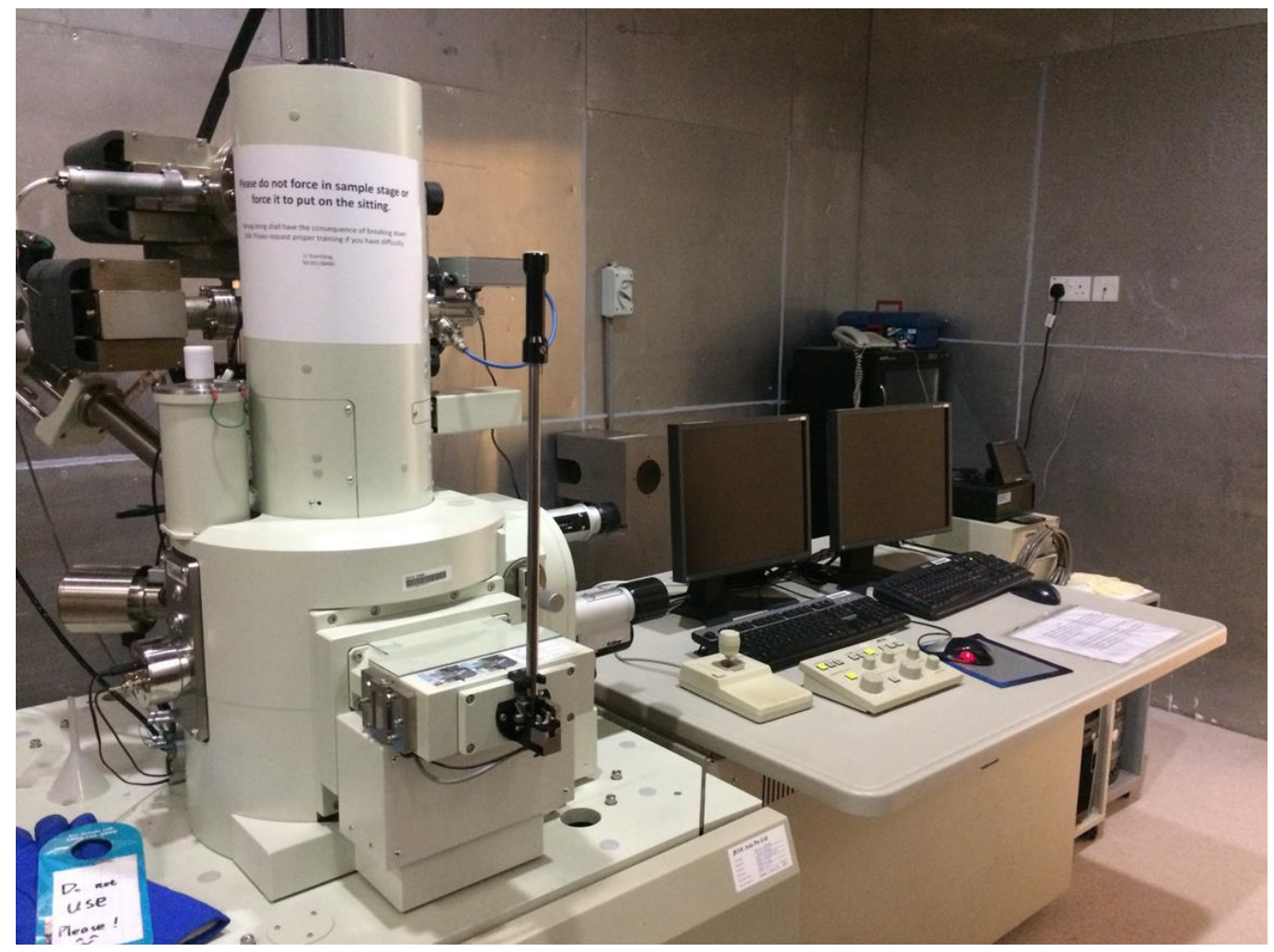

Figure 2.4. The JEOL-6700F field effect scanning electron microscope.

\subsection{Micro-photoluminescence system}

The micro-photoluminescence ( $\mu$-PL) system is used to investigate the optical performance of the samples. As shown in the schematic diagram of the $\mu$-PL system (Fig. 2.5), the excitation light from the Q-switched Nd: YAG laser with the wavelength of $532 \mathrm{~nm}$, pulse width of $1 \mathrm{~ns}$, and the repetition rate of $60 \mathrm{~Hz}$ is guided to the sample, which is fixed on a controllable $\mathrm{X}-\mathrm{Y}-\mathrm{Z}$ stage, via several mirrors and 
lens. Set directly above the sample, the objective $(50 \mathrm{X}$, numerical aperture $=0.42)$ is utilized to collect the emitted signals and provide optical images of the sample to the computer under the illumination with a halogen lamp. The collected signals are transmitted through a low-loss optical fiber and then recorded by a silicon charge-coupled device (CCD, noise level: 6 e-RMS at $100 \mathrm{kHz}$ ) which is linked to a monochromator. The spectral resolution is less than $1 \mathrm{~nm}$ and a long-pass filter is inserted in the optical path to prevent the damage of the CCD by the high-intensity excitation light.

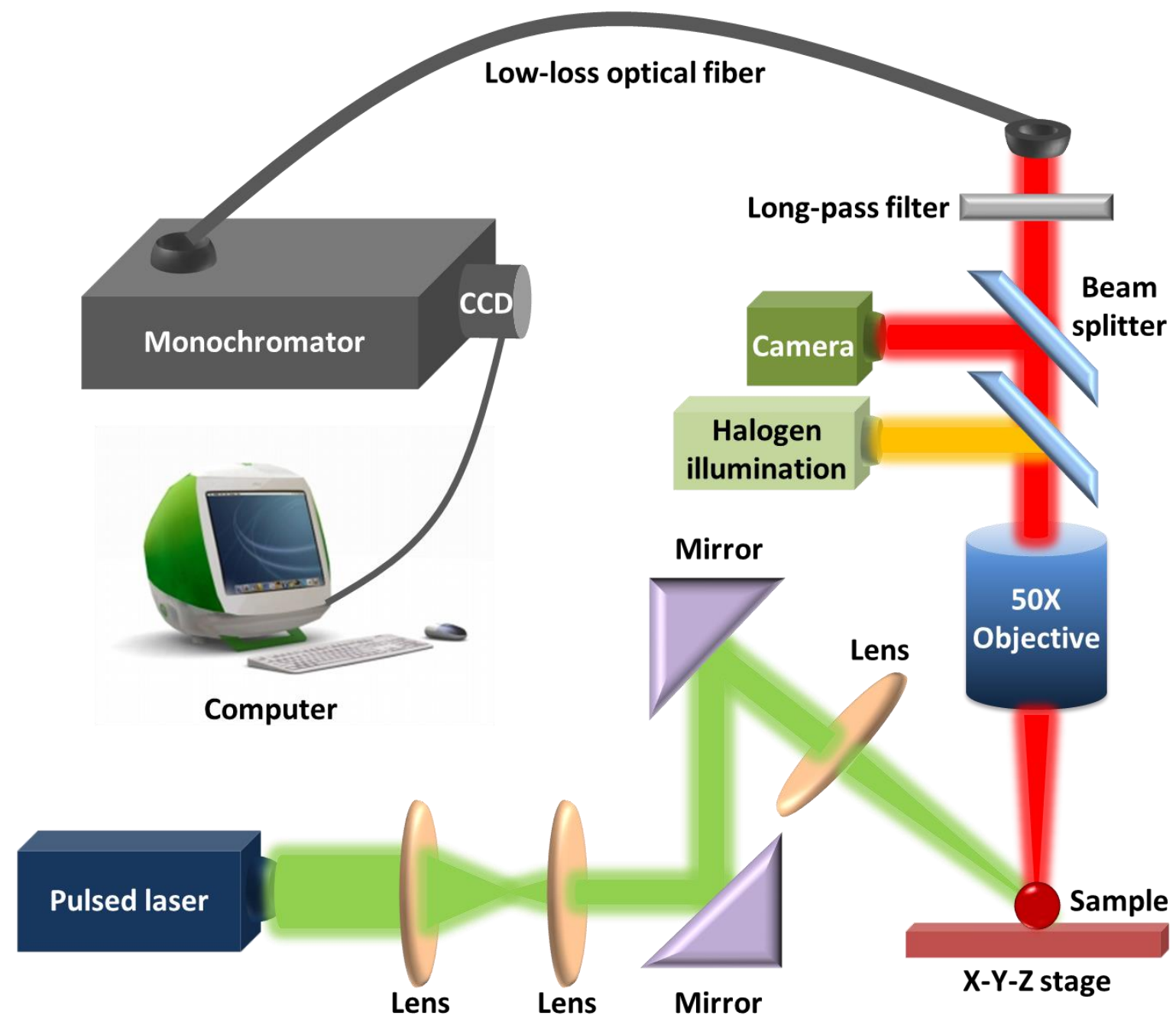

Figure 2.5. Schematic of the micro-photoluminescence system. 


\subsection{Time-resolved photoluminescence measurement}

Time-resolved photoluminescence (TRPL) measurements are used for the study of the dynamic processes of photoluminescence. ${ }^{[129]}$ A streak camera from Optronis, which can measure the wavelengths and lifetimes of the emission, is exploited in the work of this thesis. The streak camera utilizes the deflection of electrons (excited by the input optical pulses) under the high voltage of the synchronized sweep electrodes to resolve the input pulses spatially and temporally. ${ }^{[130]}$ The TRPL measurement is a useful method in spectroscopy, especially in the investigation of the Förster resonance energy transfer (FRET), which is a nonradiative energy transfer process between the donor and acceptor chromophores which are within the Förster distance. ${ }^{[131]}$ Detailed description and analysis of the TRPL spectrum and FRET can be found in section 3.4. 


\section{Printable and Multicolor Whispering Gallery Mode Hemispherical Lasers}

\subsection{Introduction and motivation}

As introduced in Chapter 1.4, the soft-approached WGM microcavities and microlasers have attracted enormous research interests due to their intrinsic flexibility in shape and doping, facile, simple and cost-effective fabrication processes. ${ }^{[6]}$ Among all the soft-approached candidates, the hemispheres, which are self-assembled and exhibit outstanding optical performances, are promising configurations for the applications in the fields of laser, sensor, optical communication, and on-chip integration. ${ }^{[10,28,47]}$ However, although the hemispheres can have diameters range from several micrometers to hundreds of micrometers, typically they are randomly formed by gently touching the microcylinder with the substrate or automatically breaking the microfibers. ${ }^{[28,47]}$ The lack of control in both position and size of the hemispheres during fabrication greatly hinders the development of this device and needs to be improved.

On the other hand, multicolor emission, especially multicolor lasing is attractive because of the tremendous potential in illumination and display. Compared with the commercialized light-emitting diodes (LEDs), laser illumination and display could overcome the efficiency droop without affecting the color rendering. ${ }^{[132,133]} \mathrm{Up}$ to now, scientists tried various methods to realize multicolor lasing or even white-light lasing. For example, multicolor nanowire lasing was achieved via a tapered 
microfiber with the probe-transported and electrostatic-force-attached CdSe (red), CdS (green) and ZnO (ultraviolet) nanowire arrays. ${ }^{[134]}$ Single-color, dual-color and three-color lasing are realized by adjusting the excitation power to this hybrid system. Gardiner, Damian, et al. directly painted the red, green and blue thin layers of liquid crystals on the substrate and after the excitation of a $430 \mathrm{~nm}$ pulsed laser, white light emission was observed as the emission of the three layers are in different wavelengths. ${ }^{[135]}$ In general, the proposed approaches of multicolor emission are sophisticated, not flexible and thus need to be further developed.

In this work, the inkjet printing technique is utilized for the fabrication of hemispheres by employing the microplotter. Precise control of both the size and position of the hemispherical microlasers are realized and high-quality WGM lasing is observed. In addition, multicolor laser emission is obtained in a single hemisphere by simply adjusting the donor to acceptor ratio within the cavity. Meanwhile, the underlying nonradiative energy transfer process, or FRET, is explored and discussed. This work not only increases the flexibility and broadens the applications of the hemispherical microlasers, but also provides a new insight into a facile and flexible approach to achieve multicolor lasing for illumination and display.

\subsection{Inkjet printing of the dye-doped hemispherical microlasers}

The gain media for the hemispherical microlasers are chosen to be Rhodamine B (RhB, $\mathrm{C}_{28} \mathrm{H}_{31} \mathrm{ClN}_{2} \mathrm{O}_{3}$, red emission, from Sigma-Aldrich) and Coumarin 540A (C540A, $\mathrm{C}_{16} \mathrm{H}_{14} \mathrm{~F}_{3} \mathrm{NO}_{2}$, green emission, from Exciton). The molecular structures of 
the two types of dyes, the absorption and emission spectrum of RhB molecules, and the emission position of the C540A are shown in Fig. 3.1. ${ }^{[136,137]}$ It can be clearly seen from Fig. 3.1 (b) and (d) that the emission spectrum of C540A overlaps with the absorption spectrum of RhB, which makes them appropriate donors and acceptors for FRET.<smiles>CCN(CC)c1ccc2c(-c3ccccc3C(=O)O)c3ccc(=[N+](CC)CC)cc-3oc2c1</smiles>
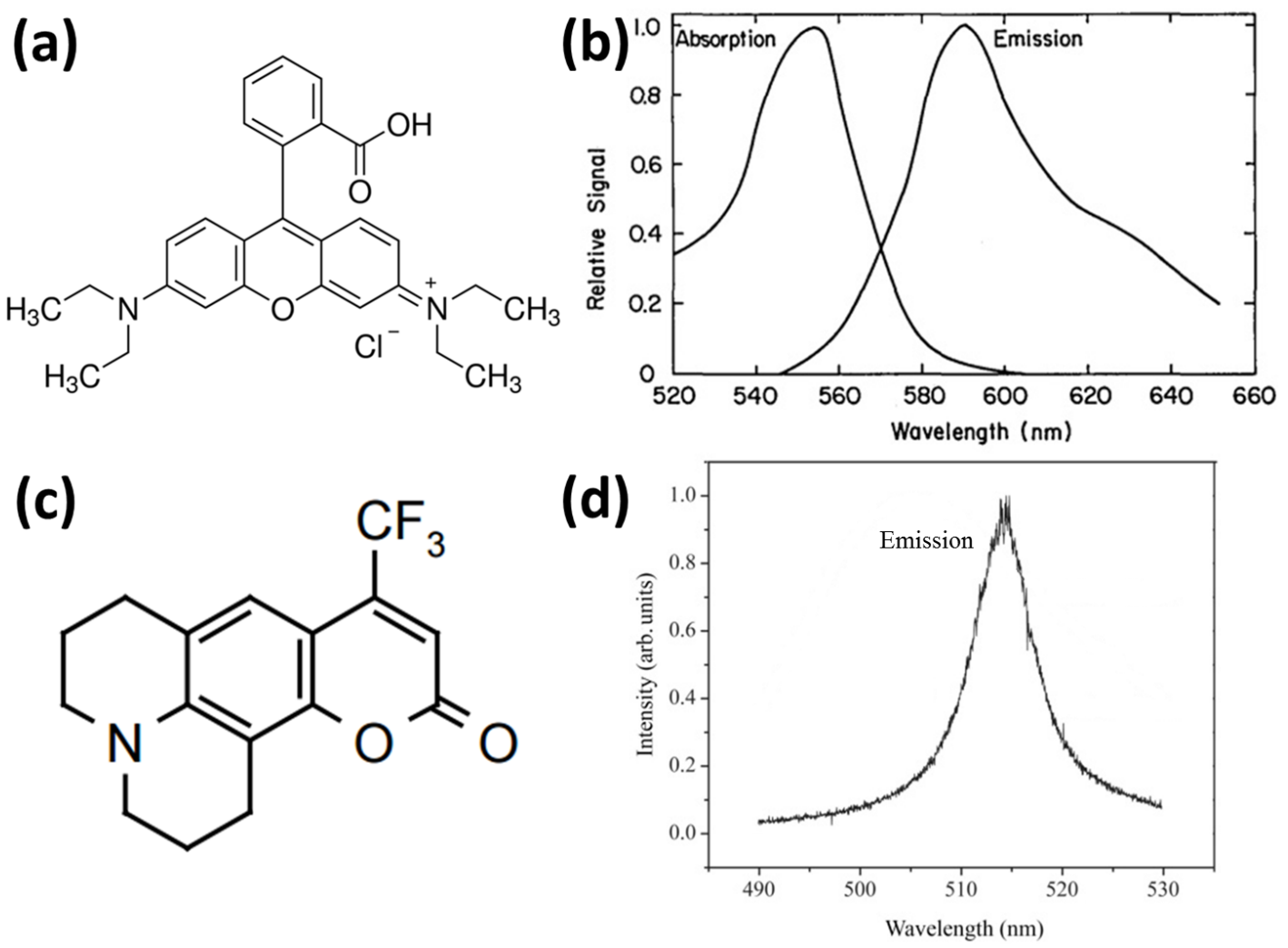

Figure 3.1. Properties of the Rhodamine B and Coumarin 540A dye molecules. (a) Molecular structure of the Rhodamine B. (b) Absorption and emission spectra of the Rhodamine B. ${ }^{[136]}$ (c) Molecular structure of the Coumarin 540A. (d) Emission spectrum of the Coumarin 540A. ${ }^{\text {[137] }}$

Initially, the $\mathrm{RhB}$ and $\mathrm{C} 540 \mathrm{~A}$ were dissolved in dichloromethane $\left(\mathrm{CH}_{2} \mathrm{Cl}_{2}\right.$, $0.15 \mathrm{~mL}$, purity $99.76 \%)$ separately with the same number of molecules $(0.05 \mathrm{mmol}$, $15.5 \mathrm{mg}$ for $\mathrm{C} 540 \mathrm{~A}$ and $24 \mathrm{mg}$ for $\mathrm{RhB}$ ). Then the C540A solution was reproduced for seven times with the same amount of dyes and $\mathrm{CH}_{2} \mathrm{Cl}_{2}$. After adding 0.8, 1, 1.2, 
1.6, 2.4, 4.9, and $24 \mathrm{mg} \mathrm{RhB}$ separately to the seven reproduced C540A solutions, the donor $(\mathrm{C} 540 \mathrm{~A})$ and acceptor $(\mathrm{RhB})$ were mixed together with the molar ratios $\left(\mathrm{n}_{\text {donor }}\right.$ : $\mathrm{n}_{\text {acceptor }}$ ) of 1:0.03, 1:0.04, 1:0.05, 1:0.07, 1:0.1, 1:0.2, and 1:1 in order to study the energy transfer processes and the multicolor lasing performances of the dye molecules. Finally, epoxy resin (Araldite 506 from Sigma-Aldrich), which was chosen to be the host material of the hemispherical microlasers due to the high viscosity, optically transparency and inert thermal properties, was added to the nine samples (including the pure donor and the pure acceptor solutions), with a mass of $600 \mathrm{mg}$. After oscillation in the ultrasonic vibrator for $30 \mathrm{~min}$, the sample solutions were ready for fabricating hemispherical microlasers.

The distributed Bragg reflectors (DBR) were chosen to be the substrates for the self-assembled hemispheres. As illustrated in Fig. 3.2 (a), the commercial DBR substrates are made by alternating $\mathrm{SiO}_{2}$ and $\mathrm{TiO}_{2}$ quarter-wave layers ${ }^{[28]}$ and have ultra-high reflectivity (larger than 99.5\%) from 520 to $680 \mathrm{~nm}$, which can help the hemispherical microlasers to confine the light in the vertical direction and obtain high $Q$ factors. ${ }^{[10]}$ Then a thin layer of transparent hydrophobic film $(1 \mathrm{H}, 1 \mathrm{H}, 2 \mathrm{H}$, 2H-perfluoroo-ctyltriethoxysilane) was spin-coated on a selected DBR substrate in order to get circular hemispheres during the self-assembling processes (Fig. 3.2 (b)). Finally, the hemispherical microlasers were inkjet-printed on the surface of the hydrophobic layer via the pecking technique of the microplotter (Fig. 3.2 (c)). 

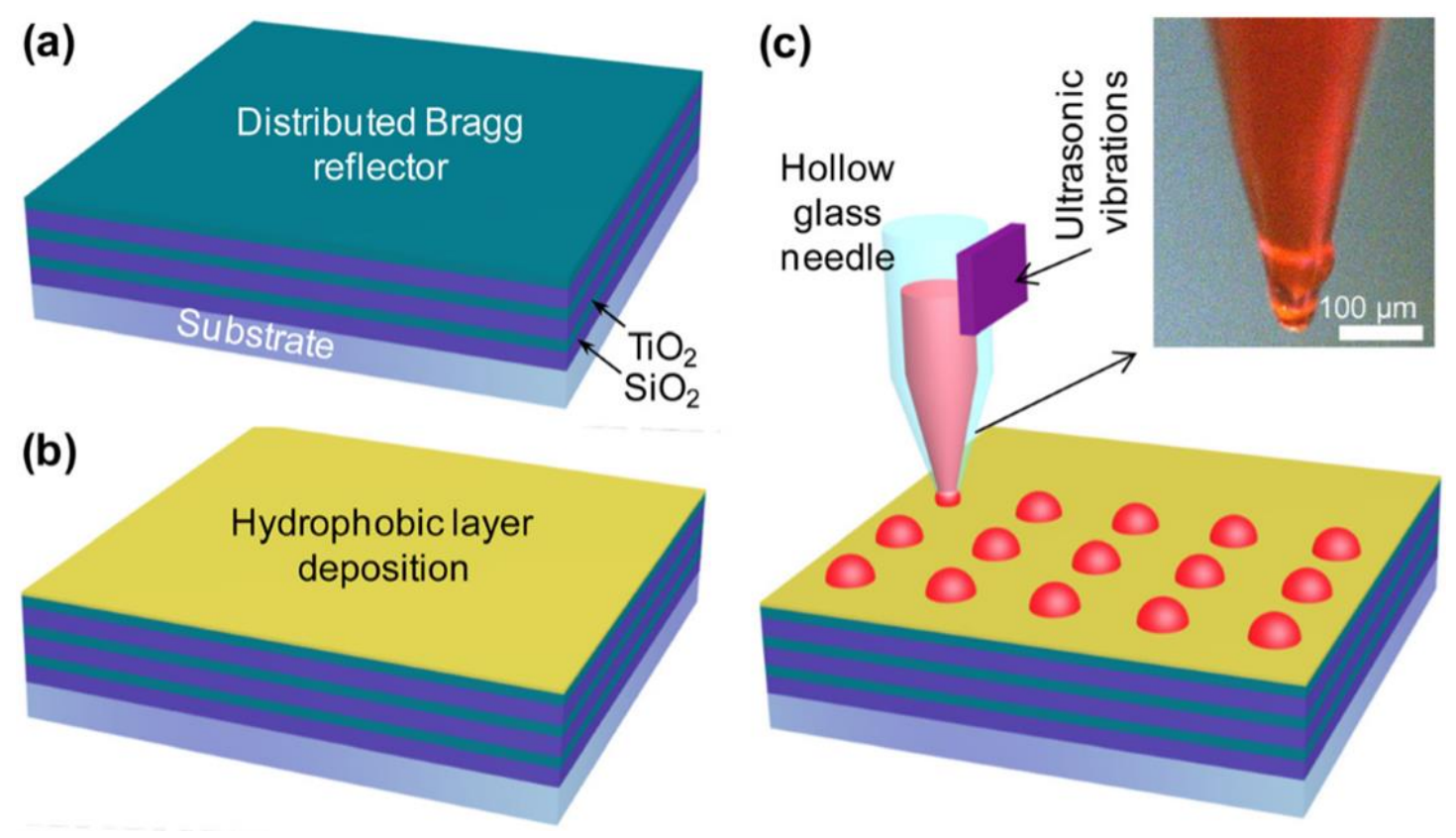

Figure 3.2. The precise fabrication of the hemispherical microlasers.

(a) Schematic of the DBR substrate. (b) The deposition of the hydrophobic layer. (c) Fabrication of the hemispheres via the dipping technique of the microplotter. ${ }^{[119]}$

The optical images of the fabricated hemisphere arrays are shown in Fig. 3.3 (a) and (b). It can be seen clearly that dipping technique dramatically improves the control of both the position and the size of the hemispheres during fabrication. The high stepping resolution of the microplotter enables the resonators to be positioned with precision of $1 \mu \mathrm{m}$ and the diameters of the hemispheres can be controlled via dispensers with different dimensions (30 and $80 \mu \mathrm{m}$ head-diameters for the dispensers used in Fig 3.3(a) and (b), respectively, and resulted in hemisphere-diameters of 35 and $85 \mu \mathrm{m})$. In order to verify the reproducibility and homogeneity of the dipping technique, diameters were measured for the 173 hemispherical microlasers fabricated by the dispenser with the head-diameter of $30 \mu \mathrm{m}$. The statistical distribution is reported in Fig. 3.3 (c) and shows that the majority of the samples (about 97\%) exhibit diameters of $35 \pm 3 \mu \mathrm{m}$ and all the samples are in the range of $35 \pm 4 \mu \mathrm{m}$ 
(diameter). Therefore, the precise fabrication of the hemispherical microlasers is achieved by employing the microplotter.
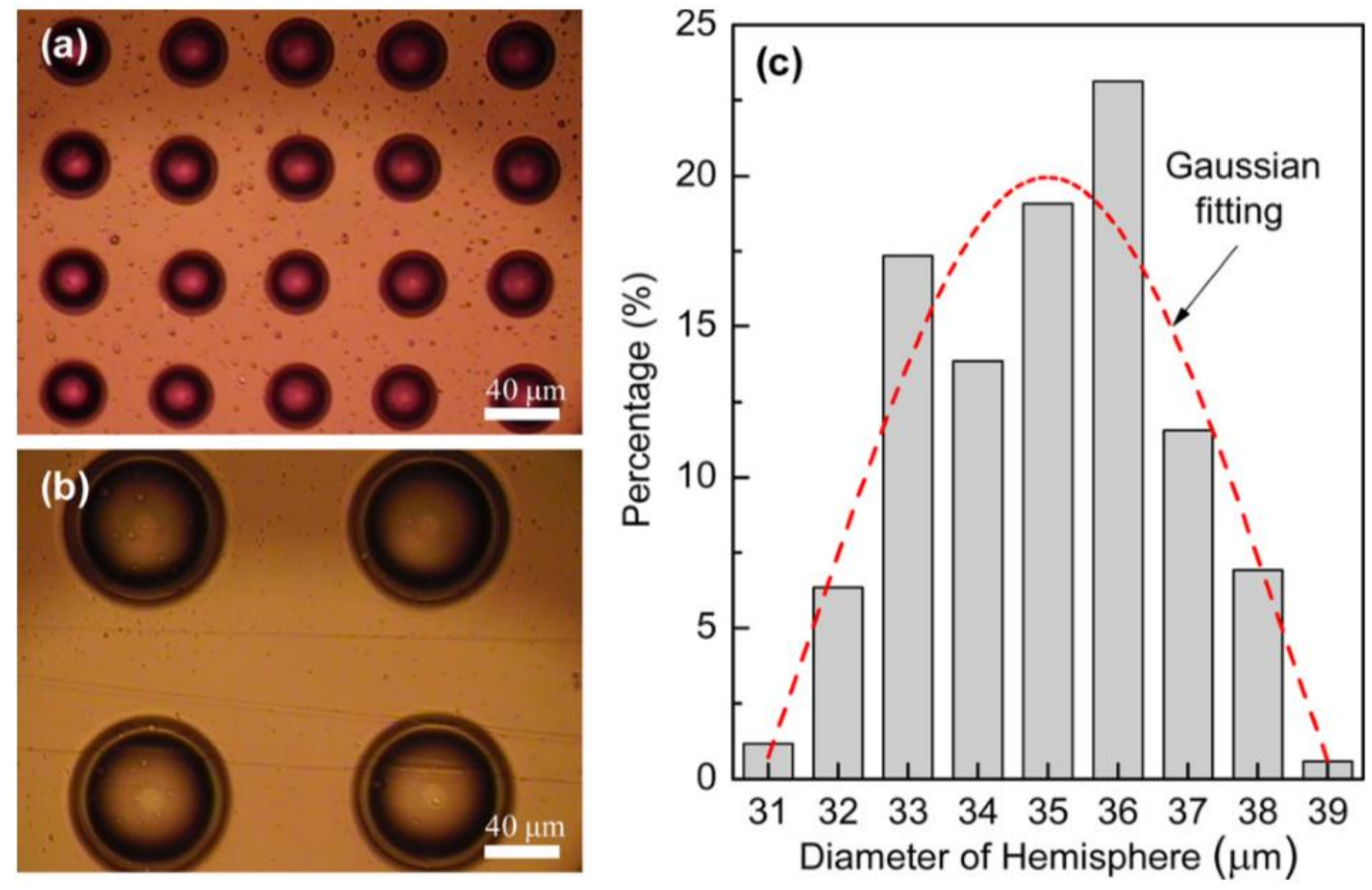

Figure 3.3. Hemispherical microlaser arrays and the statistical distribution of the diameters.

(a) Hemispheres fabricated by a dispenser with the head-diameter of $30 \mu \mathrm{m}$. (b) Hemispheres fabricated by a dispenser with the head-diameter of $80 \mu \mathrm{m} .(\mathrm{c})$

Diameter-distribution of the 173 hemispheres fabricated by the $30 \mu m$-diameter dispenser. ${ }^{[119]}$

\subsection{Multicolor lasing from the dual-dye-doped hemispheres}

The optical properties of the hemispherical microlasers were investigated by the $\mu$-PL system introduced in section 2.5. It is worth noting that the excitation source in this work was chosen to be a pulsed Nd : YAG laser (pulse width: 5-6 ns, repetition rate: $20 \mathrm{~Hz}$ ) with the wavelength of $420 \mathrm{~nm}$. Although the absorption of the $\mathrm{RhB}$ molecules is relatively low at $420 \mathrm{~nm}$, it is important to keep the pumping wavelength consistent for the study of the energy transfer processes between the donors and 
acceptors. Fig. 3.4 (a) and (b) exhibit the emission spectra from single-dye-doped hemispheres with diameters of $85 \mu \mathrm{m}$ and $57 \mu \mathrm{m}$, respectively. It can be seen that under low pumping intensities, only spontaneous emission is observed for both the C540A-doped (green emission, Fig. 3.4 (a)) and the RhB-doped (red emission, Fig. 3.4(b)) hemispheres. With the increase of the pumping intensity, lasing dominates the emission as proved by the sharp peaks in the spectra and the bright rings near the circular boundaries of the hemispheres in the optical images (insets of Fig. 3.4).
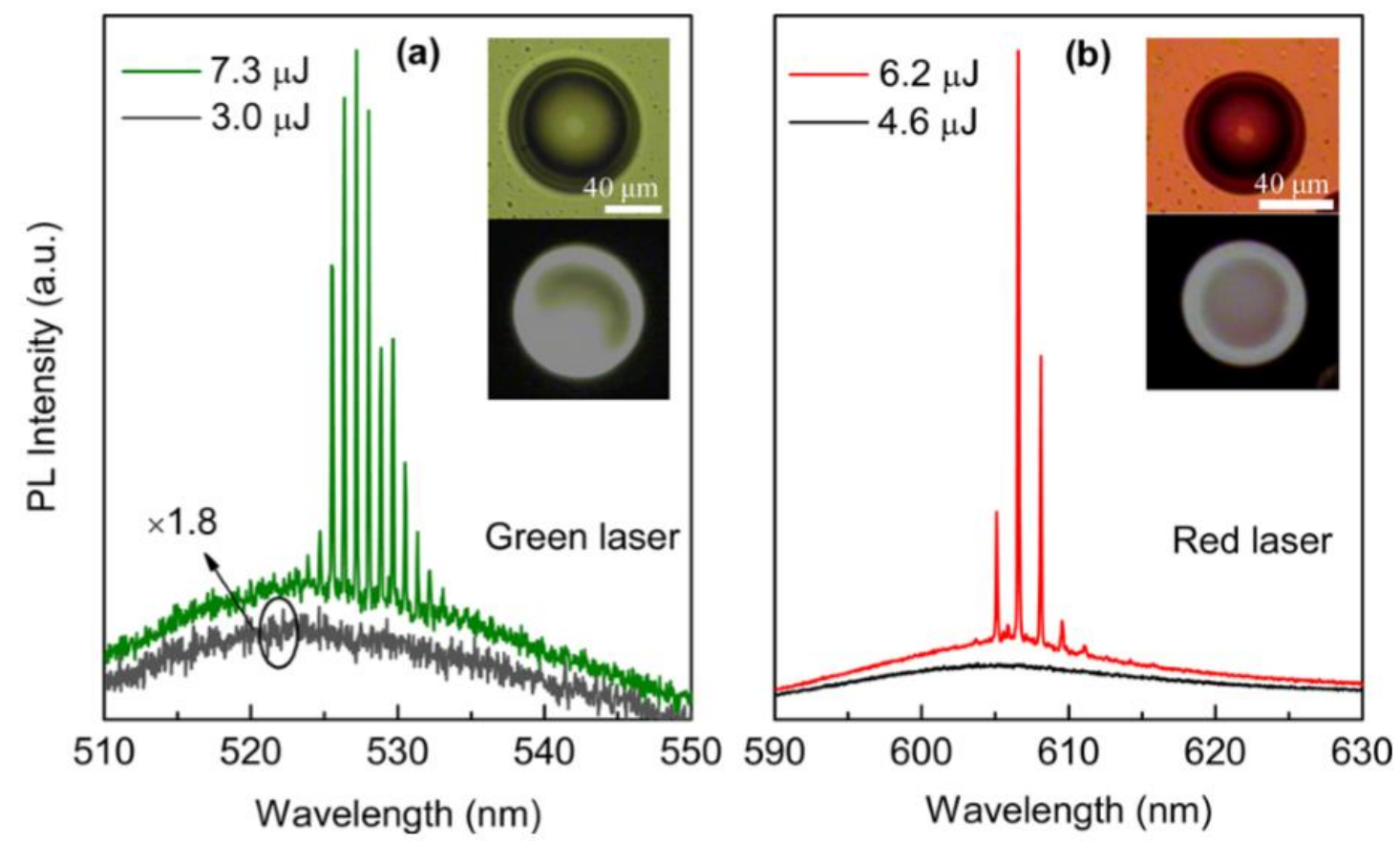

Figure 3.4. Laser emission from the single-dye-doped hemispheres.

(a) The spontaneous emission and green lasing from the C540A-doped hemisphere with a diameter of $85 \mu \mathrm{m}$. (b) The spontaneous emission and red lasing from the $R h B$-doped hemisphere with a diameter of $57 \mu \mathrm{m}$. The insets show the optical images of the hemispheres under normal illumination (upper ones) and excitation with high pumping intensities (lower ones). ${ }^{[119]}$

As reported in the previous works, the hemispherical microlasers can sustain high-quality WGM lasing. ${ }^{[10,28,47]}$ Besides, the clear mode spacing of the peaks indicates the existence of only one set of modes, which are ascribed as fundamental 
modes $(r=1, l=m)$ due to their relatively high $Q$ factors comparing with the higher order modes. Therefore, the lasing spectra are analyzed by the basic WGM theory (Eq. (1.6)). After substituting $\mathrm{n}_{\text {eff }}=1.41$ and $\mathrm{D}=59.69 \mu \mathrm{m}$ into Eq. (1.6), the longitudinal mode numbers $m$ fit well with the integers range from 412 to 415 for the lasing peaks in Fig. 3.4 (b). In addition, the calculated FSRs (based on WGM theory, Eq. (1.36)) for the green and red lasing are 0.75 and $1.48 \mathrm{~nm}$, respectively, which are close to the measurements from the spectra $(0.8 \mathrm{~nm}$ for green and $1.5 \mathrm{~nm}$ for red). Meanwhile, the FWHM (full width at half maximum) of the lasing peaks is around $0.11 \mathrm{~nm}$, which is better than the previously reported microresonators produced by the inkjet printing methods. ${ }^{[138,139]}$

As for a dual-dye-doped hemisphere, various types of lasing including pure donor lasing (green), pure acceptor lasing (red), as well as dual color lasing can be achieved by adjusting the molar ratios of donor (C540A) to acceptor (RhB). As shown in Fig. 3.5 (a), when donor : acceptor $>1: 0.05$, which indicates the low concentration of the acceptor molecules, green lasing is observed, together with the spontaneous emission of the acceptor molecules in the orange-red region. In this case, the gain that the acceptors obtain from the excitation and the energy transfer processes is not high enough for lasing. In contrast, when donor : acceptor $<1: 0.1$, that is, at relatively low concentration of the donor molecules, only red lasing occurs and the emission of the donors is inhibited due to the energy transfer processes from the donors to acceptors (Fig. 3.5 (b)). It is important to note that in comparison with the single-dye-doped red lasing, the lasing threshold of the dual-dye-doped (donor : 
acceptor $=1: 1)$ hemispheres with the same acceptor concentration and similar sizes can be decreased by 2.5 times due to the FRET, which increases the overall absorption efficiency. When $1: 0.1<$ donor : acceptor < $1: 0.05$, dual color lasing (green: 515-535 nm, orange-red: 585-605 nm) can be achieved (Fig. 3.5 (c)). In this case, the gain of the donors is sufficient to support lasing although a portion of the energy is transferred to the acceptors. The reflectivity of the DBR substrate, which covers all the lasing region of the microlaser, is also illustrated in Fig. 3.5 (c), indicating the strong optical confinement in the vertical direction.
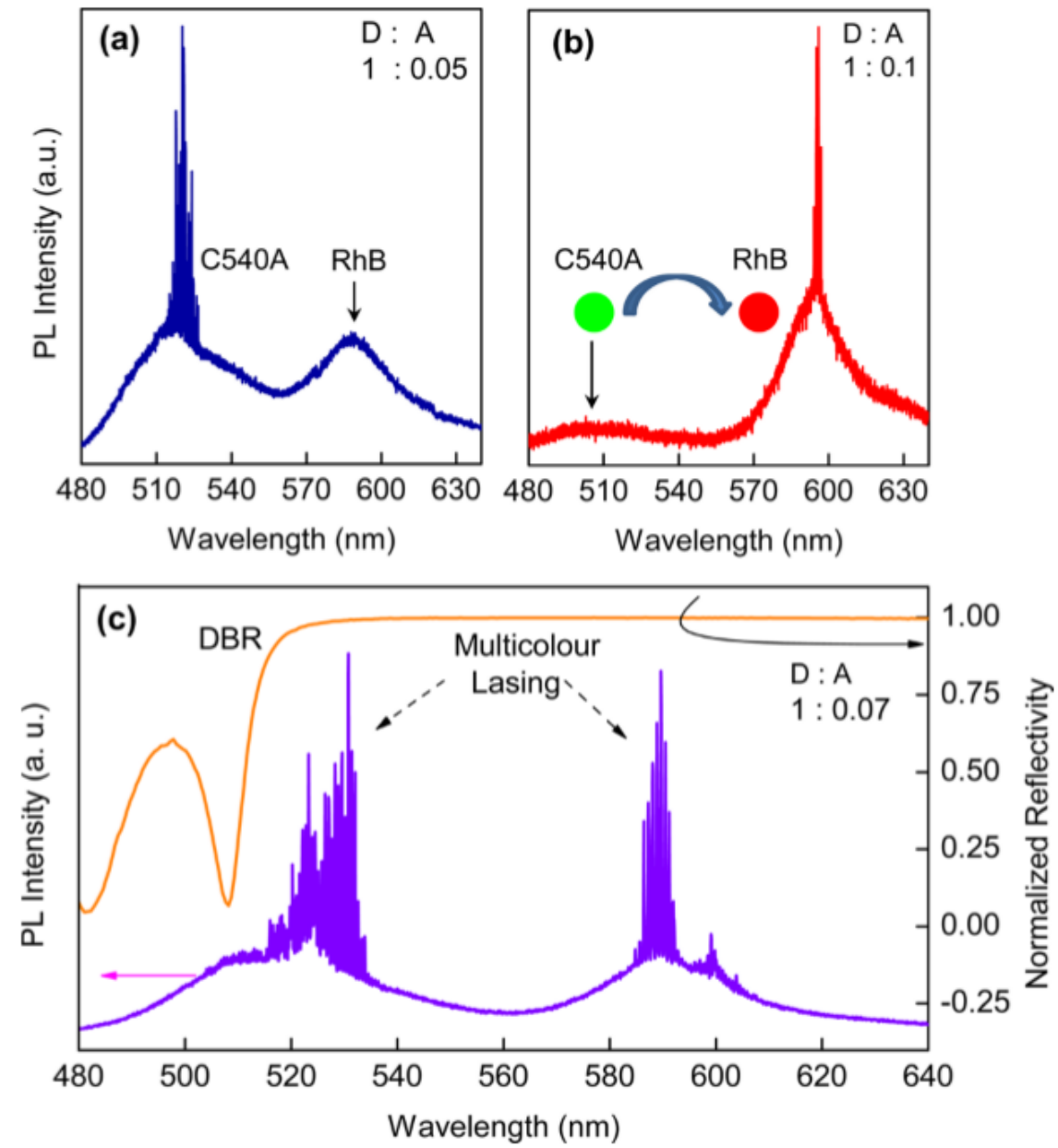

Figure 3.5. Multicolor lasing from a dual-dye-doped hemispherical microlaser. (a) Donor lasing with the low concentration of acceptors. (b) Acceptor lasing induced by energy transfer processes. (c) Multicolor lasing with an appropriate mole ratio of donor to acceptor. ${ }^{[119]}$ 


\subsection{Investigation of the energy transfer processes}

In order to explore the energy transfer processes within the dual-dye-doped hemispheres, steady-state photoluminescence (PL) measurements (based on solutions, without microcavity) were carried out by utilizing a Xeon lamp at the wavelength of $420 \mathrm{~nm}$. The normalized PL intensities are shown in Fig. 3.6. In order to get an intuitive comparison of the PL intensities, the spectrum of the pure C540A solution is normalized according to the donor emission, while other spectra of the dual-dye-doped solutions are normalized with regard to the acceptor emission. The gradual dropping of the donor PL intensity $(\sim 80 \%$ at donor $:$ acceptor $=1: 0.03$, and $\sim 7 \%$ at donor : acceptor $=1: 1)$ can be observed clearly with the increase of the acceptor concentration, which indicates the energy transfer processes between the two types of dye molecules.

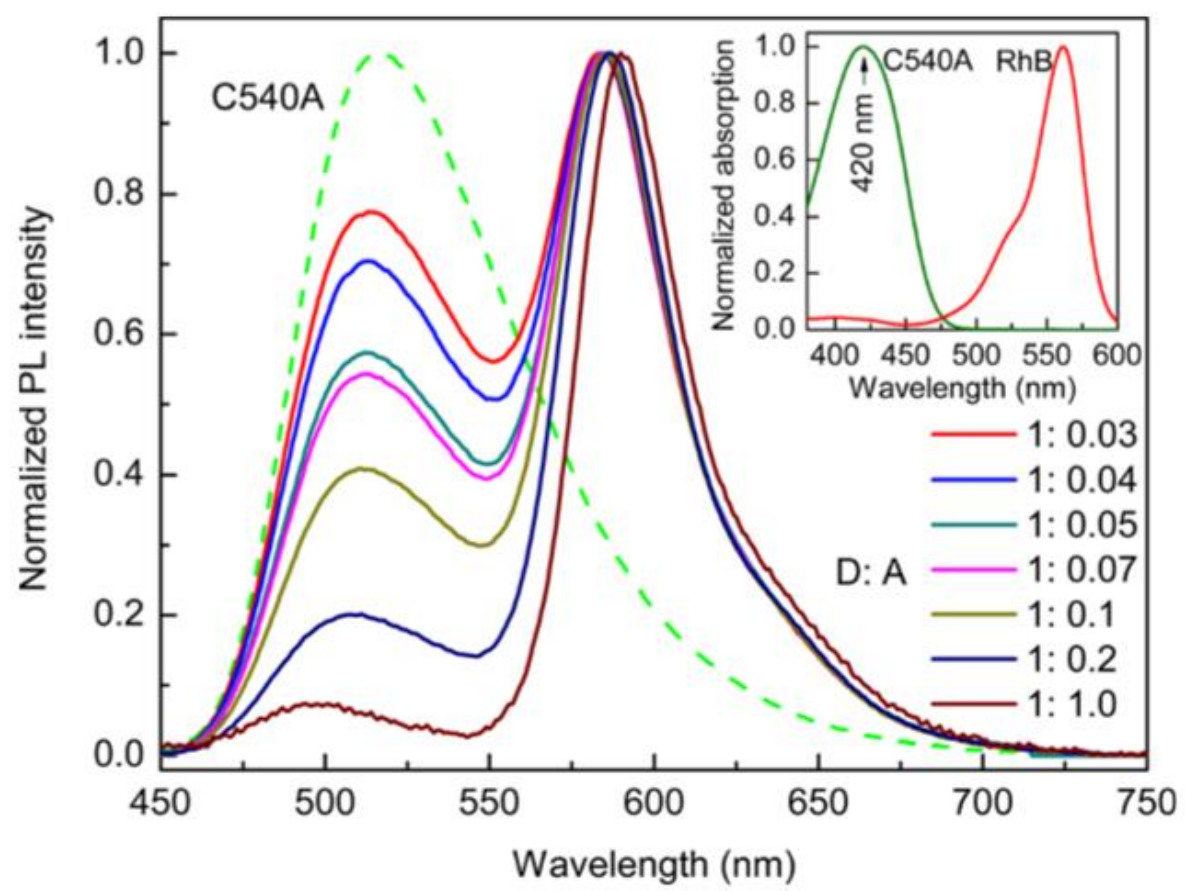

Figure 3.6. Normalized PL intensities of the dual-dye-doped solutions.

The dashed green line depicts the PL emission of the pure donor solution and the inset shows the absorption spectra of the two types of dye molecules. ${ }^{[119]}$ 
Generally, the energy transfer between the donors and acceptors can be realized by the nonradiative process (FRET) or the radiative process. ${ }^{[131]}$ In FRET, the excited donor molecules emit virtual photons that are immediately absorbed by the acceptor molecules, which are within the Förster distance with the donor molecules. ${ }^{[140]}$ As the virtual photons are undetectable, the FRET is nonradiative and the energy transfer is considered to be accomplished by the dipole-dipole coupling of the chromophores. In contrast, in the radiative energy transfer process, the excited donors emit real photons via radiative recombination and then the photons are reabsorbed by the acceptors. This process is radiative because the emitted photons are detectable. ${ }^{[131]}$ In order to distinguish the radiative and nonradiative energy transfer processes, the TRPL measurements (section 2.6) are normally performed based on the donor lifetimes: ${ }^{[141]}$

$$
\begin{gathered}
\frac{1}{\tau_{\text {donor }}}=\frac{1}{\tau_{\text {radiative }}}+\frac{1}{\tau_{\text {nonradiative }}}, \quad \text { without acceptors } \\
\frac{1}{\tau_{\text {donor }}^{\prime}}=\frac{1}{\tau_{\text {radiative }}^{\prime}}+\frac{1}{\tau_{\text {nonradiative }}^{\prime}}+\frac{1}{\tau_{\text {transfer }}}, \quad \text { with acceptors }
\end{gathered}
$$

where $\tau_{\text {donor }}^{\prime}$ and $\tau_{\text {donor }}$ are the donor lifetimes with and without the presence of acceptors, respectively. $\tau_{\text {radiative }}^{\prime}\left(\tau_{\text {radiative }}\right)$ and $\tau_{\text {nonradiative }}^{\prime}\left(\tau_{\text {nonradiative }}\right)$ are the decay times of radiative recombination and nonradiative recombination in the two cases and $\tau_{\text {transfer }}$ stands for the nonradiative energy transfer rate from the donors to the acceptors. The radiative and nonradiative recombination decay times are expected to be similar in the two cases $\left(\tau_{\text {radiative }}^{\prime} \approx \tau_{\text {radiative }}, \tau_{\text {nonradiative }}^{\prime} \approx\right.$ $\tau_{\text {nonradiative }}$ ), and therefore, the donor lifetime in Eq. (3.2) is shorter than the one in Eq. (3.1) $\left(\tau_{\text {donor }}^{\prime}<\tau_{\text {donor }}\right)$ because of the nonradiative energy transfer process $\left(\tau_{\text {transfer }}\right)$, which provides an additional relaxation pathway for the carriers. ${ }^{[141]}$ 
In the TRPL measurement of this work, the pure donor solution and several dual-dye-doped solutions with different donor to acceptor ratios were pumped by a tunable femtosecond laser (pulse duration: $100 \mathrm{fs}$, repetition rate: $1 \mathrm{kHz}$ ) at the wavelength of $420 \mathrm{~nm}$. Then the emission around $520 \mathrm{~nm}$ was collected via a streak camera (temporal resolution: $50 \mathrm{ps}$ ) and the results are shown in Fig. 3.7. The lifetime curves can be described by the bi-exponential decay function with reconvolution: ${ }^{[131]}$

$$
I(t)=\int_{-\infty}^{t} \operatorname{IRF}\left(t^{\prime}\right) A e^{\left(t^{\prime}-t\right) / \tau} d t^{\prime},
$$

where $I(t)$ is the intensity of the component at time $t, I R F$ is the response function of the equipment, $A$ is the initial intensity of the component $(t=0)$, and $\tau$ is the lifetime. It can be concluded from the extracted donor lifetimes (shown in Fig. 3.7) that dramatic lifetime-drop occurs with the increasing of the acceptor concentration (2.4 ns for the pure donor solution and $0.4 \mathrm{~ns}$ for the solution with donor : acceptor $=$ $1: 1)$, which proves the existence of FRET. 


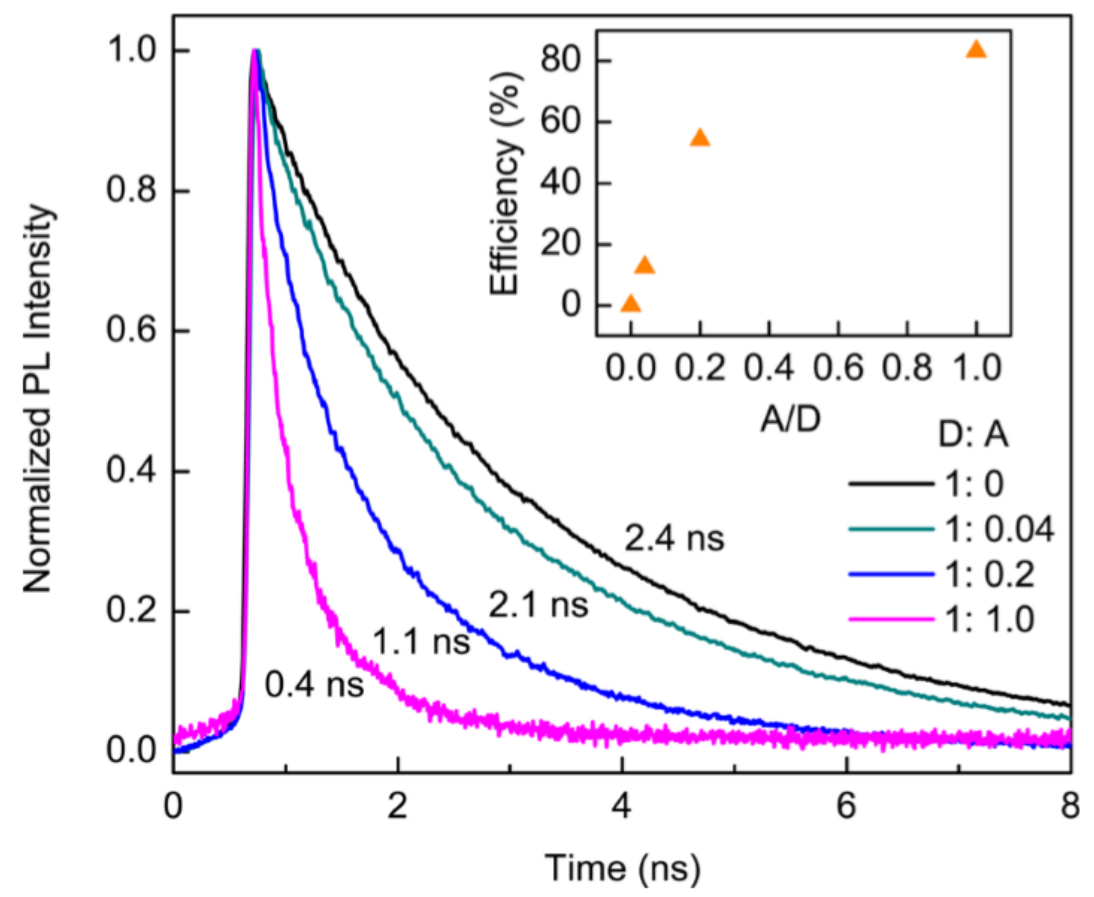

Figure 3.7. TRPL of the donors (C540A) with and without the acceptors (RhB).

The lifetimes are shown beside each curve and the inset shows the efficiency of FRET with respect to the mole ratios of acceptor to donor. ${ }^{[119]}$

The efficiency of FRET, or $\eta_{F R E T}$, can be obtained by: ${ }^{[142,143]}$

$$
\eta_{F R E T}=1-\frac{\tau}{\tau_{0}}
$$

where $\tau$ and $\tau_{0}$ are the lifetimes of the donors with and without the doping of the acceptors, respectively. With the acceptor to donor ratio varying from 0.04 to 1 , which causes a decrease in molecular distance between the donors and acceptors, the efficiency of FRET increases from $12.5 \%$ to $83.3 \%$ (depicted in the inset of Fig. 3.7). Therefore, the decreased lasing threshold of the dual-dye-doped hemispheres with the same acceptor concentration and size as the single-dye-doped ones is attributed to the high-efficient FRET. As a result, the wavelength-controllable, energy-efficient, printable and flexible multicolor hemispherical microlasers are theoretically promising candidates for the applications in illumination and display. 


\subsection{Summary and outlook}

In summary, the hemispherical microlasers are inkjet-printed on the substrate with precise control of both the position and the size by employing the advanced pecking technique of the microplotter. High-quality single-color WGM lasing is obtained in both the single-dye-doped and the dual-dye-doped microlasers with the $Q$ factors in the order of $10^{3}$. Meanwhile, by adjusting the molar ratios of the embedded donors and acceptors, multicolor lasing at 515 to $535 \mathrm{~nm}$ (green) and 585 to $605 \mathrm{~nm}$ (orange-red) is achieved. Detailed studies reveal that the nonradiative FRET is the dominant energy transfer process between the donors and acceptors and can reach efficiency up to $83.3 \%\left(\mathrm{n}_{\text {donor }}: \mathrm{n}_{\text {acceptor }}=1: 1\right)$. In addition, the high-efficient FRET enables the dual-dye-doped hemispheres to exhibit a 2.5 times lower threshold than the single-dye-doped ones for the pure acceptor lasing.

This work not only introduces the advanced fabrication technique that can be widely applied in controllable, large-scale manufacturing and integration of hemispherical microlasers, but also demonstrates the possibility of FRET-induced multicolor lasing, or wavelength tuning by doping two types of organic dyes into a single microcavity, which can be further explored by embedding more kinds of dye molecules and studying the more complex energy transfer processes for specific applications. Even white color lasing, which is significant in illumination and display, can be achieved by mixing the red, green and blue dyes in the proposed simple-fabricated, precise-controlled, energy-efficient and flexible-doped hemispherical microlasers. 


\section{Spirally Drawn Whispering Gallery Mode Microfiber Arrays and the Sensing Applications}

\subsection{Introduction and motivation}

Conventionally, microfibers are widely applied as waveguides to guide the signals in optoelectronics and are rarely studied for their lasing performances. ${ }^{[102]}$ The few reported lasing works using active microfibers are mainly based on the Fabry-Perot mechanism by utilizing the two ends of the fibers as high-reflective mirrors, which induces large optical loss and results in low $Q$ factors. ${ }^{[103-105]}$ However, as the circular cross-sections of the microfibers make them ideal microcavities to sustain WGMs, after WGM lasing from dye-doped polymer microfibers was reported by Prof. Sun's group, ${ }^{[48]}$ they quickly developed applications like gas sensing, refractive index sensing, and Vernier effect study. ${ }^{[2,45,48]}$ It is known that due to the low efficiency of free-space coupling, the traditional WGM microcavities and microlasers need waveguides, or tapered fibers to couple the light in and out of the cavities, which is critical to the coupling distance and requires precise control with sophisticated equipment. ${ }^{[144]}$ In contrast, the cylindrical geometry along the longitudinal direction allows the active microfibers to act as waveguides simultaneously, which is much more convenient and flexible than other WGM configurations. ${ }^{[48]}$ In addition, the soft-approached microfibers are promising in sensing applications because of their superior optical performances, simple fabrication, low cost, mechanical flexibility, straightforward sample-excitation and 
signal-collection routes. ${ }^{[6,32,45,48]}$ Nevertheless, the reported fabrication methods for polymer microfiber lasers are mainly based on manual processes with coarse resolutions, which are low in efficiency and lack control of the sizes and positions of the microfibers. This has hindered applications of the microfiber lasers, especially in large-scale integration and on-chip cascade.

On the other hand, the strain sensors, which detect small variations in strain and provide apparent changes in the feedback, are building blocks in abundant applications such as pressure sensing in fluid mechanics, wall shear stress sensing, human-motion and structural deformation detection, etc. ${ }^{[145-148]}$ Typically, the feedback provided by strain sensors relies on optical or electrical property variations caused by the external forces or structural perturbations, as exemplified by the semiconductor-based piezoresistive strain sensor, whose electrical resistance varies with different forces applied, and the fiber Bragg grating (FBG) sensor, whose spectrum shifts with the force-induced period change of the Bragg gratings. ${ }^{[149-152]}$ However, although the WGM microcavities and microlasers hold the advantages of high $Q$ factors, small mode volumes, and large optical densities which enable outstanding optical performances for sensing, ${ }^{[6]}$ their applications as strain sensors are not well-developed, probably due to the sensitivity limitation brought by the solid nature of the conventional cavity materials. However, the emergence of the soft-approached WGM microcavities and microlasers breaks this bottleneck and increases the sensitivity because of the intrinsic elastic nature of the polymer materials. ${ }^{[45,118]}$ Furthermore, comparing with the FBG strain sensors which only 
sense the perturbations along the fiber axis, the WGM configurations are much more flexible due to the multi-dimensional sensing induced by the symmetry of the circular WGM cross-sections. ${ }^{[53,153]}$ Thus, the soft-approached WGM candidates are promising in the field of strain sensors.

In this work, polymer microfiber laser arrays are fabricated in the elastic polydimethylsiloxane (PDMS) matrix by the spiral drawing technique. With the help of the microplotter, the sizes and the positions of the microfibers can be well-controlled. Besides high-quality WGM lasing, the redshift of the lasing peaks is observed under external forces applied on the PDMS matrix. Detailed studies illustrate that the whole systems (microfiber arrays buried in PDMS matrix) can be utilized as reliable strain and force sensors with sensitivity of $0.45 \mathrm{pm} / \mu \varepsilon$ (strain), $5.66 \mathrm{~nm} \cdot \mathrm{N}^{-1}$ (force) and the limit of detection down to $1.76 \times 10^{-4} \varepsilon$ (strain), and $0.014 \mathrm{~N}$ (force). This work not only proposes a controllable and efficient fabrication technique for the large-scale microfiber lasers, but also broadens the applications of the soft-approached WGM microcavities in the field of strain sensing, which could be further exploited for structural health monitoring or for the detection of multi-point perturbations.

\subsection{Spiral drawing of the microfiber lasers}

The host materials for the microfibers were mainly epoxy resin (Araldite 506), which is transparent, doping-flexible, viscous, optically and thermally inert, ${ }^{[28]}$ and polymethylmethacrylate (PMMA, from Sigma-Aldrich), which is used to modulate 
the viscosity of the mixture. ${ }^{[4]}$ The $300 \mathrm{mg}$ PMMA powder, together with the $20 \mathrm{mg}$ gain medium (Rhodamine B, or RhB, from Sigma-Aldrich), were firstly dissolved in $5 \mathrm{~mL}$ of dichloromethane $\left(\mathrm{CH}_{2} \mathrm{Cl}_{2}\right.$, purity $\left.99.76 \%\right)$. After 20 minutes of ultrasonic vibration, $750 \mathrm{mg}$ liquid epoxy resin was mixed with the solution to reach the mass-ratio of $m_{P M M A}: m_{\text {epoxy }}=0.4$. Finally, another round of ultrasonic vibration was applied to the mixture for 30 minutes and then, the homogeneous host solution for the microfiber lasers was ready for spiral drawing.

The microplotter (section 2.1) was used for spiral drawing because of its high stepping-resolution. As illustrated in Fig. 4.1 (a), the tip of the microdispenser was firstly immersed in the solution droplet to absorb enough host materials. Due to the enhanced viscosity induced by the PMMA, a cylindrical precursor would be drawn out of the droplet when the microdispenser was pulled up quickly with a height of $h$ (Fig. 4.1 (b)). Then a rotational motor with the uncured PDMS (Sylgard 184 from Dow Corning) matrix fixed at the center was approached to the precursor by an X-Y-Z stage (Fig. 4.1 (c)). The PDMS matrix, which is elastic, transparent, stable, and can serve as the protection layer of optical and electrical devices, ${ }^{[45]}$ was initially coated on a glass substrate $(30 \mathrm{~mm} \times 30 \mathrm{~mm} \times 1 \mathrm{~mm})$ with the base to curing ratio of $10: 1$ (volume ratio). After spiral rotation of the motor in a short time, microfiber arrays were buried in the PDMS matrix and stuck on the back of the substrate simultaneously (Fig. 4.1 (d)). The next step was to bake the sample substrate at $50{ }^{\circ} \mathrm{C}$ for three hours to fully solidify the PDMS matrix and finally, the edges of the substrate were cut to release the microfibers stuck on the back and the cured PDMS 
matrix as well as the microfiber arrays buried inside were separated from the substrate manually.
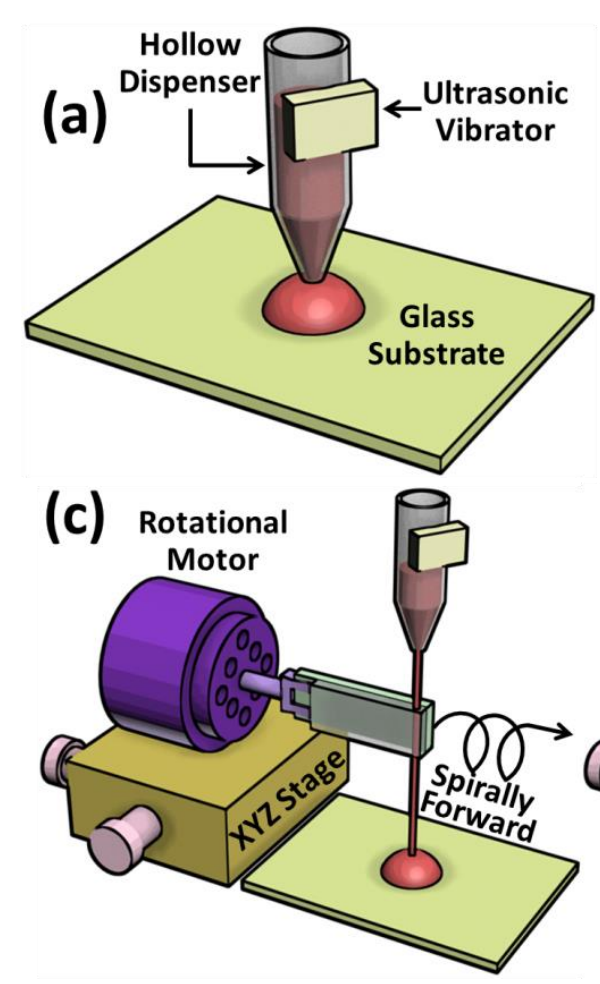

(b)

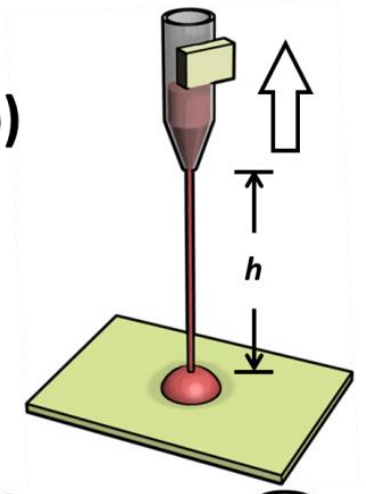

(d)

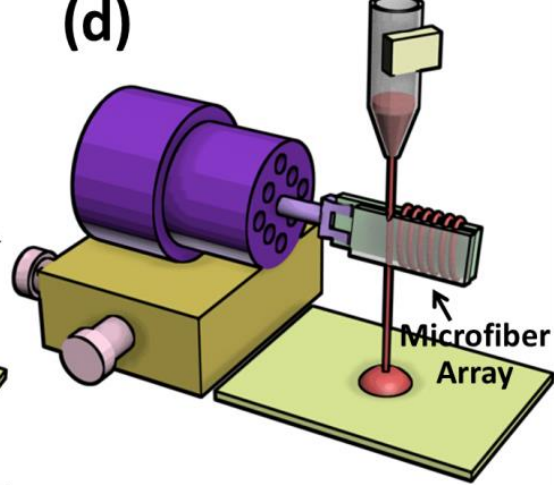

Figure 4.1. Illustration of the spiral drawing technique for the fabrication of microfiber arrays.

The optical images of the fabricated microfiber lasers are shown in Fig. 4.2 (a) and (b). It is clear to see that the periods of the microfibers are well-controlled by adjusting the stepping speed of the rotational motor. In addition, with the help of the $\mathrm{X}-\mathrm{Y}-\mathrm{Z}$ stage and the microplotter, the positions of the microfibers can be manipulated. As the solution from both the on-glass droplet and the microdispenser supplies host materials, the diameter of the cylindrical precursor remains unchanged during the spiral drawing processes, which results in uniform microfibers with the same diameter as the precursor within the PDMS matrix. Fig. 4.2 (c) reveals the relationship between the lifting height $h$ and the diameters of the fabricated microfibers. The inversely 
proportional dependence can be observed with the standard deviations around 0.24 $\mu \mathrm{m}$ for each set of samples, which indicate high homogeneity of the in-array microfibers. In addition, the high repeatability of the spiral drawing technique is examined by reproducing the microfibers for three times with the same lifting height $(10 \mathrm{~cm})$. As illustrated in Fig. 4.2 (d), the average diameters for the three sets (five microfibers in each set) are $16.07 \mu \mathrm{m}, 16.39 \mu \mathrm{m}$, and $16.40 \mu \mathrm{m}$, and the standard deviations for the samples in each set are $0.18 \mu \mathrm{m}, 0.20 \mu \mathrm{m}$, and $0.24 \mu \mathrm{m}$, respectively.
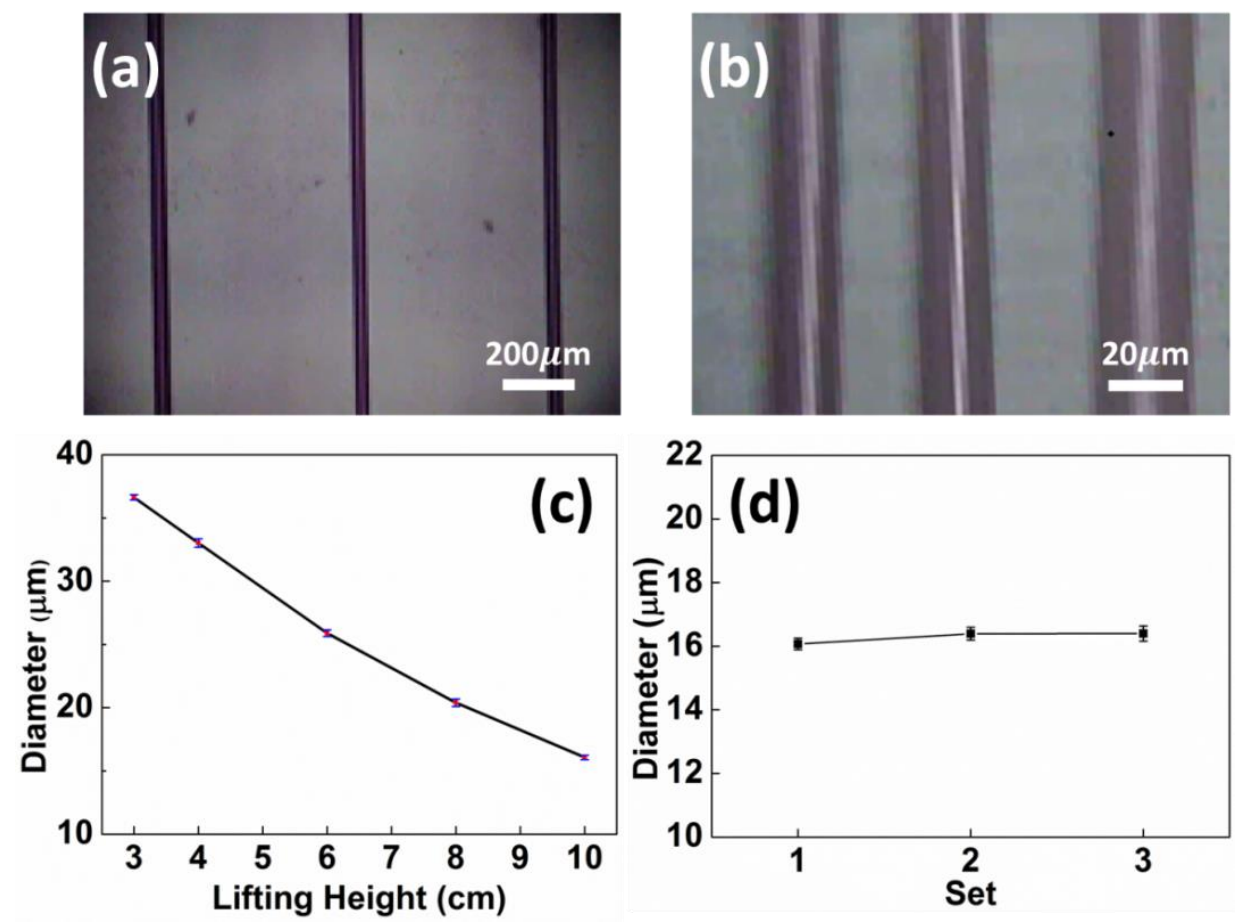

Figure 4.2. Analyses of the microfibers fabricated by the spiral drawing technique. (a)-(b) Optical images of the spirally drawn microfibers with different lifting heights of the microdispenser and advancing speeds of the rotational motor during fabrication. (c) The diameters of the microfibers as a function of the lifting heights of the microdispenser. (d) The repeatability of the spiral drawing technique examined by three independent sets with the same lifting height of $10 \mathrm{~cm}$.

\subsection{Optical characterizations}

The optical performances of the microfiber lasers were measured by the $\mu$-PL 
system (section 2.5). Although an array, which contains several microfibers, was buried in the PDMS matrix, only one microfiber was measured because of the high homogeneity of the in-array microfiber lasers. As illustrated in Fig. 4.3, the excitation beam was guided to be perpendicular to the microfiber and formed an angle of $45^{\circ}$ with the X-Y-Z stage which held the PDMS matrix. The emitted signal was collected with the objective (50X) from the top.

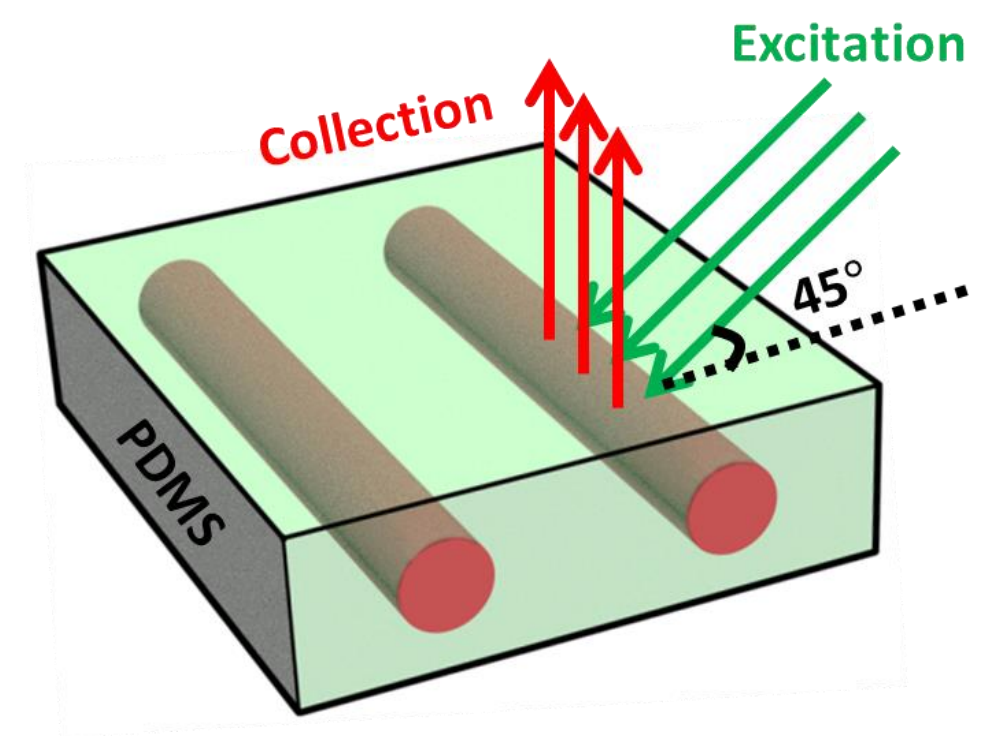

Figure 4.3. Excitation and collection of the microfiber laser.

The recorded photoluminescence (PL) spectra of the microfiber lasers are shown in Fig. 4.4 (a). Stimulated emission, represented by the bright optical image shown in the inset of Fig. 4.4 (b) and the sharp peaks in the spectrum, can be clearly observed. In order to further study the lasing mechanism, the two-dimensional WGM model is applied due to the circular cross-section of the microfiber (dashed line in Fig. 4.4 (b)), which is expected to sustain WGM lasing. It is known that the WGM resonances are characterized by factors including the size of the cavity, refractive indexes of the resonator and the surrounding medium, and the three mode numbers (section 1.3.1, Eq. 
(1.7)). ${ }^{[6]}$ Due to the high $Q$ factors, the fundamental modes are much easier to be observed in experiments than the higher order modes so the longitudinal mode number $m$ equals to the azimuthal mode number $l$ and the radial mode number $r$ is $1 .^{[6]}$ The doping of PMMA $\left(n_{P M M A}=1.50\right)$ in epoxy resin $\left(n_{\text {epoxy }}=1.41\right)$ during the fabrication results in the cavity refractive index of $1.47^{[45]}$ and as the PDMS matrix provides an environmental refractive index of 1.41, the calculated fundamental TM modes of the 33- $\mu \mathrm{m}$ microfiber laser fit closely to the experimental results (Fig. 4.4 (a)). The average error of the calculated $T M$ modes is $7.84 \%$ with respect to the free spectral range (FSR, $2.55 \mathrm{~nm}$ ), which is much lower than the error of the calculated $T E$ modes (35.49\%). In addition, the $Q$ factor, which is experimentally defined as the resonant wavelength over the full width half maximum, is measured to be around 7400 (inset of Fig. 4.4 (a)). Thus, the mechanism can be considered to be high-quality WGM lasing. Furthermore, single mode lasing was also observed in the microfiber laser with small diameter (upper spectrum of Fig. 4.4 (a)) because of the low $Q$ factor and large out-coupling efficiency. 

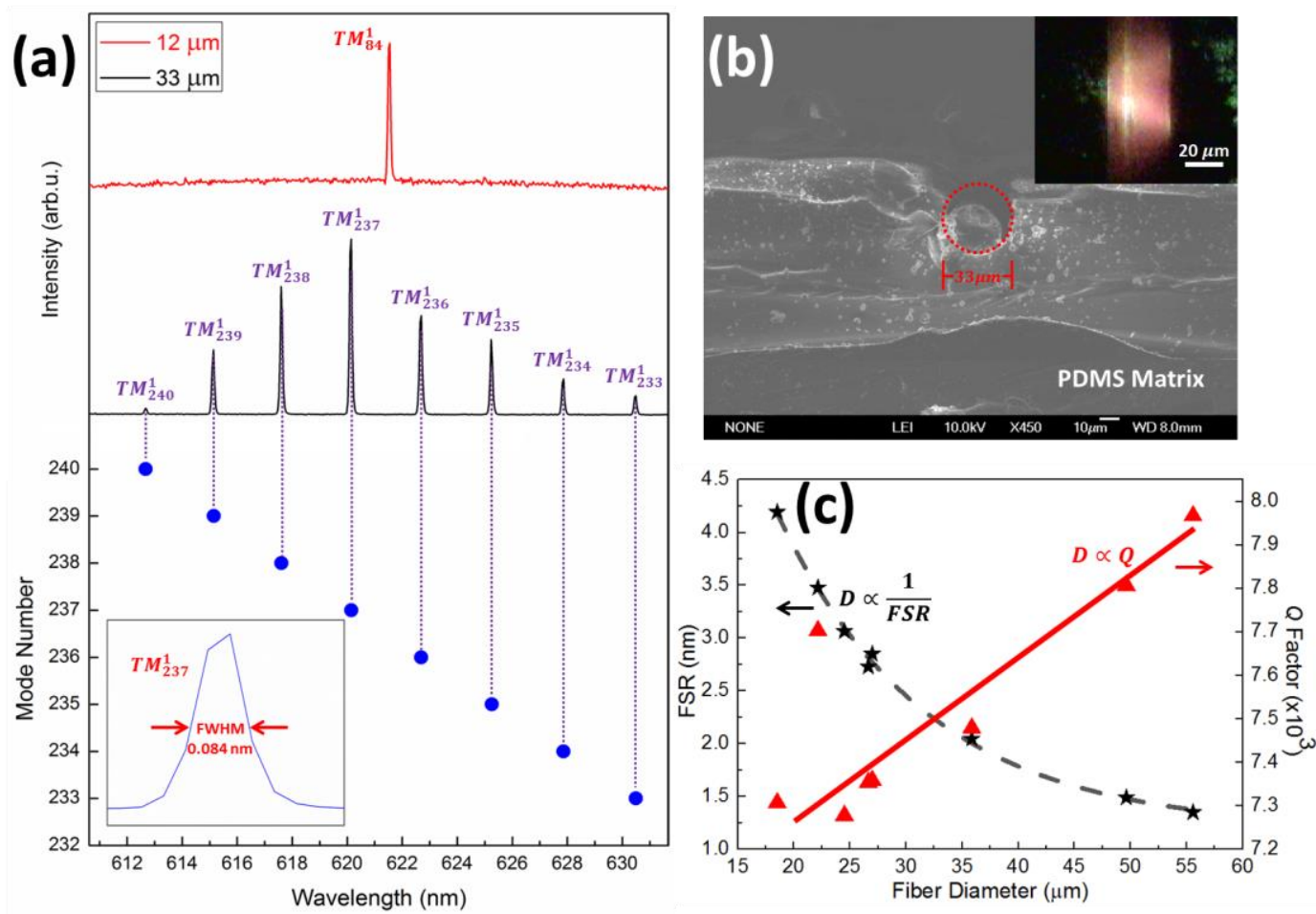

Figure 4.4. Optical performances of the microfiber lasers.

(a) Spectra of the active microfibers under pumping. Single mode lasing is achieved in the 12- $\mu m$ microfiber. All the modes are well-fitted with the WGM theory and the inset shows the enlarged spectrum of mode $T M_{237}^{1}$. (b) Scanning electron microscope (SEM) image of the cross-section of the microfiber buried in PDMS matrix. The red dashed line indicates the circular shape of the WGM plane and the inset shows the optical image of the 33- $\mu \mathrm{m}$ microfiber under excitation. (c) The dependence of free spectral range and quality factor on fiber diameters.

Theoretically, the FSR can be predicted by FSR $=\lambda^{2} / n \pi D$, which is inversely proportional to the diameter $(D)$ of the cavity (section 1.3.5). The experimental results and theoretical curve are fairly well-fitted in Fig. 4.4 (c), which further confirms the WGM lasing mechanism. Meanwhile, the $Q$ factors of the WGM cavity increase linearly with the increase of cavity size (Fig. 4.4 (c)). Indeed, although varying among microfibers with different diameters, the $Q$ factors remain in the magnitude of $10^{3}$, for which enhanced light-matter interactions and high-quality WGM lasing would be expected. 


\subsection{Mechanical tuning of the microfibers and the sensing applications}

Due to the elastic properties of both the PDMS matrix and the polymer microfiber lasers, the whole system (microfiber array buried in PDMS) can be utilized as a strain sensor or a force sensor by embedding the two ends of the PDMS matrix in a fixed and a movable holder (Fig. 4.5 (a)). When the PDMS matrix is extended, or the stage is moved, the cross-section of the microfiber, which is the main WGM plane, will be deformed and enlarged. As illustrated in Fig. 4.5 (b), the circular WGM plane becomes elliptical because of the external forces transferred by the matrix and therefore, the resonant condition is changed to:

$$
\left(n_{e f f}+\Delta n_{e f f}\right)\left(\pi \sqrt{2\left(a^{2}+b^{2}\right)}\right)=m(\lambda+\Delta \lambda)
$$

where $\mathrm{n}_{e f f}$ and $\Delta \mathrm{n}_{e f f}$ are the original effective index and the change of the effective index, respectively, $a$ is the half length of the major axis of the ellipse, $b$ is the half length of the minor axis of the ellipse, $\lambda$ is the original resonant wavelength and $\Delta \lambda$ is the shift of the resonant wavelength. Eq. (4.1) is based on the assumption that the elongation of the PDMS matrix is small, which does not influence the longitudinal mode number $m$ (or the wavelength shift is smaller than the free spectral range). After transformation, Eq. (4.1) can be rewritten as:

$$
\frac{\Delta \lambda}{\lambda}=\sqrt{\frac{a^{2}+b^{2}}{2 R^{2}}}-1+\sqrt{\frac{a^{2}+b^{2}}{2 R^{2}}} \frac{\Delta n_{e f f}}{n_{e f f}},
$$

where $R$ is the radius of the undeformed microfiber. It can be inferred from Eq. (4.2) that the shift of the resonant wavelength is an integrated result from both the change of the cavity perimeter and the refractive index variation. However, the two 
components compensate each other in this configuration, as the circumference-enlargement shifts the peaks towards the infrared region while the "diluted" refractive index due to the stretching of the PDMS matrix blueshifts the spectrum. ${ }^{[45,118]}$ Therefore, the dominant factor needs to be determined experimentally.

(a)

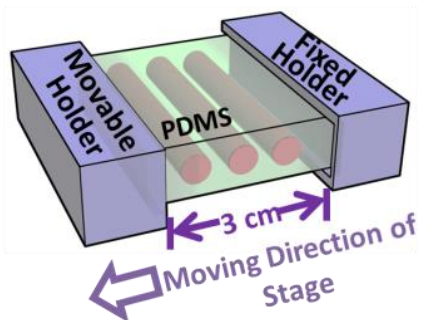

(b)

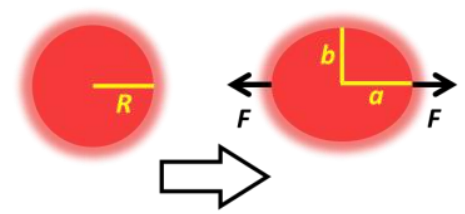

Figure 4.5. Schematic of the buried microfiber lasers as strain sensors.

(a) Illustration of the experimental setup for the strain sensors. (b) The cross-section deformation of the microfibers under the elongation of the PDMS matrix.

The experimental results of the tuned lasing peaks with respect to the elongation of the PDMS matrix are shown in Fig. 4.6 (a) and (b). The redshift of the modes can be seen clearly from the spectra, which is due to the dominant circumference-variation induced by the external forces. The results are understandable because the length-variation of the PDMS matrix is only $0.4 \%(0.12-\mathrm{mm}$ elongation for the 30-mm matrix), which does not influence the refractive index much and cannot overwhelm the effect brought by the circumference-enlargement. Therefore, it is reasonable to simplify Eq. (4.2) to:

$$
\frac{\Delta \lambda}{\lambda}=\sqrt{\frac{a^{2}+b^{2}}{2 R^{2}}}-1 .
$$

As the relative variation is the same for both the cross-sections of the microfibers and 
the PDMS matrix under the assumption of uniform elongation, $a$ and $b$ are calculated to be $16.56 \mu \mathrm{m}$ and $16.47 \mu \mathrm{m}$, respectively $(0.12-\mathrm{mm}$ increasing in the horizontal direction, or the moving direction of the stage, and $1.78-\mu \mathrm{m}$ decreasing in the vertical direction for the PDMS matrix). Substituting $a=16.56 \mu \mathrm{m}, \quad b=16.47 \mu \mathrm{m}$, $R=16.50 \mu \mathrm{m}$ and $\lambda=620.14 \mathrm{~nm}$ into Eq. (4.3) yields $\Delta \lambda=0.85 \mathrm{~nm}$, which is close to the experimental measurement shown in Fig. 4.6 (b) and further proves that the influence of the index-variation of the matrix is negligible. In addition, the strain sensitivity for this $33-\mu \mathrm{m}$ microfiber is calculated to be $0.22 \mathrm{pm} / \mu \varepsilon$.
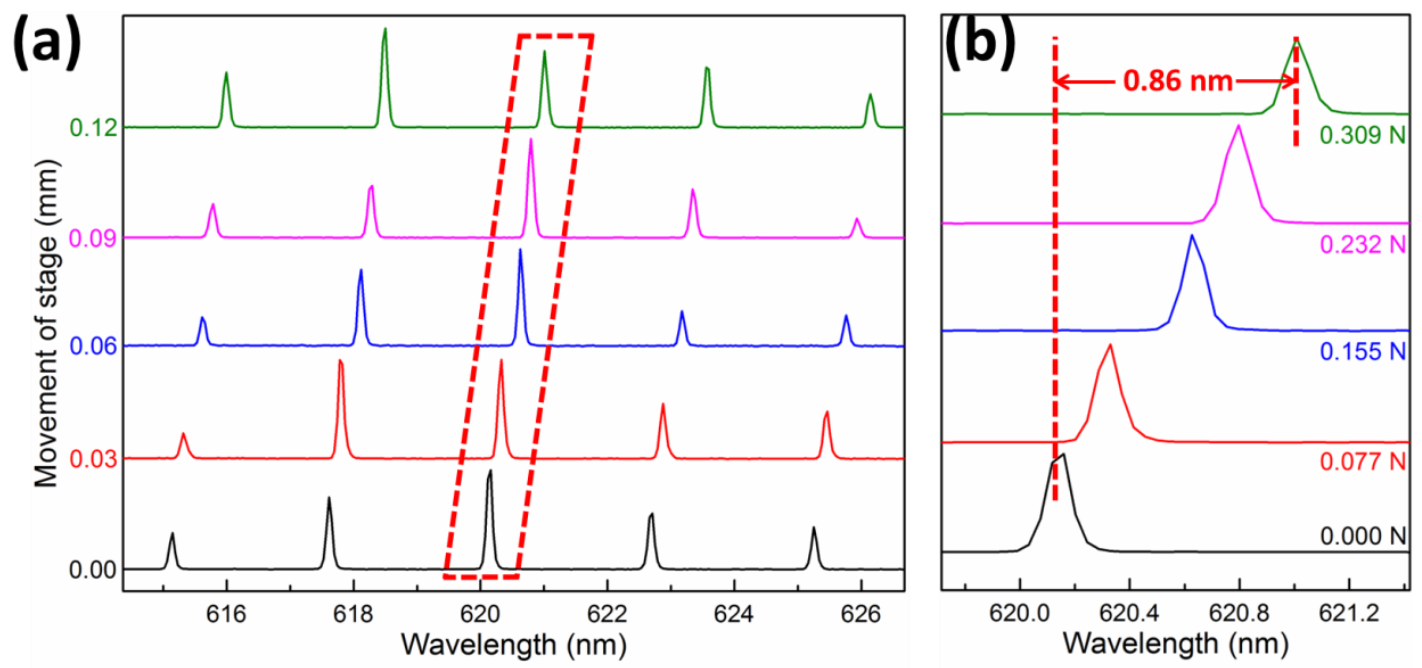

Figure 4.6. Spectra of the tuned lasing peaks with respect to the elongation of the PDMS matrix.

(a) Redshift of the modes with the movement of the stage. The diameter of the measured microfiber is $33 \mu \mathrm{m}$. (b) Enlarged spectrum of the highlighted part in (a) with the calculated external forces applied on the PDMS matrix.

The external forces applied to the PDMS matrix are derived and shown in Fig. 4.6 (b) by applying the linear Young's modulus model: ${ }^{[154]}$

$$
F=\frac{A E \Delta L}{L}
$$

where $A$ is the cross-sectional area of the applied force, $E$ is the Young's modulus of 
the material $\left(1.84 \times 10^{6} \mathrm{~Pa}\right.$ for PDMS, data obtained from the database of Sandia National Laboratories), $\Delta \mathrm{L}$ is the extension length and $L$ is the original length. The length, width and height of the PDMS matrix are measured to be $3 \mathrm{~cm}, 3 \mathrm{~cm}$ and 1.4 $\mathrm{mm}$, respectively. Therefore, with a maximum applied force of $0.309 \mathrm{~N}$, the lasing modes redshift $0.86 \mathrm{~nm}$ (Fig. 4.6 (b)), which results in the force sensitivity of 2.78 $\mathrm{nm} \cdot \mathrm{N}^{-1}$

In order to further investigate the relations between the intrinsic properties of the microfibers and the sensitivities, a comparative experiment was performed and the results are shown in Fig. 4.7. As represented by the blue curve, the sensitivity is proportional to the diameter of the microfiber when the PMMA to epoxy mass-ratio is fixed at 0.4 . This is probably because the circumference variation is larger for bigger fibers than for smaller ones under the same level of deformation, which leads to more obvious wavelength shift. On the other hand, when the fiber diameter is fixed at $45 \mu \mathrm{m}$, the sensitivity is inversely proportional to the PMMA to epoxy mass-ratio (red curve in Fig. 4.7), which is caused by the large proportion of PMMA in the cavity materials. As mentioned previously, the relative variation is assumed to be the same for both the cross-sections of the microfibers and the PDMS matrix at $m_{P M M A}: m_{\text {epoxy }}=0.4$. However, the assumption might be invalid for other ratios because the large amount of PMMA, which introduces high viscosity and rigidity to the microfiber, prevents the uniform deformation of the cross-sections with the PDMS matrix. In addition, it is worth noting that $m_{P M M A}: m_{\text {epoxy }}=0.4$ is the critical point for the microcavity to maintain the shape of a fiber and ratios lower than 0.4 will lead 
to the breaking of the fiber into microspheres or hemispheres. ${ }^{[4]}$ The highest sensitivity of $0.45 \mathrm{pm} / \mu \varepsilon$ in strain and $5.66 \mathrm{~nm} \cdot \mathrm{N}^{-1}$ in force, which is better than the WGM sensors based on PMMA balls, ${ }^{[153]}$ is supported by a $45-\mu \mathrm{m}$ microfiber with the PMMA to epoxy mass-ratio of 0.4 . Furthermore, the limit of detection, which is defined as the FWHM of the peak over the sensitivity, ${ }^{[15]}$ is calculated to be $1.76 \times$ $10^{-4} \varepsilon$ (strain) and $0.014 \mathrm{~N}$ (force) in this case. Although the sensing performances are not as good as the widely applied FBG strain sensors, ${ }^{[152]}$ the novel approach brought by the microfiber-PDMS system still holds large potential for improvement. Increasing the $Q$ factors by advanced processing techniques and materials, as well as simplifying the interrogation system all make the soft-approached WGM strain sensors more applicable in the future.

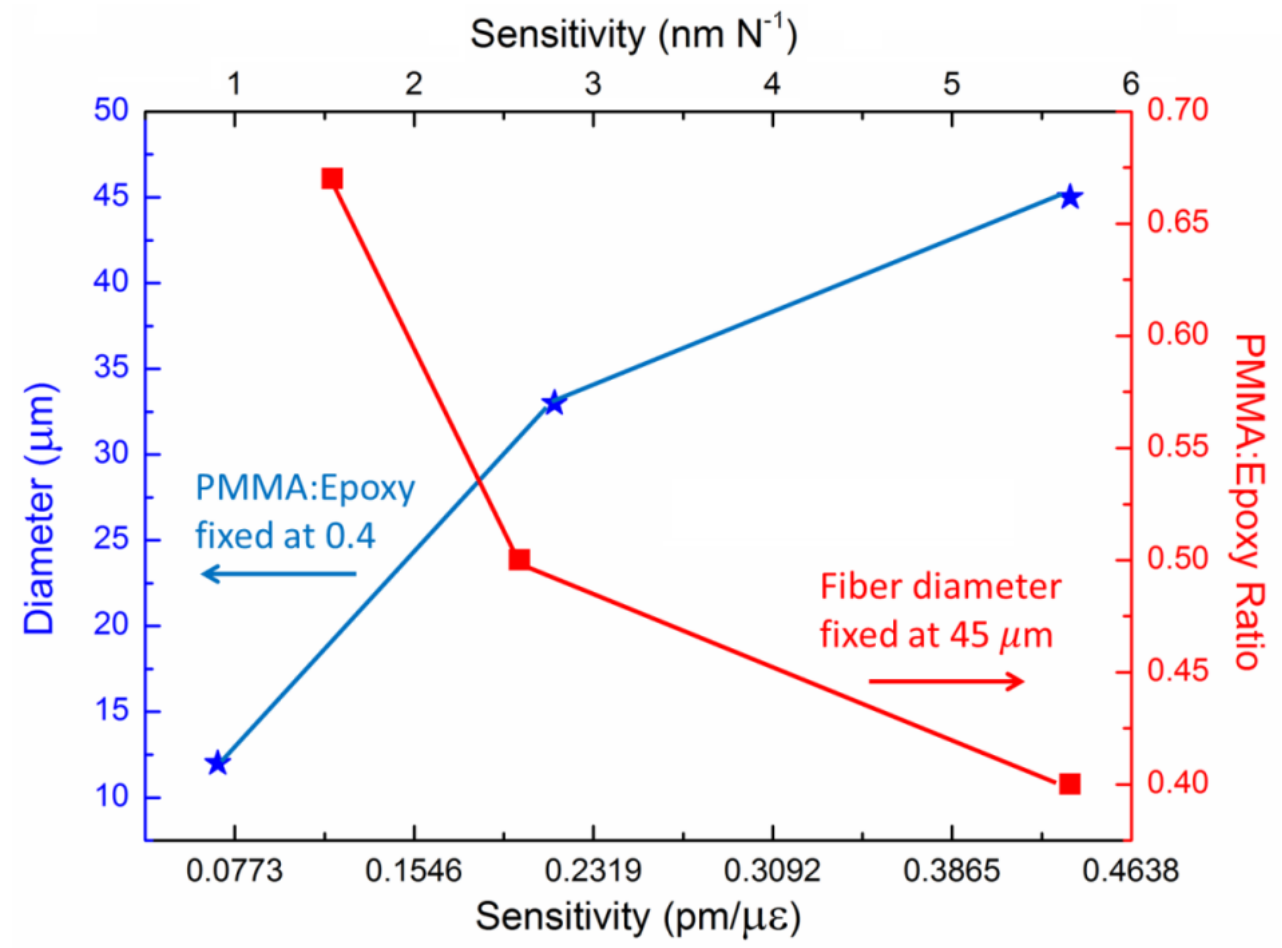

Figure 4.7. The relations between the fiber diameter, PMMA to epoxy mass ratio, and the sensitivity. 


\subsection{The advantages of the microfiber array sensors in PDMS}

The thermal-induced wavelength shift, which is introduced by the long-time excitation, is one of the most challenging issues in the sensing applications of the dye-doped lasers and dramatically decreases the reliability of the single-element-based sensors. ${ }^{[48]}$ In contrast, the microfiber arrays proposed in this work provide a practical method to solve this problem by alternatively exciting/scanning the microfiber lasers in the same PDMS matrix. The high homogeneity of the microfibers fabricated by the spiral drawing technique ensures the stabilization of the spectra in different microlasers. Indeed, comparing with the unwanted wavelength shift induced by long-time pumping of a single element in applications such as structural health monitoring, the sacrificed limit of detection or the non-uniformity of the fiber diameters in the array due to the unavoidable errors in the fabrication processes is acceptable.

In addition, the conventional WGM sensors are normally exposed to air without any protection, ${ }^{[145]}$ which may not only be affected by the contaminants attached on the surface of the resonators but also show various sensitivities when working under different environments such as the in-water and chemical vapor-surrounded situations. However, as the PDMS matrix provides fixed environmental index for the microfiber array buried inside, the proposed sensing configuration in this work is expected to exhibit consistent sensitivity, which is much more stable and reliable. Furthermore, the PDMS also protects the microfibers from photooxidation and contaminant attaching so the service lifetime of the sensors is extended. ${ }^{[45]}$ 
Comparing with the FBG strain sensors which provide limited sensing direction due to the configuration of the gratings, ${ }^{[155-157]}$ the WGM microfibers are multidirectional and multi-point effective due to the symmetric of the circular WGM planes and the unlimited cross-sections of the cylindrical geometry. It is also reported that the dye-doped polymer fibers are sensitive to the change of the refractive index and the force or strain in the axial direction. ${ }^{[45,158]}$ Besides, the non-contact signal collection mode enables the buried microfiber lasers to be utilized in some extreme cases such as the strong-electric-field and underwater environments where the traditional contact strain sensors cannot be conveniently used. Therefore, the spirally drawn polymer microfiber arrays buried in the PDMS matrix not only break the bottleneck of sensitivity limitation of the rigid WGM sensors but also show multifunctional, stable and reliable potentials compared to the FBG sensors and other WGM candidates based on a single element.

\subsection{Summary and outlook}

In summary, organic dye-doped polymer microfiber arrays buried in PDMS matrix with controlled fiber-size and position is achieved via the spiral drawing technique. The high-quality WGM lasing, which is supported by the circular cross-sections of the microfibers, is observed and tuned via the mechanical force-induced elongation of the PDMS matrix. Detailed studies reveal that the wavelength shift is dominated by the enlargement of the cavity-circumference under deformation. The buried microfiber arrays can be further utilized as strain and force 
sensors with the sensitivity of $0.45 \mathrm{pm} / \mu \varepsilon$ (strain), $5.66 \mathrm{~nm} \cdot \mathrm{N}^{-1}$ (force), and limit of detection down to $1.76 \times 10^{-4} \varepsilon$ (strain), $0.014 \mathrm{~N}$ (force). Besides, with the homogeneous array structure and the outer protection of the PDMS matrix, the proposed soft-approached strain sensors are stable, reliable and multifunctional, making them competitive compared to other sensors. This work not only proposes a novel, efficient and controllable fabrication method for the microfiber lasers, but also broadens the applications of the soft-approached WGM microcavities and breaks the bottleneck of sensitivity limitation of the rigid WGM sensors. The demonstrated strain sensors are expected to be applicable in several fields, such as structural health monitoring, multi-point perturbation detection, and even some applications in extreme environments.

The improvement of the strain and force sensors based on the microfiber arrays can be implemented in two aspects. The first is to better control the spiral drawing approach or replace it with a more precise technique to realize smaller standard deviations of the diameters of the in-array microfibers. The enhanced homogeneity will further increase the reliability of the devices and lower the limit of detection to its theoretical value. The second is to simplify the interrogation system to further enhance the practicality of the devices. Indeed, with the development of the excitation technique (CW pumping or even electrical pumping of the microfibers in the future), the issue of complexity and cost of the interrogation system can be resolved. 


\section{Reconfigurable Tuning of Liquid Whispering Gallery Mode Microlasers via Surface Tension}

\subsection{Introduction and motivation}

As introduced in Chapter 1, WGM microcavities and microlasers have aroused great research interests not only due to their high $Q$ factors, small mode volumes, and capability to enhance light-matter interactions, but also because of the abundant applications in the fields of optoelectronic devices, quantum information processing, nonlinear optics, materials studies, biological and chemical sensing, etc. ${ }^{[6]}$ Among all the demonstrated works, those that engineer WGM microcavities and microlasers in microfluidic channels or liquid environments are significant owing to their potential compatibility and applications in biology and medical sciences. ${ }^{[159-162]}$ However, the bio-compatible liquid environments require the WGM microcavities and microlasers to be flexible, stable, reconfigurable, and highly tunable, which are hard to achieve in conventional solid-state architectures due to the rigid nature of the semiconductor and glassy materials. ${ }^{[6]}$ In contrast, soft materials like polymers and liquid crystals are elastic and flexible, which are the key properties for tunable microlasers in liquids. ${ }^{[46,}$ ${ }^{163-165]}$ Although several tuning works have been reported based on the modification of gain medium, effective index and cavity size of the soft-approached WGM microlasers (see Chapter 1.5), drawbacks like small shifting range of wavelengths, short cavity lifetimes, complex external conditions (electric/magnetic fields), as well as unidirectional tuning still exist. ${ }^{[15]}$ In addition, reconfigurable tuning of the soft 
WGM cavities in liquid remains challenging. Therefore, a novel quasi-disk floating microlaser is introduced in this chapter via the inkjet printing technique by the microplotter. High-quality WGM lasing is observed and further tuned (up to $10 \mathrm{~nm}$ ) by employing the surfactants, which can modify the surface tension of the liquid environment and lead to the reconfigurable transformation of the cavity shape. Meanwhile, sensing of the polar chemicals exemplified by ethanol is achieved via a quasi-disk microlaser with the FSR sensitivity of $19.85 \mathrm{THz} /\left(\mathrm{mol} \bullet \mathrm{mL}^{-1}\right)$. This work not only demonstrates a novel and reconfigurably tuned WGM microlaser in the liquid environment, but also provides an insight into the potential applications of flexible and soft-approached WGM microcavities in microfluidics and bio/chemical sensing.

\subsection{Quasi-disk floating microlaser fabricated by inkjet printing}

The host solution for the quasi-disk floating microlaser was made by $1.0 \mathrm{mg}$ of Rhodamine 6G (R6G, from Sigma-Aldrich), which was dissolved in $0.1 \mathrm{~mL}$ of dichloromethane (purity: $99.7 \%$ ), and $400 \mathrm{mg}$ of epoxy resin (Araldite 506, from Sigma-Aldrich). The R6G was selected as the gain medium for lasing and the epoxy resin was chosen because of its transparency, optical and thermal stability, elasticity, as well as flexibility in doping, which make it an ideal host material for soft-approached microcavities. ${ }^{[28,45,47,118]}$ After ultrasonic vibrating the mixture for 30 minutes, the host solution was ready for cavity-fabrication. As illustrated in Fig. 5.1 (a), after dipping the microdispenser of the microplotter (section 2.1) into a 
droplet of the host solution, some of the dye-doped polymers were hung on the tip of the dispenser. Then the microdispenser was controlled to gently touch the surface of the water, which was mixed with soap water to modify the surface tension of the liquid environment (Fig. 5.1 (b)). Finally, the microdispenser was lifted up slowly and the polymers hanging on the tip was released on the surface of the water and self-assembled to be a circular quasi-disk floating microlaser to minimize the surface energy (Fig. 5.1 (c) and (d)). The initial size of the quasi-disk microlaser could be controlled by the amount of polymers hung on the tip, which is related to the size of the microdispenser and the immersion depth of the tip into the host solution. The soap water was a mixture of $8 \mu \mathrm{L}$ of Mama Lemon (Lion Corporation, Japan) with $25 \mathrm{~mL}$ of water and the liquid environment for the quasi-disk microlaser was prepared by adding $0.2 \mathrm{~mL}$ of soap water into $2.5 \mathrm{~mL}$ of water.
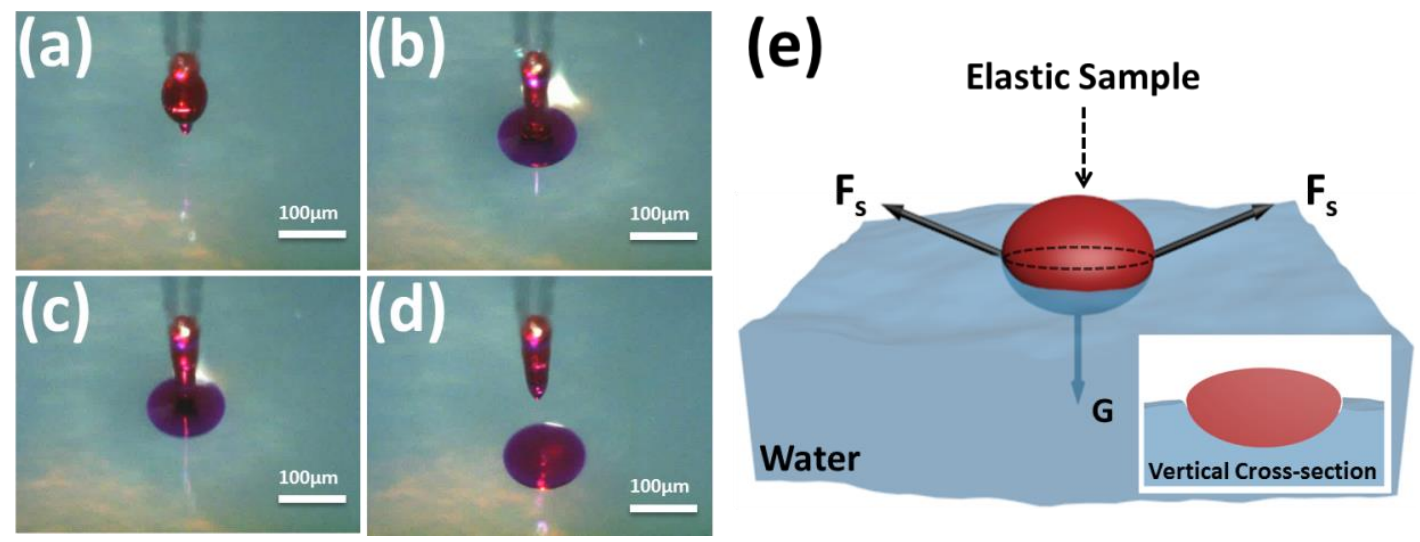

Figure 5.1. Inkjet printing of the quasi-disk floating microlaser.

(a) Polymers hung outside the tip of the microdispenser. (b) Dipping the tip into the liquid environment. (c) Slowly lifting up the microdispenser. (d) A self-assembled quasi-disk floating microlaser. (e) The schematic and force analysis of the inkjet-printed microlaser. The inset shows the vertical cross-section of the quasi-disk floating microlaser. ${ }^{[15]}$

The schematic and force analysis of the inkjet-printed quasi-disk microlaser is 
shown in Fig. 5.1 (e). Similar to the small objects (coins, paper clips, water striders, etc.) that can float on the surface of water, the quasi-disk microlaser was supported by the large surface tension of water molecules instead of the buoyancy due to the relatively large material density $\left(1.168 \mathrm{~g} / \mathrm{cm}^{3}\right.$, data obtained from Sigma-Aldrich). The forces provided by the surface tension are in equilibrium with the force of gravity and the vertical cross-section of the microlaser is expected to be irregular because of the different surface tensions at the cavity/water and cavity/air interfaces (inset of Fig. 5.1 (e)). However, the horizontal cross-section, which is the main lasing plane and marked by the dashed line in Fig. 5.1 (e), is circular due to the trend of minimizing the surface energy in liquid materials.

\subsection{Whispering gallery mode lasing characterization}

The optical performances of the quasi-disk microlasers were measured by the $\mu$-PL system (section 2.5). The excitation beam was guided to the sample with an angle of $45^{\circ}$ to the surface of the water. As illustrated in Fig. 5.2 (a), the evolution from broadband emission to lasing took place with the increase of the pumping intensities from $0.4 \mu \mathrm{J}$ to $1.9 \mu \mathrm{J}$. The circular horizontal cross-section, which can be seen clearly from the optical images in Fig. 5.2 (c) and (d), together with the bright ring near the boundary under excitation, suggest WGM lasing. Due to the single set of modes in the spectrum, 2D WGM model for fundamental modes is applied to verify the assumption. After substituting $n_{\text {cavity }}=1.41, \quad n_{\text {environment }}=1.33$ and $D_{\text {cavity }}=135 \mu \mathrm{m}$ into Eq. (1.7) and Eq. (1.36), the calculated FSR is $0.61 \mathrm{~nm}$, which 
is very close to the measured value from the spectrum (Fig. 5.2 (b)). Meanwhile, the modes can be well-fitted with the theoretical transverse magnetic modes, which are assigned in Fig. 5.2 (b). Thus, the lasing mechanism for the floating quasi-disk microlaser is proved to be WGM lasing.
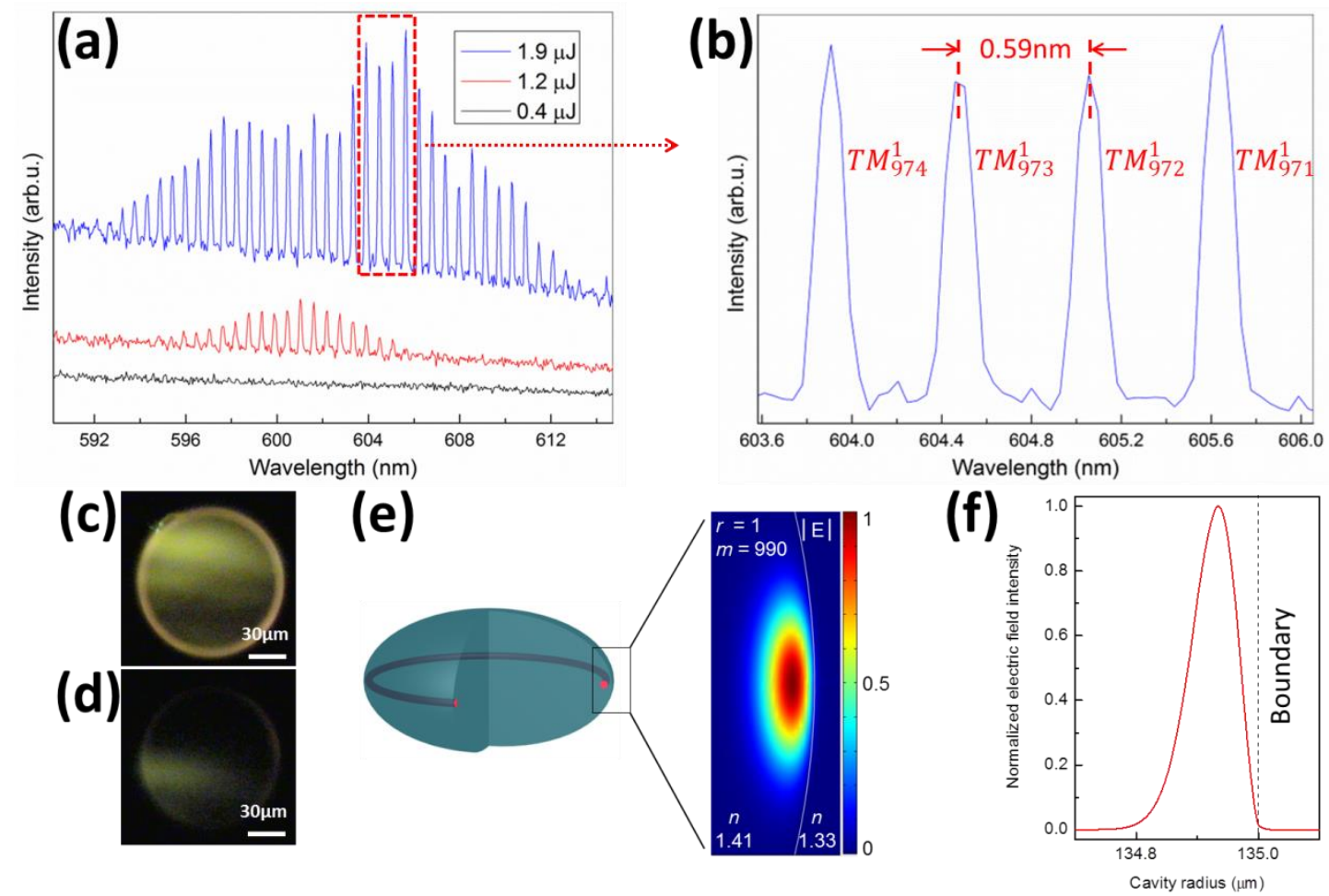

Figure 5.2. The WGM lasing and characterizations of the quasi-disk microlaser.

(a) The evolution from broadband emission to lasing with the increase of the pumping intensities. (b) Spectrum of the selected region in (a) and mode assignment. (c) Optical image of the quasi-disk microlaser above threshold. (d) Optical image of the quasi-disk microlaser below threshold. (e) Simulation results of a fundamental mode (resonant wavelength: $604.5 \mathrm{~nm}$ ) in the equatorial plane of the quasi-disk microlaser. The parameters applied in the simulation are the same as the experimental data. (f) Normalized electric field intensities of the fundamental mode near the boundary of the microlaser (represented by the dashed line). ${ }^{[15]}$

In addition, the numerical simulations were implemented in order to understand the field distribution and optical confinement inside the microlaser. The selected parameters (cavity size, refractive indexes, and resonant wavelength) in the simulation 
are set to the ones in the experiment so the results by COMSOL Multiphysics with the finite-element method $^{[166]}$ illustrate the real picture inside the quasi-disk microlaser. As exemplified by a fundamental mode (resonant wavelength: $604.5 \mathrm{~nm}$ ) in the equatorial plane of the microlaser (Fig. 5.2 (e)), the electric fields are mostly distributed near the boundary of the cavity so strong optical confinement of the mode and enhanced light-matter interactions take place at the equator, which results in the WGM lasing as well as the bright ring in the optical images. The further calculation of the electric field intensities shown in Fig. 5.2 (f) reveals that around $99 \%$ of the energy is confined inside the cavity while only $1 \%$ exponentially decays outside the boundary, which is referred as the evanescent waves of the quasi-disk microlaser.

\subsection{Tuning mechanism of the floating microlasers}

It is found that the flexible and elastic epoxy-resin-based quasi-disk microlasers are highly tunable and sensitive to the liquid environment. As shown in the optical images in Fig. 5.3, the whole water surface was covered with the dye-doped epoxy resin solution when the environmental material was pure water (Fig. 5.3 (a) and (b)). However, the same amount of epoxy resin would shrink to several circular quasi-disk microlasers by adding 15 drops of soap water into the pure water initially (Fig. 5.3 (c)) and the sizes of all the microlasers were further decreased to the level of micrometer with the increase of the soap-water concentration (Fig. 5.3 (d) and the inset). It is important to note that at a critical point of the soap-water concentration, the sizes of the microlasers would reach their limit as the intrinsic intermolecular forces of the 
epoxy resin would protect the microlaser from further shrinking.

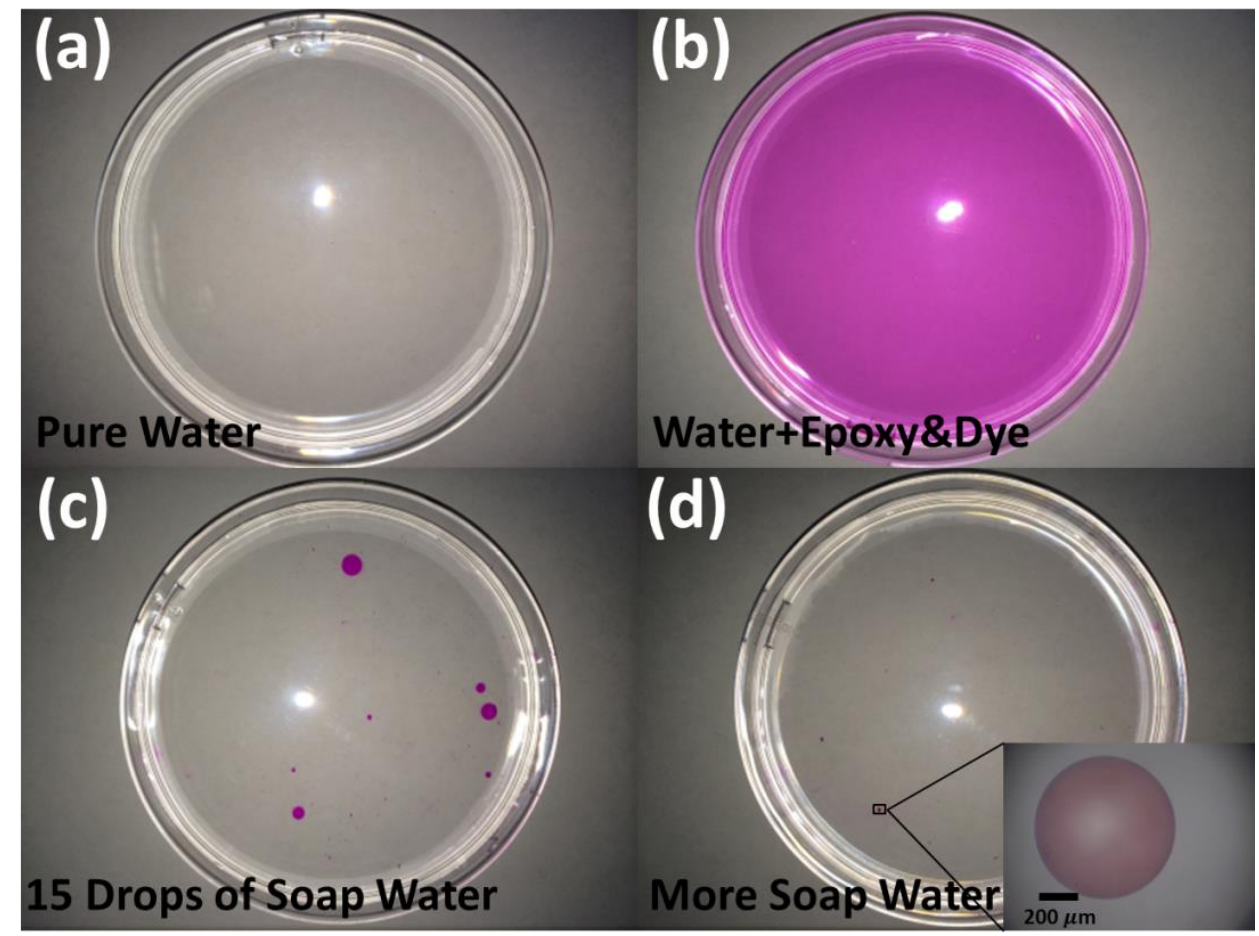

Figure 5.3. The shrinkage of the quasi-disk microlasers via the modification of the liquid environment.

(a) Pure water. (b) Pure water with the dye-doped epoxy resin covered on the top. (c) Floating quasi-disk microlasers by initially adding 15 drops of soap water into the pure water. (d) Decreased sizes of the microlasers with the increase of the soap-water concentration.

The interesting phenomena can be explained by the force analyses of the floating quasi-disk microlasers and the theory of surface tension. ${ }^{[167,168]}$ As illustrated in Fig. 5.4 (a), the surface tension of water is due to the unbalanced intermolecular forces of the water molecules at the surface, which "pull" the surface molecules inward and form a curved boundary to minimize the surface energy. However, the existence of polar molecules, such as the surfactants in the soap water, can attract the water molecules to reduce the intermolecular forces between the water molecules because of polarity, ${ }^{[168]}$ which induces the decrease of the surface tension and the curvature of the 
water/air boundary.

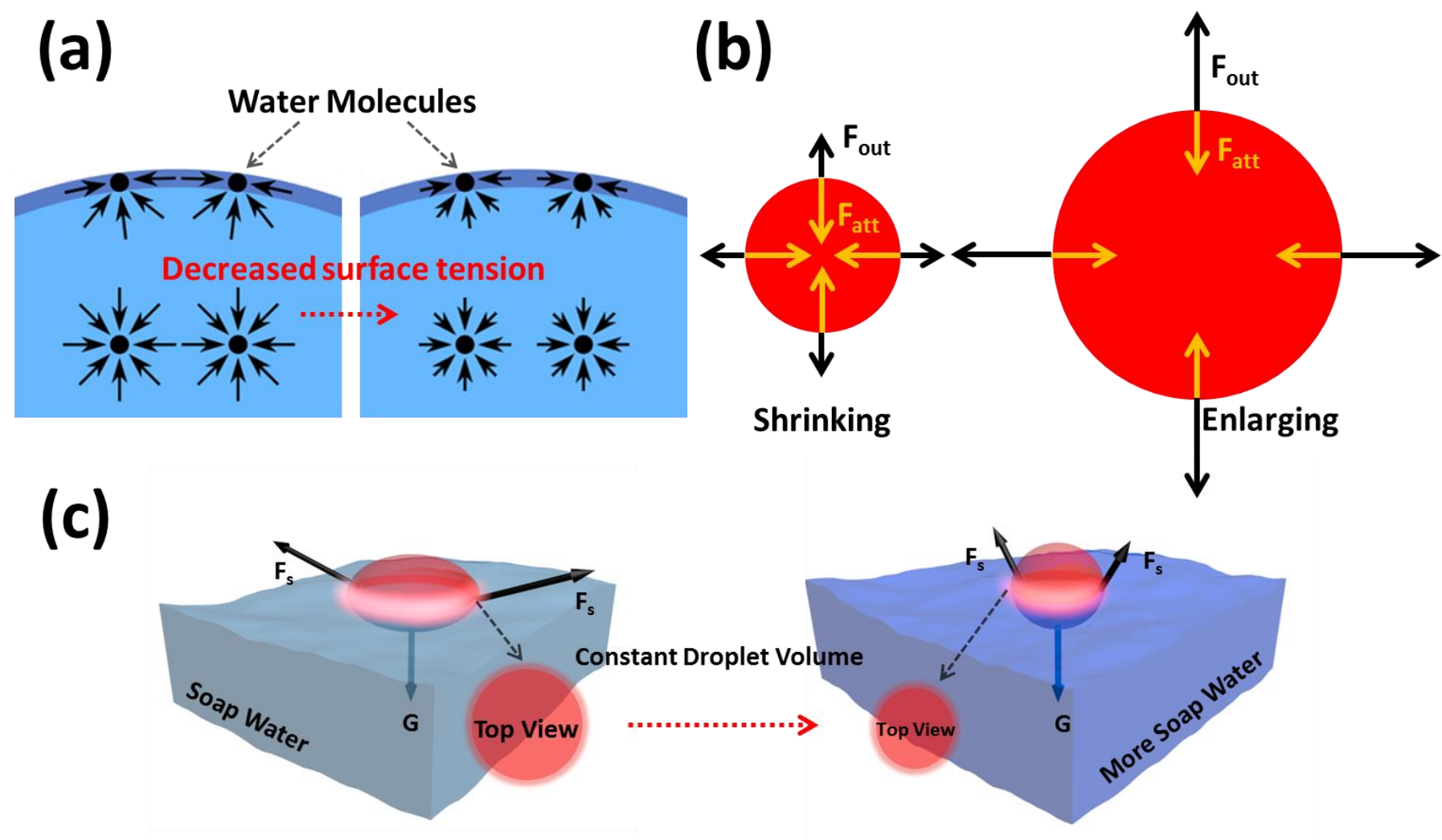

Figure 5.4. Illustration of the surface-tension-induced deformation of the floating quasi-disk microlaser.

(a) The decreasing of surface tension via adding polar surfactants in pure water. (b) Force analyses of the horizontal cross-section of a quasi-disk microlaser. (c) Three-dimensional illustration of the cavity deformation process. ${ }^{[15]}$

From the view of the quasi-disk microlaser, the equilibrium system of the forces provided by the surface tension $\left(F_{S}\right)$ and the force of gravity $(G)$ is demonstrated in Fig. 5.1 (e). Seen from the force analyses of the horizontal cross-section in Fig. 5.4 (b), the $F_{\text {out }}$, which is the horizontal component of $F_{S}$, is the same as the intrinsic intermolecular forces of the epoxy resin $\left(F_{\text {att }}\right)$ at equilibrium. However, if $F_{\text {out }}$ becomes smaller due to the decreased $F_{S}$ caused by the surfactants, the equilibrium is broken and the relatively large $F_{\text {att }}$ will shrink the cross-section to a new equilibrium where the new $F_{a t t}$, which is smaller than the old one because the 
attraction forces of the cavity molecules are decreased with the condensation of the cross-section, equals to the decreased $F_{\text {out }}$. Similarly, the WGM plane will be enlarged when $F_{\text {out }}$ is larger than $F_{\text {att }}$ until a new equilibrium is established. It is interesting to note that the large surface tension of water, or the huge $F_{\text {out }}$, will spread the dye-doped epoxy resin all over the water surface as a thin film without surfactants (Fig. 5.3 (b)) while the limitation of the intermolecular distance will prevent the cross-section from unlimited shrinkage (Fig. 5.3 (d)). The three-dimensional illustration of the cavity deformation shown in Fig. 5.4 (c) reveals that although the WGM plane is shrunk with the increase of the soap water concentration, the volume of the microlaser remains constant, which is due to the simultaneously enlargement of the vertical cross-section.
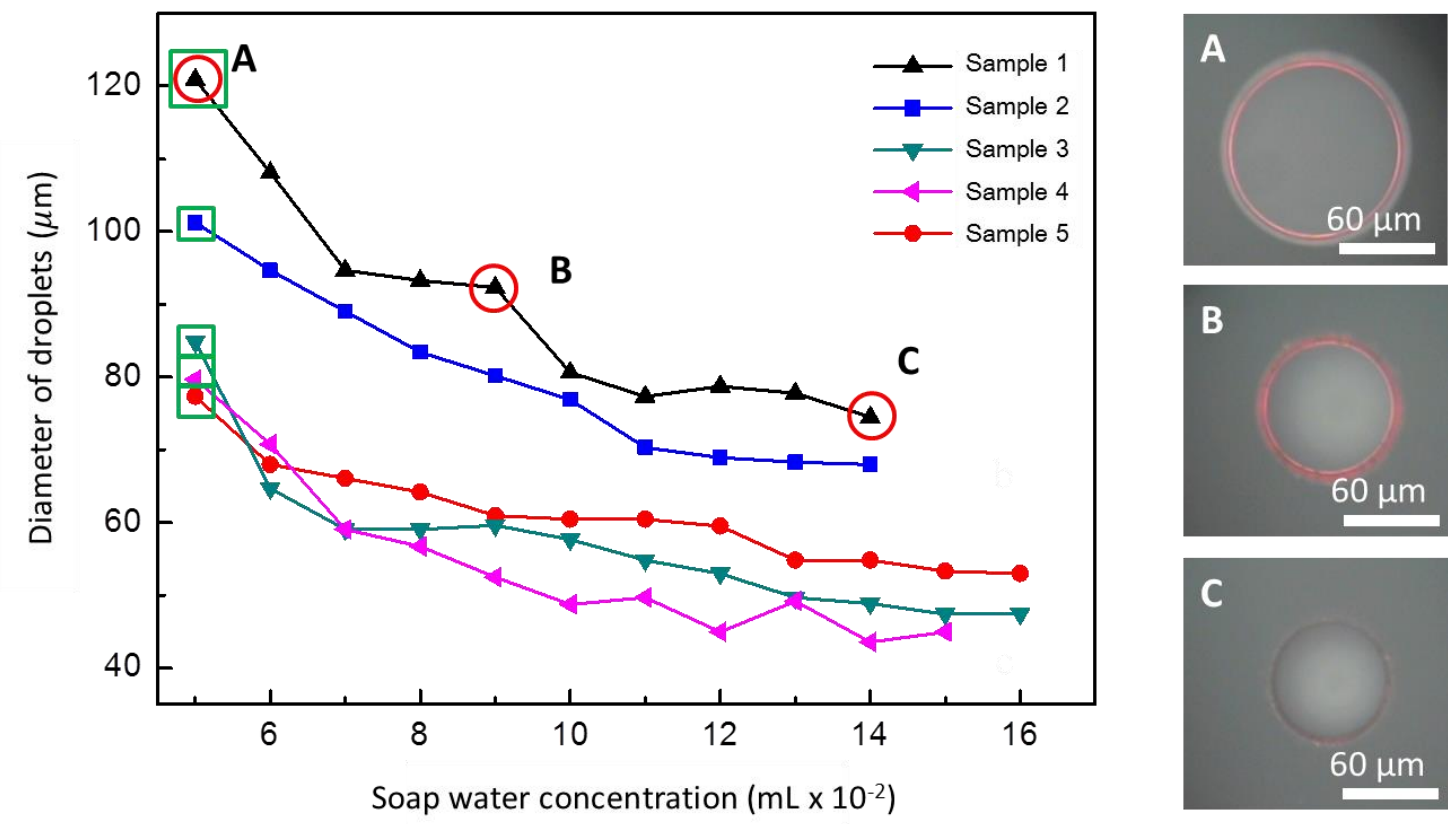

Figure 5.5. The shrinkage of the horizontal cross-sections of the floating quasi-disk microlasers with various initial sizes.

The optical images $A, B$, and $C$ correspond to the highlighted points in sample 1 and the green rectangles represent the initial diameters of the five samples. ${ }^{[15]}$ 
Fig. 5.5 reveals the experimental results of the effect of deformation of the floating quasi-disk microlasers with different initial diameters. All the tested samples are fabricated with the same initial soap water concentration $(\sim 8 \%)$ and show continuous decrease of diameters of the horizontal cross-sections with the increase of the soap water concentration. Clear shrinkage can be observed from the optical images $\mathrm{A}, \mathrm{B}$ and $\mathrm{C}$, which reveal the deformation processes of sample 1. Further measurements show that an average of $40 \%$ decrease in diameter can be achieved, which may be used to tune the wavelength of the floating quasi-disk microlasers.

\subsection{Reconfigurable wavelength tuning of the microlasers through environmental surface tension manipulation}

In order to examine the wavelength-tuning performances of the floating quasi-disk microlasers, a microfluidic system was made in the laboratory with a small container and several capillary tubes (Fig. 5.6). The sample is automatically located at the center of the liquid environment due to the surface-tension-induced large curvature of the liquid surface in a small container, which can stabilize the sample and

minimize the effect of Brownian motion of the water molecules on the spectra. ${ }^{[169]}$ Meanwhile, the tube $\mathrm{X}$ and $\mathrm{Y}$ are utilized together to control the concentration of the soap water in the liquid environment and the drain tube is used to keep the volume of the liquid in the container constant, which helps to maintain the excitation position and ensure the resulting spectra to be comparable. 


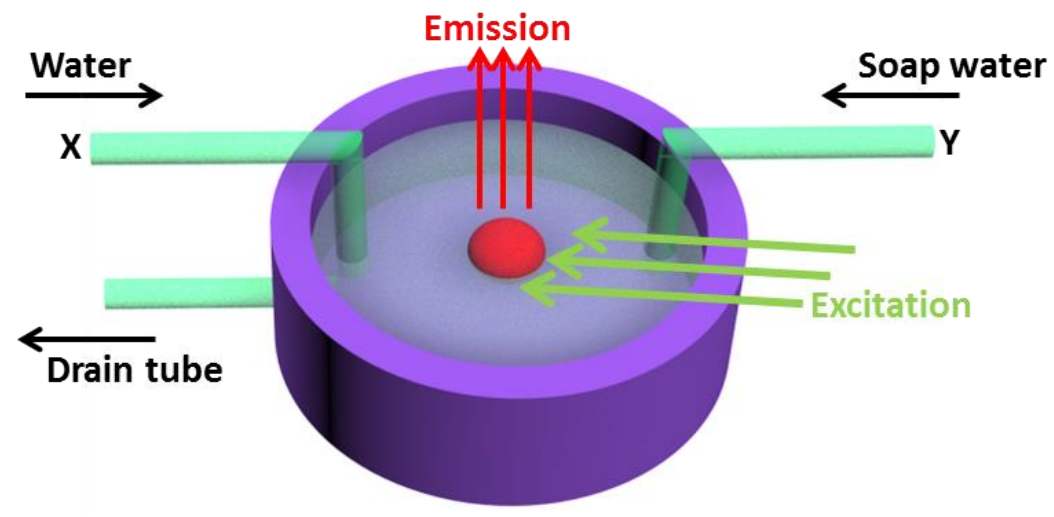

Figure 5.6. The microfluidic system for the measurements of wavelength tuning. ${ }^{[15]}$

The measured wavelength tuning is shown in Fig. 5.7 (a). From the spectra, it can be concluded that with the diameter of the quasi-disk microlaser decreases from $145 \mu \mathrm{m}$ to $105 \mu \mathrm{m}$, the lasing envelope shifts around $10 \mathrm{~nm}$ (from $\sim 603 \mathrm{~nm}$ to $\sim 593$ $\mathrm{nm})$, which is larger than the reported works. ${ }^{[45,98]}$ It is worth noting that the FSR, which is measured and marked in each spectrum, changes with the shift of the peaks following the WGM theory. Therefore, the FSR, as well as the bright rings in the optical images shown in Fig. 5.7 (b), prove the consistent high-quality WGM lasing through the deformation processes.

In the previously reported WGM microcavities and microlasers, the large ones normally hold higher $Q$ factors than the small ones because of the low optical loss. ${ }^{[53]}$ However, the measured $Q$ factors for the $145 \mu \mathrm{m}, 115 \mu \mathrm{m}$ and $105 \mu \mathrm{m}$ floating quasi-disk microlasers in Fig. 5.7 (a) are 4000, 5300 and 5800, respectively. Indeed, during the shrinkage process, the quasi-disk microlasers become thicker due to the enhanced curvature of the vertical cross-sections, which enables the deformed microlaser with small WGM plane to overlap more with the mode profile than the original ones with large horizontal cross-sections. Therefore, the optical loss for the 
shrunk quasi-disk microlaser is smaller, which induces larger $Q$ factors.

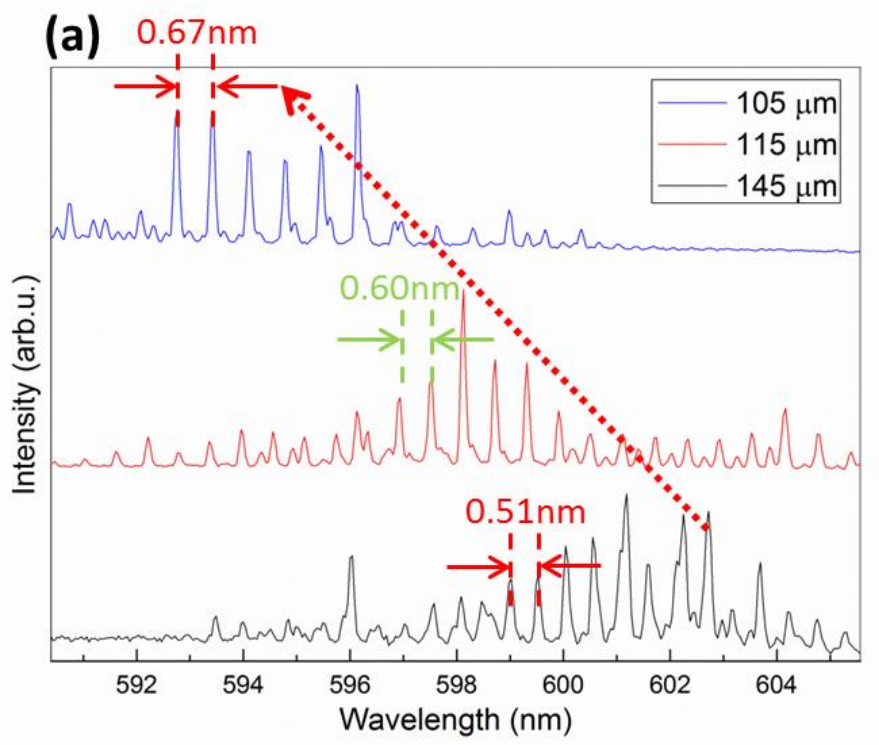

(b)
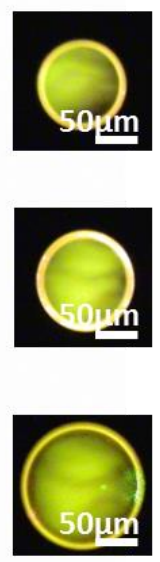

Figure 5.7. Blueshift of the lasing envelope with the decrease of the cavity size. (a) Measured lasing spectra during the shrinking processes of the floating quasi-disk microlaser. (b) Optical images of the excited microlaser with respect to the spectra. [15]

The blueshift of the lasing peaks cannot be derived directly from the asymptotic formulae introduced in section 1.3.1 as the tuning range is larger than the FSR, which means the mode numbers of the peaks vary in this case. Therefore, the analyses of the phenomenon should be based on the output power $P(D, \lambda)$, which is proportional to the out-coupling efficiency $\beta(D, \lambda)$, the mode volume of the cavity $V(D, \lambda)$ and the gain profile $g(\lambda):{ }^{[46,170,171]}$

$$
\begin{gathered}
\beta=\frac{Q_{r a d}^{-1}}{Q_{a b s}^{-1}+Q_{r a d}^{-1}}, \quad(5.1) \\
P(D, \lambda) \propto \beta(D, \lambda) \cdot \frac{V(D, \lambda)}{\lambda^{3}} \cdot g(\lambda),
\end{gathered}
$$

where $Q_{r a d}$ and $Q_{a b s}$ are the radiative quality factor and the absorptive quality factor, respectively. With the decrease of the cavity size, the radiative quality factor drops 
due to the weak confinement of the light in the small resonator and thus, the out-coupling efficiency is increased (Eq. (5.1)). On the other hand, large cavities can sustain longer wavelengths than the small ones for the same out-coupling efficiency so, as demonstrated in Fig. 5.8 (a), the overall performance of the out-coupling efficiency should be blueshifted with the shrinkage of the cavity size. Besides, because small WGM resonators always hold smaller mode volumes than the large ones, ${ }^{[172]}$ the term $\frac{V(D, \lambda)}{\lambda^{3}}$ also blueshifts when the cavity size is decreased (Fig. 5.8 (b)). In addition, the gain profile does not change with the cavity deformation. Therefore, as an overall result of the out-coupling efficiency, the mode volume and the gain profile (Eq. (5.2)), the output power of the microlaser is shifted to shorter wavelengths with the decrease of the diameter, which leads to the blueshifted lasing peaks.
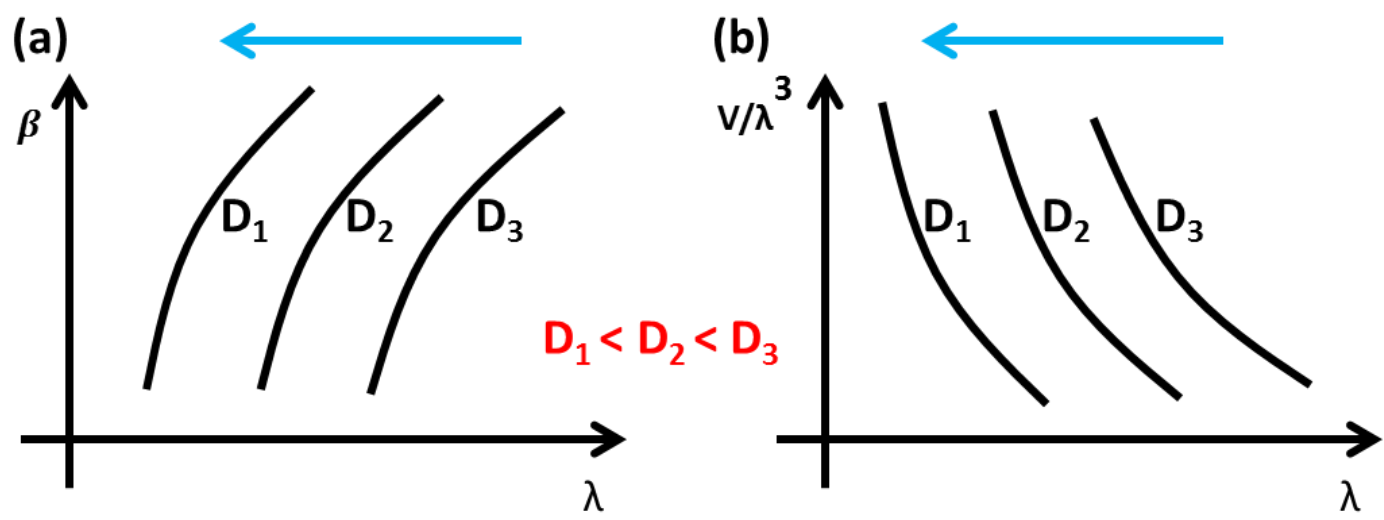

Figure 5.8. Analyses of the underlying physics that cause the blueshift of the lasing peaks. $D$ refers to the diameter of the microresonator.

(a) The out-coupling efficiency blueshifts with the decrease of the cavity size. (b) Mode volume blueshifts with the decrease of the cavity size.

It is interesting to note that the deformations of the floating quasi-disk microlasers, as well as the tuning of the lasing peaks, are reversible. As illustrated in 
Fig. 5.9 (b), with the surfactant concentration firstly increased and then decreased, the diameter of the microlaser was initially changed from $135 \mu \mathrm{m}$ to $105 \mu \mathrm{m}$ and finally tuned back to $130 \mu \mathrm{m}$. Correspondingly, the lasing peaks are blueshifted around 10 $\mathrm{nm}$ first and then redshifted $9 \mathrm{~nm}$, which show the reconfigurable tuning ability of the floating quasi-disk microlasers (Fig. 5.9 (a)). In addition, the proposed microlasers are stable under ambient conditions as revealed by the comparative experiments which show the undeformed cavity geometry for up to 4 hours after fabrication (without the modification of the surfactant concentration). Therefore, the bi-directionally tunable, reconfigurable and stable floating quasi-disk microlasers are promising in the fields of microfluidic sensing and bio/chemical sensing.

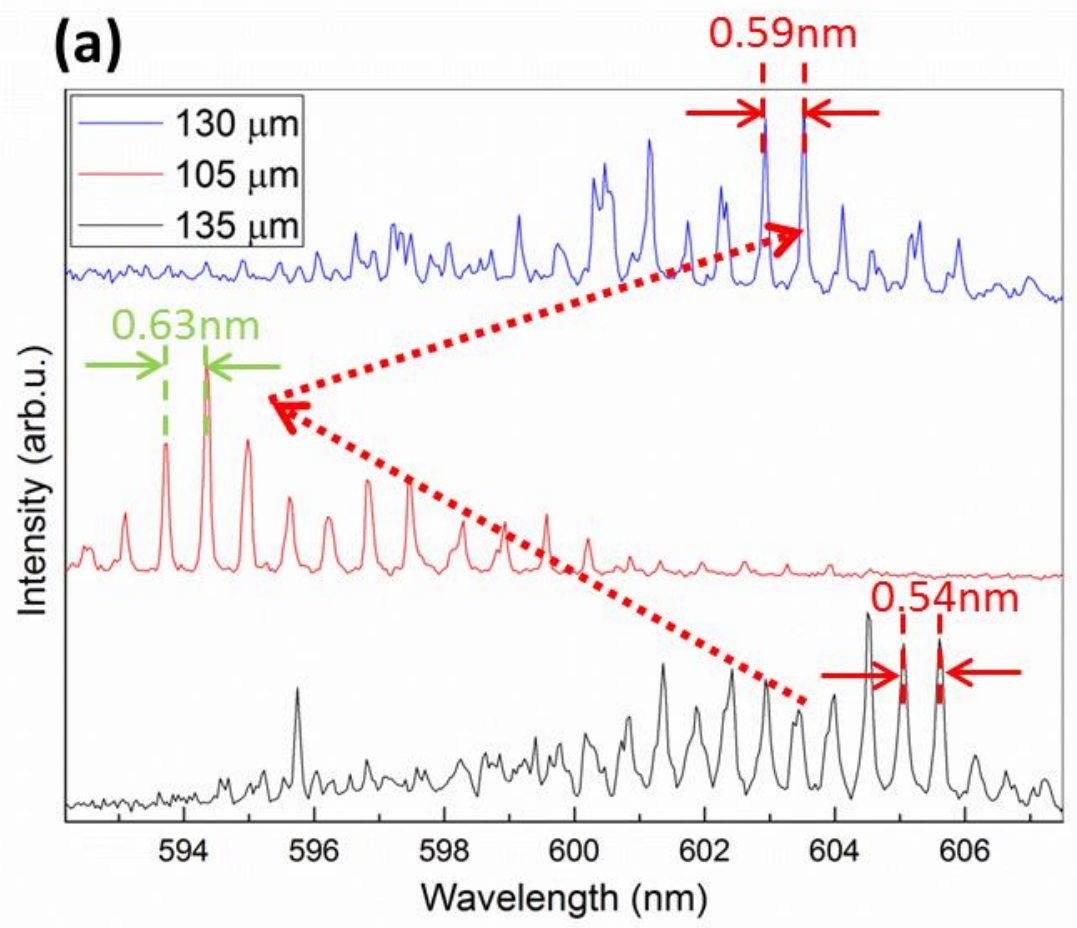

(b)
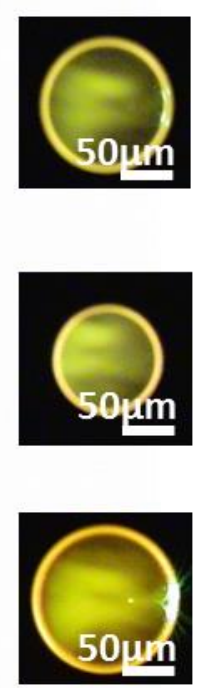

Figure 5.9. Reconfigurable tuning of the floating quasi-disk microlaser. (a) Measured lasing spectra during the bidirectional tuning processes of the floating quasi-disk microlaser. (b) Optical images of the excited microlaser with respect to the spectra. ${ }^{[15]}$ 


\subsection{Water-soluble organic compound sensing}

The floating quasi-disk microlasers can be further exploited as sensitive sensors to detect the water-soluble polar chemicals. As shown in Fig. 5.10 (a), another capillary tube was added to the microfluidic system to deliver the ethanol, which was water-soluble and selected as the target chemical, to the liquid environment. Although the ethanol molecules have a polarity which is smaller than the surfactants, they can still attract the water molecules and reduce the surface tension of water. Therefore, the cavity size of the floating quasi-disk microlasers is decreased from $205 \mu \mathrm{m}$ to $180 \mu \mathrm{m}$ with the ethanol concentration increased from 0 to $1.007 \times 10^{-3} \mathrm{~mol} \cdot \mathrm{mL}^{-1}$ (Fig. 5.10 (b) and (c)). Meanwhile, the lasing spectrum is observed to be redshifted around 4 THz (Fig. 5.10 (d)). As the shift of the spectrum is larger than FSR, which is different from the case introduced in Chapter 4, the calculation of the sensitivity should be based on the FSR variation instead of the shift of a single mode. The enlarged spectra in Fig. 5.10 (d) reveals a $0.02 \mathrm{THz}$-change in the FSR, which results in the FSR sensitivity of $19.85 \mathrm{THz} /\left(\mathrm{mol} \bullet \mathrm{mL}^{-1}\right)$. It is expected that the sensing mechanism is applicable to all the other polar chemicals that are water-soluble and do not react with the epoxy resin and the surfactants in the soap water. In addition, the sensitivity of the floating quasi-disk microlasers, which is proportional to the polarity of the target chemical, can be further improved with other water-soluble polar chemicals. 


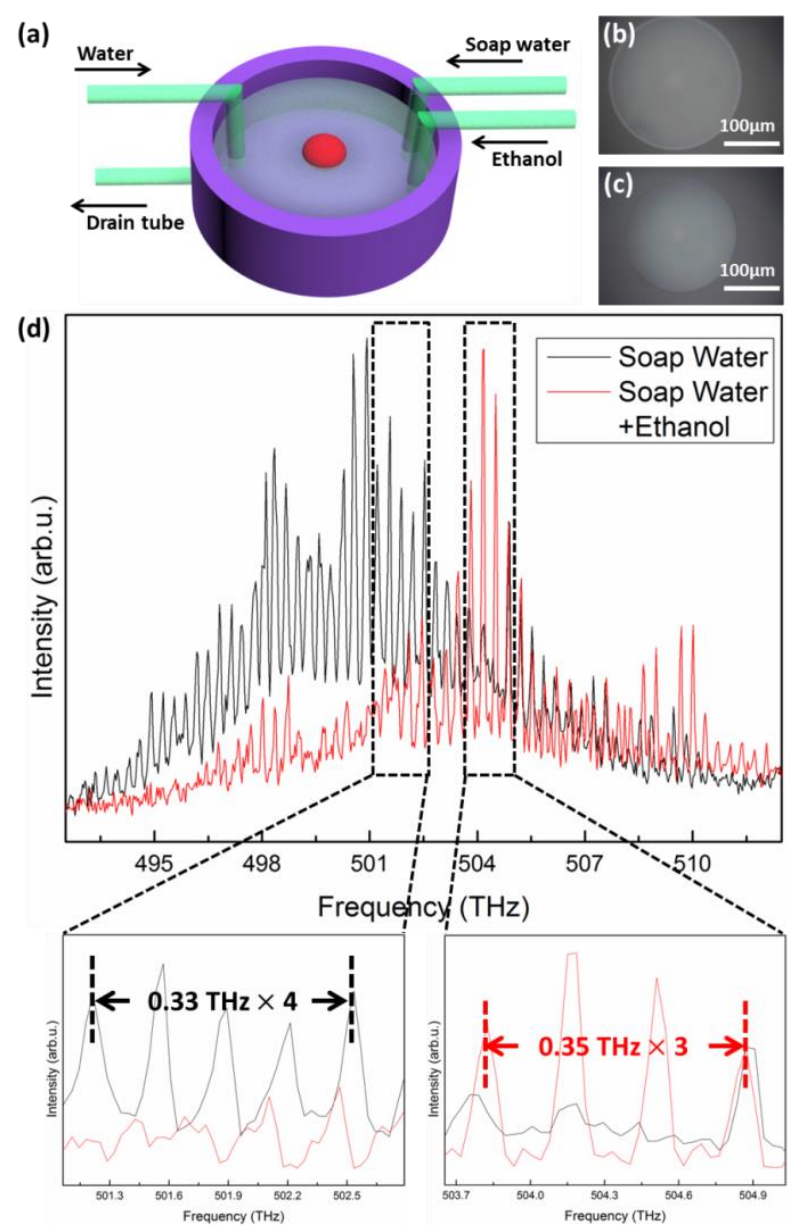

Figure 5.10. Sensing of the water-soluble polar chemicals.

(a) The microfluidic system with an extra capillary tube for ethanol delivery. (b) The floating quasi-disk microlaser without ethanol in the environment. (c) The same microlaser with a small amount of ethanol delivered into the liquid environment. $(d)$ Spectrum shift of the microlaser for ethanol sensing. The enlarged spectra indicate the measured FSR for the two statuses. ${ }^{[15]}$

\subsection{Summary and outlook}

In conclusion, the floating quasi-disk microlasers are inkjet-printed on the water surface by employing the dipping technique of the commercial microplotter. High-quality WGM lasing with the $Q$ factors around 5000 is achieved. Meanwhile, it is found that by engineering the surface tension of the liquid environment via the surfactants, the size of the floating quasi-disk microlasers can be reversibly tuned, which results in the bidirectional shift of the lasing spectra. In addition, the 
water-soluble polar chemical exemplified by ethanol, which does not react with the cavity materials and the environmental surfactants, is detected with the floating quasi-disk microlasers with the FSR sensitivity as high as $19.85 \mathrm{THz} /\left(\mathrm{mol} \bullet \mathrm{mL}^{-1}\right)$. This work not only proposes a novel, flexible and stable WGM microlaser in the liquid environment, but also presents a new method to bi-directionally and reconfigurably tune the resonant peaks with a relatively large tuning range, which opens up potential applications in microfluidics and bio/chemical sensing.

Further improvements of the floating quasi-disk microlasers can be based on the enhancements of the reliability and sensitivity. Although the measured microlasers are fixed at the center of the liquid environment due to the surface-tension-induced large curvature of the liquid surface in the small container of the home-made microfluidic system, the unavoidable Brownian motions of the water molecules still perturb the recorded spectra. Thus, minimization of the effects brought by the Brownian motions should be addressed in future studies of the floating quasi-disk microlasers in order to increase the sensing reliability. Furthermore, the improvements in the sensitivity, not only by choosing chemicals with higher polarities than the ethanol molecules, but also through enhancing the intrinsic qualities of the laser cavity via advanced materials and/or fabrication techniques, are needed. 


\section{Conclusions and Perspectives}

\subsection{Conclusion}

In this thesis, the significance and fundamentals of the microcavity and microlaser studies are firstly addressed, including the classification of microcavities and microlasers, the abundant applications, principles of dye lasers, as well as the relevant rate equations. Then the WGM basics are emphasized by deriving the asymptotic formulae, the characteristic equations, and by introducing the important WGM parameters. In order to get a deep understanding of the WGM microcavities and microlasers, which is the core of the works in this thesis, the relevant developments are reviewed from the aspect of material evolution. Brief and representative examples for the glassy candidates, semiconductor-based and soft-approached WGM microcavities and microlasers, together with the current challenges in this field, are given in the last part of Chapter 1.

The properties of flexible, tunable, cost-efficient, which are hard to achieve in the conventional solid candidates due to the rigid nature of the materials, are significant for further broadening the applications for WGM microcavities and microlasers. Therefore, after the introduction of the applied experimental techniques in Chapter 2, three works which enhance the flexibility of the soft-approached WGM microlasers with different geometries are presented. The inkjet-printed hemispherical microlaser introduced in Chapter 3 focuses on tuning the lasing peaks via the gain medium modification. By utilizing the nonradiative FRET processes between the donor and 
acceptor molecules, low-threshold lasing with single color and multi colors can be achieved and potentially applied in display and illumination. In Chapter 4, the resonant wavelengths of the microfiber array buried in PDMS matrix are tuned via the enlarged cavity size induced by external forces, which can be further exploited as sensitive strain sensors. The novel floating quasi-disk microlasers in Chapter 5 are sensitive to the liquid environment. Thus, by manipulating the concentration of the surfactants in the liquid surrounding, the diameters of the microlasers, as well as the lasing peaks, can be tuned, which is promising in the fields of microfluidic sensing and bio/chemical sensing. In addition, the enhanced flexibility of the proposed soft-approached WGM microlasers is not only reflected in the elastic tuning of the lasing spectra, but also exemplified by the advanced and cost-efficient fabrication techniques, which enables the demonstrated tunable microlasers to be competitive in potential applications such as display and sensing.

Although the three works presented in this thesis show encouraging improvements comparing with previous reports in this field, there are still some aspects that need to be improved. The detailed suggestions are given in the summary and outlook sections in Chapter 3, 4, 5, respectively. On the other hand, enhancing the flexibility, or tuning the resonant wavelengths of the WGM microcavities and microlasers, is only a branch of the microcavity studies. Abundant works can be done in other aspects to investigate the intrinsic properties of the WGM microcavities and microlasers and further broaden the relevant applications. 


\subsection{Future directions}

\subsubsection{High-efficiency coupling of the polymer waveguides and WGM}

microlasers

The evanescent field of a typical WGM microlaser is normally omnidirectional and requires waveguides to couple it in and out. Generally, the prisms, the polished fibers, and the tapered fibers are the three widely applied waveguides for WGM coupling. ${ }^{[6]}$ Comparing with the prisms used in early experiments, which can reach the coupling efficiency around $80 \%,{ }^{[173-175]}$ the polished fibers are more compatible with fiber optics. ${ }^{[176]}$ On the other hand, the tapered fibers, which are fabricated by stretching the softened commercial optical fibers under mega-thermal environments, can dramatically improve the coupling efficiency to $99.99 \%$ and show relatively high ideality due to the wavelength-scaled tapered regions. ${ }^{[177-181]}$ However, as the coupling efficiency critically relies on the distance between the coupler and the WGM microcavity, sophisticated setups are needed, which increases the cost and hinder the applications. In contrast, by taking advantage of the flexibility of the polymer materials as well as the precise dipping and drawing techniques of the microplotter, the hemispherical microlasers and waveguides can be simply plotted and drawn on the substrate with controllable cavity-waveguide distances (Fig. 6.1 (a)). In addition, multicolor lasing or even white-light lasing can be achieved by coupling the emission of different dye-doped hemispheres via the same waveguide. Similar results are also expected with the coupled microfiber geometry shown in Fig. 6.1 (b), where the circular cross-sections of the active microfibers are utilized as WGM lasing planes 
while the underlying passive microfiber is used as the waveguide. All the fibers are drawn by the spiral drawing technique with predetermined numbers of microlasers of each color, so the output color can be well-controlled. Detailed studies of the intrinsic energy transfer processes, as well as the investigation of the coupling efficiencies, are also needed for extending the applications to display and illumination.
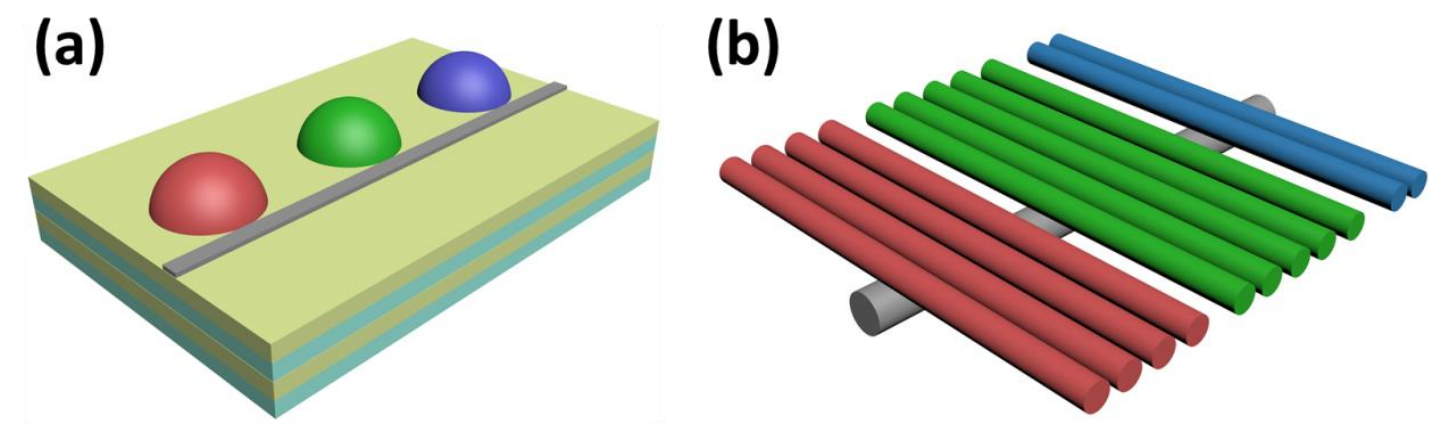

Figure 6.1. Coupling of the polymer waveguides and WGM microlasers.

(a) Multicolor emission from different dye-doped hemispheres and a polymer waveguide which is controllably drawn on the substrate. (b) Coupled microfiber geometry. The emission with various colors from the WGM microlasers on the top is guided via the underlying passive polymer fiber.

\subsubsection{Unidirectional emission of the WGM microlasers}

Instead of external waveguides, another possible solution for the problem of omnidirectional emitted WGMs is to realize the unidirectional emission of the microlasers so that the signals can be collected in free space with high efficiency. ${ }^{[182]}$ Generally, existing works on unidirectional emitted WGM microlasers are based on four aspects. One approach is the scatter-induced unidirectional emission where the scatterer can be located inside, outside or at the boundary of the microcavity. ${ }^{[182-184]}$ Another method is to purposefully deform the circular cavity to induce optical chaos, which changes the original evanescent leakage of the confined light to refractive 
escape because the conditions of the total internal reflection are no longer satisfied at some points of the deformed cavity. Typical examples are the spiral, triangular, limacon, D-shaped, and short-egg cavities. ${ }^{[185-191]}$ In addition to the emission in the horizontal WGM plane, directional vertical emission can also be achieved via the diffractions induced by the periodic gratings inside the cavity. ${ }^{[192]}$ Furthermore, combining the optical devices to guide the emitted WGM signals is also an applicable way to enhance the efficiency of collection. ${ }^{[193]}$

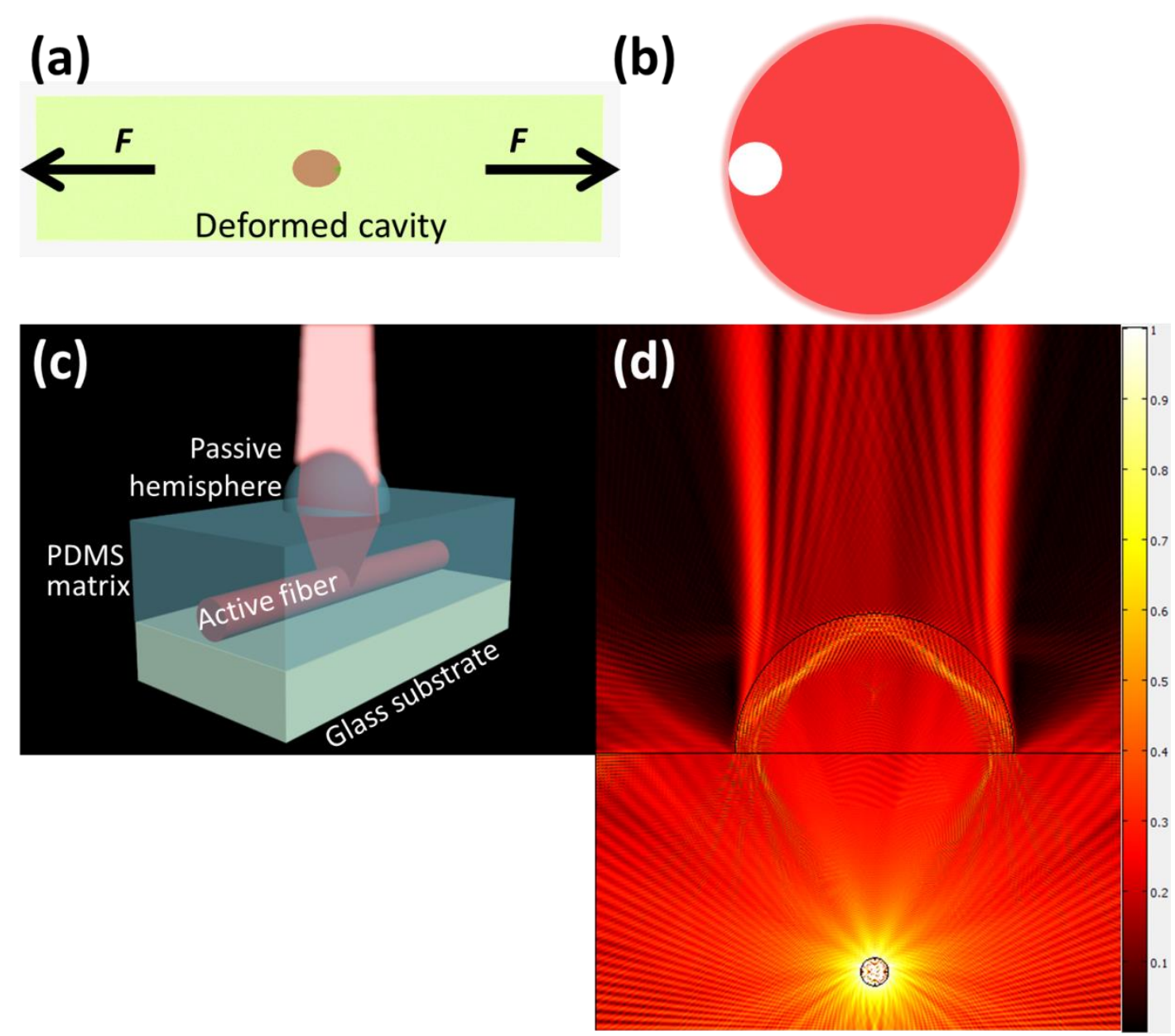

Figure 6.2. Directional emission from the polymer WGM microlasers.

(a) Deformed WGM plane which induces directional lasing with external forces applied to the surrounding polymer matrix. (b) Scatter-induced directional emission with an air bubble inside a polymer microsphere. (c) Combination of an active microfiber laser with the passive hemispherical lens. (d) Simulation of the combined geometry in (c). 
Engineering unidirectional emitted WGM microcavities and microlasers with high quality factors and small divergence angles is significant for practical applications. However, the reported works are mostly based on semiconductors with complex fabrication setups, sacrificed $Q$ factors, and unsatisfactory divergence angles. ${ }^{[182]}$ Indeed, the flexible polymer-material-based WGM configurations have great potentials to solve these challenges, and are largely unexplored. As exemplified in Fig. 6.2 (a), instead of photolithography, the cavity shape can be easily deformed and adjusted via the external forces applied to the surrounding polymer matrix due to the intrinsic soft nature of the polymers. Fig. 6.2 (b) illustrates the possibility to achieve scatter-induced unidirectional emission via an air bubble inside a polymer microsphere. The combination of an active microfiber laser with the passive hemispherical lens on the top is demonstrated in Fig. 6.2 (c) and (d). The calculated divergence angle can be reduced to less than $10^{\circ}$ with appropriate distance between the polymer microfiber and the hemisphere.

\subsubsection{Symmetry breaking and its applications in soft-approached WGM microcavities and microlasers}

Initially, the parity-time (PT) symmetry is applied to regulate the non-Hermitian Hamiltonians in quantum mechanics. ${ }^{[194]}$ Due to the similarity of Schrödinger equation and the wave equation, the PT symmetry is introduced to optics. ${ }^{[195,196]}$ Recently, light manipulation by PT-symmetry breaking in a coupled WGM microlaser system has been a hot topic. ${ }^{[197-200]}$ With one microlaser providing gain and the other 
one experiencing loss, selective breaking of the PT-symmetric system can lead to the enhancement of a single mode even though there are other adjacent modes in the amplification region of the gain medium; or implement switchable non-reciprocal optical isolation, which can be utilized in on-chip light control devices such as optical diodes. Unfortunately, due to the relatively low refractive indices, the $Q$ factors of the polymer WGM microcavities and microlasers are lower than the semiconductor ones, which make the study of PT symmetry with polymer materials difficult. Nevertheless, it is still worth to try as the flexibility of the polymer materials can not only results in interesting physical phenomena, but also abundant practical applications in this field.

\subsubsection{Electrically excited soft microlasers}

The current interrogation system of the polymer WGM microlasers requires a pulsed laser with high power density, which is inconvenient due to the large size of the excitation laser and the complication in adjusting the optical paths. Therefore, replacing the pulsed laser with the electrically driven LEDs can dramatically reduce the cost and complexity of the soft-approached WGM microlasers and make them more practical for on-chip applications. In order to achieve the indirect electrical pumping of the polymer microlasers, the LEDs need to be embedded in the substrates, which exhibit high transmittance to the light emitted by the LEDs and high reflectivity to the emission from the microlasers. The most important issue is the power density provided by the excitation LEDs. Although several works have achieved LED excitation of organic lasers, ${ }^{[201,202]}$ the current LED energy density is 
still too low compared to the lasing thresholds of the dye-doped polymer WGM microlasers. ${ }^{[202]}$ Therefore, optimizing the concentration of the organic dyes and increasing the $Q$ factor through advanced fabrication techniques that induce less imperfection are important for reducing the lasing threshold. In addition, the development of the LED industry is also progressing fast so it is possible that with the decrease of the lasing threshold and the increase of the excitation power density, electrically pumped soft WGM microlaser could be realized in the future. 


\section{Bibliography}

[1] T. H. Maiman, Stimulated optical radiation in ruby, Nature, 187, 4736 (1960).

[2] I. D. Samuel, E. B. Namdas, G. A. Turnbull, How to recognize lasing, Nature Photonics, 3, 546 (2009).

[3] Y. Ohba, M. Ishikawa, H. Sugawara, M. Yamamoto, T. Nakanisi, Growth of high-quality InGaAIP epilayers by MOCVD using methyl metalorganics and their application to visible semiconductors lasers, Journal of Crystal Growth, 77, 374 (1986).

[4] A. Ikesue, T. Kinoshita, K. Kamata, K. Yoshida, Fabrication and optical properties of high - Performance polycrystalline Nd: YAG ceramics for solid - State lasers, Journal of the American Ceramic Society, 78, 1033 (1995).

[5] H. Zhu, Y. Fu, F. Meng, X. Wu, Z. Gong, Q. Ding, M. V. Gustafsson, M. T. Trinh, S. Jin, X. Zhu, Lead halide perovskite nanowire lasers with low lasing thresholds and high quality factors, Nature materials, 14, 636 (2015).

[6] S. Yang, Y. Wang, H. D. Sun, Advances and prospects for whispering gallery mode microcavities, Advanced Optical Materials, 3, 1136 (2015).

[7] H. J. Caulfield, S. Dolev, Why future supercomputing requires optics, Nature Photonics, 4, 261 (2010).

[8] C. V. Poulton, X. Zeng, M. T. Wade, M. A. Popović, Channel add-drop filter based on dual photonic crystal cavities in push-pull mode, Optics letters, 40, 4206 (2015).

[9] X. Hu, P. Jiang, C. Ding, H. Yang, Q. Gong, Picosecond and low-power all-optical switching based on an organic photonic-bandgap microcavity, Nature Photonics, 2 , 185 (2008).

[10] R. Chen, V. D. Ta, H. D. Sun, Single mode lasing from hybrid hemispherical microresonators, Scientific reports, 2, 244 (2012).

[11] M. T. Hill, H. J. Dorren, T. De Vries, X. J. Leijtens, J. H. Den Besten, B. Smalbrugge, Y.-S. Oei, H. Binsma, G.-D. Khoe, M. K. Smit, A fast low-power optical memory based on coupled micro-ring lasers, Nature, 432, 206 (2004).

[12] S. Koehl, A. Liu, M. Paniccia, Integrated silicon photonics: Harnessing the data explosion, Optics and Photonics News, 22, 24 (2011).

[13] S. Chan, Y. Li, L. J. Rothberg, B. L. Miller, P. M. Fauchet, Nanoscale silicon microcavities for biosensing, Materials Science and Engineering: C, 15, 277 (2001).

[14] V. Mulloni, L. Pavesi, Porous silicon microcavities as optical chemical sensors, Applied Physics Letters, 76, 2523 (2000).

[15] S. Yang, Y. Wang, R. Chen, T. He, H. V. Demir, H. D. Sun, Reconfigurable Liquid Whispering Gallery Mode Microlasers, Scientific reports, 6, 27200 (2016).

[16] A. M. Armani, R. P. Kulkarni, S. E. Fraser, R. C. Flagan, K. J. Vahala, Label-free, single-molecule detection with optical microcavities, science, 317, 783 (2007).

[17] M. R. Lee, P. M. Fauchet, Nanoscale microcavity sensor for single particle detection, Optics letters, 32, 3284 (2007).

[18] A. Imamog, D. D. Awschalom, G. Burkard, D. P. DiVincenzo, D. Loss, M. Sherwin, A. Small, Quantum information processing using quantum dot spins and 
cavity QED, Physical Review Letters, 83, 4204 (1999).

[19] J. Li, X. Zhan, C. Ding, D. Zhang, Y. Wu, Enhanced nonlinear optics in coupled optical microcavities with an unbroken and broken parity-time symmetry, Physical Review A, 92, 043830 (2015).

[20] Y. Wang, S. Yang, H. Yang, H. D. Sun, Quaternary Alloy Quantum Dots: Toward Low - Threshold Stimulated Emission and All - Solution - Processed Lasers in the Green Region, Advanced Optical Materials, 3, 652 (2015).

[21] Y. Wang, K. E. Fong, S. Yang, V. D. Ta, Y. Gao, Z. Wang, V. Nalla, H. V. Demir, H. D. Sun, Unraveling the ultralow threshold stimulated emission from $\mathrm{CdZnS} / \mathrm{ZnS}$ quantum dot and enabling high - Q microlasers, Laser \& Photonics Reviews, 9, 507 (2015).

[22] K. J. Vahala, Optical microcavities, Nature, 424, 839 (2003).

[23] L. Rayleigh, CXII. The problem of the whispering gallery, The London, Edinburgh, and Dublin Philosophical Magazine and Journal of Science, 20, 1001 (1910).

[24] L. Rayleigh, On the propagation of waves through a stratified medium, with special reference to the question of reflection, Proceedings of the Royal Society of London. Series A, 86, 207 (1912).

[25] R. Richtmyer, Dielectric resonators, Journal of Applied Physics, 10, 391 (1939).

[26] C. Garrett, W. Kaiser, W. Bond, Stimulated emission into optical whispering modes of spheres, Physical Review, 124, 1807 (1961).

[27] J. Ward, O. Benson, WGM microresonators: sensing, lasing and fundamental optics with microspheres, Laser \& Photonics Reviews, 5, 553 (2011).

[28] V. D. Ta, R. Chen, H. D. Sun, Self - Assembled Flexible Microlasers, Advanced Materials, 24, (2012).

[29] C. Zhang, C. L. Zou, Y. Yan, C. Wei, J. M. Cui, F. W. Sun, J. Yao, Y. S. Zhao, Self - Assembled Organic Crystalline Microrings as Active Whispering - Gallery Mode Optical Resonators, Advanced Optical Materials, 1, 357 (2013).

[30] T. J. Kippenberg, J. Kalkman, A. Polman, K. J. Vahala, Demonstration of an erbium-doped microdisk laser on a silicon chip, Physical Review A, 74, 051802 (2006).

[31] D. Armani, B. Min, A. Martin, K. J. Vahala, Electrical thermo-optic tuning of ultrahigh-Q microtoroid resonators, Applied Physics Letters, 85, 5439 (2004).

[32] V. D. Ta, R. Chen, H. D. Sun, Coupled polymer microfiber lasers for single mode operation and enhanced refractive index sensing, Advanced Optical Materials, 2, 220 (2014).

[33] M. Asano, Y. Takeuchi, W. Chen, Ş. K. Özdemir, R. Ikuta, N. Imoto, L. Yang, T. Yamamoto, Observation of optomechanical coupling in a microbottle resonator, Laser \& Photonics Reviews, 10, 603 (2016).

[34] S. Berneschi, D. Farnesi, F. Cosi, G. N. Conti, S. Pelli, G. Righini, S. Soria, High Q silica microbubble resonators fabricated by arc discharge, Optics letters, 36, 3521 (2011).

[35] V. Zamora, A. Díez, M. V. Andrés, B. Gimeno, Cylindrical optical microcavities: Basic properties and sensor applications, Photonics and 
Nanostructures-Fundamentals and Applications, 9, 149 (2011).

[36] A. A. Savchenkov, V. S. Ilchenko, A. B. Matsko, L. Maleki, High-order tunable filters based on a chain of coupled crystalline whispering gallery-mode resonators, IEEE photonics technology letters, 17, 136 (2005).

[37] H. Tapalian, J.-P. Laine, P. Lane, Thermooptical switches using coated microsphere resonators, IEEE photonics technology letters, 14, 1118 (2002).

[38] L. Liu, R. Kumar, K. Huybrechts, T. Spuesens, G. Roelkens, E.-J. Geluk, T. de Vries, P. Regreny, D. Van Thourhout, R. Baets, An ultra-small, low-power, all-optical flip-flop memory on a silicon chip, Nature Photonics, 4, 182 (2010).

[39] J. Yang, L. J. Guo, Optical sensors based on active microcavities, IEEE Journal of selected topics in quantum electronics, 12, 143 (2006).

[40] D. Livshits, A. Y. Egorov, H. Riechert, $8 \mathrm{~W}$ continuous wave operation of InGaAsN lasers at $1.3 \mu \mathrm{m}$, Electronics Letters, 36, 1381 (2000).

[41] B. B. Snavely, Flashlamp-excited organic dye lasers, Proceedings of the IEEE, 57, 1374 (1969).

[42] F. J. Duarte, P. Kelley, L. W. Hillman, P. F. Liao, Dye laser principles, Academic Press, 1990.

[43] H. Yokoyama, S. Brorson, Rate equation analysis of microcavity lasers, Journal of Applied Physics, 66, 4801 (1989).

[44] C. Lam, P. T. Leung, K. Young, Explicit asymptotic formulas for the positions, widths, and strengths of resonances in Mie scattering, JOSA B, 9, 1585 (1992).

[45] R. Chen, V. D. Ta, H. D. Sun, Bending-induced bidirectional tuning of whispering gallery mode lasing from flexible polymer fibers, ACS Photonics, 1, 11 (2014).

[46] S. K. Tang, R. Derda, Q. Quan, M. Lončar, G. M. Whitesides, Continuously tunable microdroplet-laser in a microfluidic channel, Optics express, 19, 2204 (2011).

[47] V. D. Ta, R. Chen, D. Nguyen, H. D. Sun, Application of self-assembled hemispherical microlasers as gas sensors, Applied Physics Letters, 102, 031107 (2013).

[48] V. D. Ta, R. Chen, L. Ma, Y. J. Ying, H. D. Sun, Whispering gallery mode microlasers and refractive index sensing based on single polymer fiber, Laser \& Photonics Reviews, 7, 133 (2013).

[49] A. Kavokin, J. J. Baumberg, G. Malpuech, F. P. Laussy, Microcavities, Oxford University Press, 2011.

[50] M. Gomilšek, Whispering gallery modes, University of Ljubljana, Ljubljana, Seminar, 2011.

[51] A. N. Oraevsky, Whispering-gallery waves, Quantum Electronics, 32, 377 (2002).

[52] M. L. Gorodetsky, A. A. Savchenkov, V. S. Ilchenko, Ultimate Q of optical microsphere resonators, Optics letters, 21, 453 (1996).

[53] G. Righini, Y. Dumeige, P. Féron, M. Ferrari, G. Nunzi Conti, D. Ristic, S. Soria, Whispering gallery mode microresonators: fundamentals and applications, Rivista del Nuovo Cimento, 34, 435 (2011).

[54] E. Hecht, Optics, Pearson Education, 2016. 
[55] B. E. A. Saleh, M. C. Teich, Fundamentals of Photonics, Wiley, 2013.

[56] A. B. Matsko, Practical Applications of Microresonators in Optics and Photonics, CRC Press, 2009.

[57] A. A. Savchenkov, A. B. Matsko, V. S. Ilchenko, L. Maleki, Optical resonators with ten million finesse, Optics express, 15, 6768 (2007).

[58] Y. Ido, Y. Masumoto, F. Sasaki, M. Mori, S. Haraichi, S. Hotta, Reduced lasing threshold in thiophene/phenylene co-oligomer crystalline microdisks, Applied physics express, 3, 012702 (2009).

[59] Y. X. Zhang, X. Y. Pu, K. Zhu, L. Feng, Threshold property of whispering-gallery-mode fiber lasers pumped by evanescent waves, Journal of the Optical Society of America B, 28, 2048 (2011).

[60] R. Slusher, A. Levi, U. Mohideen, S. McCall, S. Pearton, R. Logan, Threshold characteristics of semiconductor microdisk lasers, Applied Physics Letters, 63, 1310 (1993).

[61] V. Sandoghdar, F. Treussart, J. Hare, V. Lefevre-Seguin, J. M. Raimond, S. Haroche, Very low threshold whispering-gallery-mode microsphere laser, Physical Review A, 54, R1777 (1996).

[62] Y. F. Xiao, C. H. Dong, C. L. Zou, Z. F. Han, L. Yang, G. C. Guo, Low-threshold microlaser in a high-Q asymmetrical microcavity, Optics letters, 34, 509 (2009).

[63] X. Peng, F. Song, S. Jiang, N. Peyghambarian, M. Kuwata-Gonokami, L. Xu, Fiber-taper-coupled L-band $\mathrm{Er}^{3+}$-doped tellurite glass microsphere laser, Applied Physics Letters, 82, 1497 (2003).

[64] N. Kitamura, M. Makihara, T. Sato, M. Hamai, I. Mogi, S. Awaji, K. Watanabe, M. Motokawa, Glass spheres produced by magnetic levitation method, Journal of non-crystalline solids, 293, 624 (2001).

[65] N. Kitamura, M. Makihara, M. Hamai, T. Sato, I. Mogi, S. Awaji, K. Watanabe, M. Motokawa, Containerless melting of glass by magnetic levitation method, Japanese Journal of Applied Physics, 39, L324 (2000).

[66] G. N. Conti, A. Chiasera, L. Ghisa, S. Berneschi, M. Brenci, Y. Dumeige, S. Pelli, S. Sebastiani, P. Feron, M. Ferrari, Spectroscopic and lasing properties of $\mathrm{Er}^{3+}$-doped glass microspheres, Journal of non-crystalline solids, 352, 2360 (2006).

[67] I. Bica, Formation of glass microspheres with rotating electrical arc, Materials Science and Engineering: B, 77, 210 (2000).

[68] S. Soria, S. Berneschi, M. Brenci, F. Cosi, G. Nunzi Conti, S. Pelli, G. C. Righini, Optical microspherical resonators for biomedical sensing, Sensors, 11, 785 (2011).

[69] P. Van Hoi, C. T. T. Ha, H. Q. Hung, Long-band emission of microsphere lasers based on erbium-doped sol-gel silica-alumina glasses, Applied Physics Letters, 87, (2005).

[70] M. Pöllinger, D. O’Shea, F. Warken, A. Rauschenbeutel, Ultrahigh-Q tunable whispering-gallery-mode microresonator, Physical Review Letters, 103, 053901 (2009).

[71] G. S. Murugan, J. S. Wilkinson, M. N. Zervas, Selective excitation of whispering gallery modes in a novel bottle microresonator, Optics express, 17, 11916 (2009).

[72] M. Sumetsky, Y. Dulashko, R. Windeler, Optical microbubble resonator, Optics 
letters, 35, 898 (2010).

[73] M. Sumetsky, Y. Dulashko, R. Windeler, Super free spectral range tunable optical microbubble resonator, Optics letters, 35, 1866 (2010).

[74] A. Nakagawa, S. Ishii, T. Baba, Photonic molecule laser composed of GaInAsP microdisks, Applied Physics Letters, 86, 041112 (2005).

[75] B. E. Little, J. Foresi, G. Steinmeyer, E. Thoen, S. Chu, H. Haus, E. Ippen, L. Kimerling, W. Greene, Ultra-compact Si-SiO 2 microring resonator optical channel dropping filters, IEEE photonics technology letters, 10, 549 (1998).

[76] D. Bachman, A. Tsay, V. Van, Negative coupling and coupling phase dispersion in a silicon quadrupole micro-racetrack resonator, Optics express, 23, 20089 (2015).

[77] J. Jager, P. Noé, E. Picard, V. Calvo, E. Delamadeleine, E. Hadji, Whispering gallery modes in Er-doped silicon-rich oxide toroidal microcavities on chip, Physica E: Low-dimensional Systems and Nanostructures, 41, 1127 (2009).

[78] A. H. Choi, Handbook of Optical Microcavities, CRC Press, 2014.

[79] M. Hossein-Zadeh, K. J. Vahala, Free ultra-high-Q microtoroid: a tool for designing photonic devices, Optics express, 15, 166 (2007).

[80] A. M. Armani, K. J. Vahala, Biological and chemical detection using ultra-high-Q toroidal microresonators, Biophys. J, 94, 29a (2007).

[81] C. L. Haynes, R. P. Van Duyne, Nanosphere lithography: a versatile nanofabrication tool for studies of size-dependent nanoparticle optics, The Journal of Physical Chemistry B, 105, 5599 (2001).

[82] K. Li, H. Choi, Air-spaced GaN nanopillar photonic band gap structures patterned by nanosphere lithography, Journal of Applied Physics, 109, 023107 (2011).

[83] W. Y. Fu, K. K. Y. Wong, H. Choi, Room temperature photonic crystal band-edge lasing from nanopillar array on GaN patterned by nanosphere lithography, Journal of Applied Physics, 107, 063104 (2010).

[84] W. Y. Fu, K. K. Y. Wong, H. Choi, Close-packed hemiellipsoid arrays: A photonic band gap structure patterned by nanosphere lithography, Applied Physics Letters, 95, 133125 (2009).

[85] K. Li, Z. Ma, H. Choi, Single-mode whispering gallery lasing from metal-clad GaN nanopillars, Optics letters, 37, 374 (2012).

[86] K. Li, Z. Ma, H. Choi, High-Q whispering-gallery mode lasing from nanosphere-patterned GaN nanoring arrays, Applied Physics Letters, 98, 071106 (2011).

[87] R. Chen, B. Ling, X. W. Sun, H. D. Sun, Room Temperature Excitonic Whispering Gallery Mode Lasing from High - Quality Hexagonal ZnO Microdisks, Advanced Materials, 23, 2199 (2011).

[88] S. Shopova, G. Farca, A. Rosenberger, W. Wickramanayake, N. Kotov, Microsphere whispering-gallery-mode laser using HgTe quantum dots, Applied Physics Letters, 85, 6101 (2004).

[89] G. C. Righini, F. Cosi, G. N. Conti, S. Pelli, S. Soria, E. Moser, Y. Jestin, M. Ferrari, P. Féron, A. Chiasera, Photonic properties and applications of glass micro and nanospheres, physica status solidi (a), 206, 898 (2009).

[90] D. Ristić, V. Holý, M. Ivanda, M. Marciuš, M. Buljan, O. Gamulin, K. Furić, M. 
Ristić, S. Musić, M. Mazzola, Surface characterization of thin silicon-rich oxide films, Journal of molecular structure, 993, 214 (2011).

[91] A. Beltaos, A. Meldrum, Whispering gallery modes in silicon-nanocrystal-coated silica microspheres, Journal of luminescence, 126, 607 (2007).

[92] S. Shibata, S. Ashida, H. Segawa, T. Yano, Coated microsphere as spherical cavity Raman laser, Journal of sol-gel science and technology, 40, 379 (2006).

[93] H. M. Tzeng, K. Wall, M. B. Long, R. K. Chang, Evaporation and condensation rates of liquid droplets deduced from structure resonances in the fluorescence spectra, Optics letters, 9, 273 (1984).

[94] S. X. Qian, J. B. Snow, H. M. Tzeng, R. K. Chang, Lasing droplets: highlighting the liquid-air interface by laser emission, science, 231, 486 (1986).

[95] H. B. Lin, A. Huston, B. Justus, A. J. Campillo, Some characteristics of a droplet whispering-gallery-mode laser, Optics letters, 11, 614 (1986).

[96] J. Schafer, J. P. Mondia, R. Sharma, Z. Lu, A. Susha, A. Rogach, L. Wang, Quantum dot microdrop laser, Nano letters, 8, 1709 (2008).

[97] H. Li, L. Lei, Q. Zeng, J. Shi, C. Luo, H. Ji, Q. Ouyang, Y. Chen, Laser emission from dye doped microspheres produced on a chip, Sensors and Actuators B: Chemical, 145, 570 (2010).

[98] A. Kiraz, A. Kurt, M. Dündar, A. Demirel, Simple largely tunable optical microcavity, Applied Physics Letters, 89, 081118 (2006).

[99] A. B. Theberge, F. Courtois, Y. Schaerli, M. Fischlechner, C. Abell, F. Hollfelder, W. T. Huck, Microdroplets in microfluidics: an evolving platform for discoveries in chemistry and biology, Angewandte Chemie International Edition, 49, 5846 (2010).

[100] A. Huebner, D. Bratton, G. Whyte, M. Yang, C. Abell, F. Hollfelder, Static microdroplet arrays: a microfluidic device for droplet trapping, incubation and release for enzymatic and cell-based assays, Lab on a Chip, 9, 692 (2009).

[101] Y. Schaerli, R. C. Wootton, T. Robinson, V. Stein, C. Dunsby, M. A. Neil, P. M. French, A. J. demello, C. Abell, F. Hollfelder, Continuous-flow polymerase chain reaction of single-copy DNA in microfluidic microdroplets, Analytical chemistry, 81, 302 (2008).

[102] C. J. Ma, L. Y. Ren, Y. P. Xu, Y. L. Wang, J. Liang, E. S. Qu, Design and fabrication of tapered microfiber waveguide with good optical and mechanical performance, Journal of Modern Optics, 61, 683 (2014).

[103] A. Camposeo, F. Di Benedetto, R. Stabile, A. A. Neves, R. Cingolani, D. Pisignano, Laser emission from electrospun polymer nanofibers, Small, 5, 562 (2009). [104] A. J. Das, C. Lafargue, M. Lebental, J. Zyss, K. Narayan, Three-dimensional microlasers based on polymer fibers fabricated by electrospinning, Applied Physics Letters, 99, 263303 (2011).

[105] D. O'Carroll, I. Lieberwirth, G. Redmond, Microcavity effects and optically pumped lasing in single conjugated polymer nanowires, Nature nanotechnology, 2 , 180 (2007).

[106] V. Chagulov, Polymer fiber waveguides, Quantum Electronics, 12, 1587 (1982).

[107] H. Ma, A. Y. Jen, L. R. Dalton, Polymer - Based Optical Waveguides: Materials, Processing, and Devices, Advanced Materials, 14, 1339 (2002). 
[108] W. E. Speas, A quantitative study of the changes produced in the absorption bands of certain organic fluorescent dye solutions by alterations of concentration and temperature, Physical Review, 31, 569 (1928).

[109] M. M. Mazumder, J. B. Gillespie, G. Chen, R. K. Chang, Wavelength shifts of dye lasing in microdroplets: effect of absorption change, Optics letters, 20, 878 (1995).

[110] R. M. Clegg, Fluorescence resonance energy transfer, Current opinion in biotechnology, 6, 103 (1995).

[111] P. R. Selvin, The renaissance of fluorescence resonance energy transfer, Nature structural biology, 7, 730 (2000).

[112] S. K. Tang, Z. Li, A. R. Abate, J. J. Agresti, D. A. Weitz, D. Psaltis, G. M. Whitesides, A multi-color fast-switching microfluidic droplet dye laser, Lab on a Chip, 9, 2767 (2009).

[113] M. Humar, M. Ravnik, S. Pajk, I. Muševič, Electrically tunable liquid crystal optical microresonators, Nature Photonics, 3, 595 (2009).

[114] Z. Cai, A. Chardon, H. Xu, P. Féron, G. M. Stéphan, Laser characteristics at $1535 \mathrm{~nm}$ and thermal effects of an Er: Yb phosphate glass microchip pumped by Ti: sapphire laser, Optics communications, 203, 301 (2002).

[115] S. Wemple, Refractive-index behavior of amorphous semiconductors and glasses, Physical Review B, 7, 3767 (1973).

[116] Z. Zhang, P. Zhao, P. Lin, F. Sun, Thermo-optic coefficients of polymers for optical waveguide applications, Polymer, 47, 4893 (2006).

[117] A. Tewary, M. J. Digonnet, M. L. Brongersma, J. Y. Sung, J. H. Shin, Silicon-nanocrystal-coated silica microsphere thermooptical switch, Society of Photo-Optical Instrumentation Engineers, 2006.

[118] R. Chen, V. D. Ta, H. D. Sun, Tuning whispering gallery mode lasing from self-assembled polymer droplets, Scientific reports, 3, 1362 (2013).

[119] S. Yang, Y. Wang, Y. Gao, T. He, R. Chen, H. V. Demir, H. D. Sun, Multicolor lasing prints, Applied Physics Letters, 107, 221103 (2015).

[120] F. Sajed, C. Rutherglen, All-printed and transparent single walled carbon nanotube thin film transistor devices, Applied Physics Letters, 103, 143303 (2013).

[121] M. Croce, E. Bond, A. Hoover, G. Kunde, W. Moody, M. Rabin, D. Bennett, J. Hayes-Wehle, V. Kotsubo, D. Schmidt, Integration of Radioactive Material with Microcalorimeter Detectors, Journal of Low Temperature Physics, 176, 1009 (2014).

[122] M. Saito, H. Shimatani, H. Naruhashi, Tunable whispering gallery mode emission from a microdroplet in elastomer, Optics express, 16, 11915 (2008).

[123] T. D. McDonald, C. Bayer, A. M. DeLee, E. Atchison, D. Widrig, B. Hutchens, K. C. Leonard, Rapid Characterization of Multi-Metallic Electrocatalysts for the Water Splitting Reactions Utilizing Printed Microelectrodes on a Chip, Journal of The Electrochemical Society, 163, H359 (2016).

[124] D. Martins, X. Wei, R. Levicky, Y. A. Song, Accelerating the Mass Transport of DNA Biomolecules Onto DNA Microarray for Enhanced Detection by Electrokinetic Concentration in a Microfluidic Chip, ASME 2016 5th International Conference on Micro/Nanoscale Heat and Mass Transfer, American Society of Mechanical Engineers, 
2016, pp. V001T01A007.

[125] H. Yasuda, C. Lamaze, K. Sakaoku, Effect of electrodeless glow discharge on polymers, Journal of Applied Polymer Science, 17, 137 (1973).

[126] J. Venables, C. Harland, Electron back-scattering patterns-A new technique for obtaining crystallographic information in the scanning electron microscope, Philosophical Magazine, 27, 1193 (1973).

[127] J. Goldstein, D. E. Newbury, P. Echlin, D. C. Joy, A. D. Romig Jr, C. E. Lyman, C. Fiori, E. Lifshin, Scanning electron microscopy and X-ray microanalysis: a text for biologists, materials scientists, and geologists, Springer Science \& Business Media, 2012.

[128] A. Bogner, G. Thollet, D. Basset, P. H. Jouneau, C. Gauthier, Wet STEM: A new development in environmental SEM for imaging nano-objects included in a liquid phase, Ultramicroscopy, 104, 290 (2005).

[129] C. F. Klingshirn, Semiconductor optics, Springer Science \& Business Media, 2012.

[130] Y. Wang, Optical study of colloidal quantum dots: toward efficient emission and lasing upon single-and multi-photon excitation, 2015.

[131] R. Chen, V. D. Ta, F. Xiao, Q. Zhang, H. D. Sun, Multicolor hybrid upconversion nanoparticles and their improved performance as luminescence temperature sensors due to energy transfer, Small, 9, 1052 (2013).

[132] S. Tan, X. Sun, H. Demir, S. DenBaars, Advances in the LED materials and architectures for energy-saving solid-state lighting toward "lighting revolution", IEEE Photonics Journal, 4, 613 (2012).

[133] J. Piprek, Efficiency droop in nitride - based light - emitting diodes, physica status solidi (a), 207, 2217 (2010).

[134] Y. Ding, Q. Yang, X. Guo, S. Wang, F. Gu, J. Fu, Q. Wan, J. Cheng, L. Tong, Nanowires/microfiber hybrid structure multicolor laser, Optics express, 17, 21813 (2009).

[135] D. J. Gardiner, S. M. Morris, P. J. Hands, C. Mowatt, R. Rutledge, T. D. Wilkinson, H. J. Coles, Paintable band-edge liquid crystal lasers, Optics express, 19, 2432 (2011).

[136] P. A. Elzinga, R. J. Kneisler, F. E. Lytle, Y. Jiang, G. B. King, N. M. Laurendeau, Pump/probe method for fast analysis of visible spectral signatures utilizing asynchronous optical sampling, Applied optics, 26, 4303 (1987).

[137] R. J. Nedumpara, K. Thomas, V. Jayasree, C. Girijavallabhan, V. Nampoori, P. Radhakrishnan, Study of solvent effect in laser emission from Coumarin 540 dye solution, Applied optics, 46, 4786 (2007).

[138] M. Saito, K. Koyama, Deformable microdroplet cavity fabricated by an inkjet method, Japanese Journal of Applied Physics, 49, 092501 (2010).

[139] D. Gardiner, W. K. Hsiao, S. Morris, P. Hands, T. Wilkinson, I. Hutchings, H. Coles, Printed photonic arrays from self-organized chiral nematic liquid crystals, Soft Matter, 8, 9977 (2012).

[140] M. Beutler, R. Heintzmann, Förster resonance energy transfer, Encyclopedic Reference of Genomics and Proteomics in Molecular Medicine, Springer, 2005, pp. 
594.

[141] R. Chen, Y. Shen, F. Xiao, B. Liu, G. Gurzadyan, Z. L. Dong, X. W. Sun, H. D. Sun, Surface Eu-treated $\mathrm{ZnO}$ nanowires with efficient red emission, The Journal of Physical Chemistry C, 114, 18081 (2010).

[142] S. Crooker, J. Hollingsworth, S. Tretiak, V. I. Klimov, Spectrally resolved dynamics of energy transfer in quantum-dot assemblies: Towards engineered energy flows in artificial materials, Physical Review Letters, 89, 186802 (2002).

[143] F. Xiao, R. Chen, Y. Q. Shen, B. Liu, G. G. Gurzadyan, Z. L. Dong, Q. Y. Zhang, H. D. Sun, Infrared emission properties and energy transfer in $\mathrm{ZnO}-\mathrm{SiO}_{2}: \mathrm{Yb}^{3+}$ composites, Journal of Alloys and Compounds, 509, 7794 (2011).

[144] C. L. Zou, F. J. Shu, F. W. Sun, Z. J. Gong, Z. F. Han, G. C. Guo, Theory of free space coupling to high-Q whispering gallery modes, Optics express, 21, 9982 (2013).

[145] U. Ayaz, T. Ioppolo, M. Ötügen, Wall shear stress sensor based on the optical resonances of dielectric microspheres, Measurement science and technology, 22, 075203 (2011).

[146] I. Kang, M. J. Schulz, J. H. Kim, V. Shanov, D. Shi, A carbon nanotube strain sensor for structural health monitoring, Smart materials and structures, 15, 737 (2006).

[147] M. Manzo, T. Ioppolo, U. Ayaz, V. LaPenna, M. Ötügen, A photonic wall pressure sensor for fluid mechanics applications, Review of Scientific Instruments, 83, 105003 (2012).

[148] T. Yamada, Y. Hayamizu, Y. Yamamoto, Y. Yomogida, A. Izadi-Najafabadi, D. N. Futaba, K. Hata, A stretchable carbon nanotube strain sensor for human-motion detection, Nature nanotechnology, 6, 296 (2011).

[149] J. H. Choi, Y. S. No, J. P. So, J. M. Lee, K. H. Kim, M. S. Hwang, S. H. Kwon, H. G. Park, A high-resolution strain-gauge nanolaser, Nature communications, 7, (2016).

[150] B. Lee, Review of the present status of optical fiber sensors, Optical fiber technology, 9, 57 (2003).

[151] J. Zhou, Y. Gu, P. Fei, W. Mai, Y. Gao, R. Yang, G. Bao, Z. L. Wang, Flexible piezotronic strain sensor, Nano letters, 8, 3035 (2008).

[152] J. Albert, L. Y. Shao, C. Caucheteur, Tilted fiber Bragg grating sensors, Laser \& Photonics Reviews, 7, 83 (2013).

[153] T. Ioppolo, M. Kozhevnikov, V. Stepaniuk, M. V. Ötügen, V. Sheverev, Micro-optical force sensor concept based on whispering gallery mode resonators, Applied optics, 47, 3009 (2008).

[154] M. G. Allen, M. Mehregany, R. T. Howe, S. D. Senturia, Microfabricated structures for the insitu measurement of residual stress, young's modulus, and ultimate strain of thin films, Applied Physics Letters, 51, 241 (1987).

[155] F. M. Haran, J. K. Rew, P. D. Foote, A strain-isolated fibre Bragg grating sensor for temperature compensation of fibre Bragg grating strain sensors, Measurement science and technology, 9, 1163 (1998).

[156] E. J. Friebele, Fiber Bragg grating strain sensors: present and future applications in smart structures, Optics and Photonics News, 9, 33 (1998). 
[157] P. Wang, K. Xie, L. Shao, L. Yan, J. Xu, R. Chen, Longitudinal force measurement in continuous welded rail with bi-directional FBG strain sensors, Smart materials and structures, 25, 015019 (2015).

[158] C. Linslal, M. Kailasnath, S. Mathew, T. Nideep, P. Radhakrishnan, V. Nampoori, C. Vallabhan, Tuning whispering gallery lasing modes from polymer fibers under tensile strain, Optics letters, 41, 551 (2016).

[159] A. I. Gubin, A. A. Barannik, N. T. Cherpak, I. A. Protsenko, S. Pud, A. Offenhäusser, S. A. Vitusevich, Whispering-Gallery-Mode Resonator Technique With Microfluidic Channel for Permittivity Measurement of Liquids, IEEE transactions on microwave theory and techniques, 63, 2003 (2015).

[160] J. Wu, B. Liu, H. Zhang, B. Song, W. Lin, Y. Li, WGM Micro-fluidic-channel based on Reflection Type Fiber-tip-coupled Hollow-core PCFs, IEEE photonics technology letters, 28, 2565 (2016).

[161] V. Dantham, S. Holler, V. Kolchenko, Z. Wan, S. Arnold, Taking whispering gallery-mode single virus detection and sizing to the limit, Applied Physics Letters, 101, 043704 (2012).

[162] A. Yurt, G. G. Daaboul, J. H. Connor, B. B. Goldberg, M. S. Ünlü, Single nanoparticle detectors for biological applications, Nanoscale, 4, 715 (2012).

[163] H. Yokoyama, Liquid crystals: Tunable whispers, Nature Photonics, 3, 560 (2009).

[164] S. I. Shopova, Y. Sun, A. Rosenberger, X. Fan, Highly sensitive tuning of coupled optical ring resonators by microfluidics, Microfluidics and nanofluidics, $\mathbf{6}$, 425 (2009).

[165] A. Barucci, F. Baldini, S. Berneschi, F. Cosi, A. Giannetti, G. N. Conti, S. Soria, S. Tombelli, C. Trono, D. Farnesi, Whispering Gallery Modes Microresonators for Sensing and Biosensing Applications, Sensors, Springer, 2015, pp. 183.

[166] M. Oxborrow, Traceable 2-D finite-element simulation of the whispering-gallery modes of axisymmetric electromagnetic resonators, IEEE transactions on microwave theory and techniques, 55, 1209 (2007).

[167] X. Y. Hua, M. J. Rosen, Dynamic surface tension of aqueous surfactant solutions: I. Basic paremeters, Journal of Colloid and Interface Science, 124, 652 (1988).

[168] K. Holmberg, B. Jönsson, B. Kronberg, B. Lindman, Surfactants and polymers in aqueous solution, Journal of Synthetic Lubrication, 20, 367 (2004).

[169] I. Karatzas, S. Shreve, Brownian motion and stochastic calculus, Springer Science \& Business Media, 2012.

[170] J. D. Eversole, P. Leung, S. Liu, H. B. Lin, A. Huston, K. Young, A. Campillo, High-precision identification of morphology-dependent resonances in optical processes in microdroplets, JOSA B, 10, 1955 (1993).

[171] H. B. Lin, J. D. Eversole, A. J. Campillo, Spectral properties of lasing microdroplets, JOSA B, 9, 43 (1992).

[172] T. Kippenberg, S. Spillane, K. Vahala, Demonstration of ultra-high-Q small mode volume toroid microcavities on a chip, Applied Physics Letters, 85, 6113 (2004). 
[173] A. B. Matsko, V. S. Ilchenko, Optical resonators with whispering gallery modes I: basics, IEEE J. Sel. Top. Quantum Electron, 12, 3 (2006).

[174] F. Treussart, V. Ilchenko, J. F. Roch, J. Hare, V. Lefevre Seguin, J. M. Raimond, S. Haroche, Evidence for intrinsic Kerr bistability of high-Q microsphere resonators in superfluid helium, The European Physical Journal D-Atomic, Molecular, Optical and Plasma Physics, 1, 235 (1998).

[175] D. Rowland, J. Love, Evanescent wave coupling of whispering gallery modes of a dielectric cylinder, IEE Proceedings J-Optoelectronics, 140, 177 (1993).

[176] V. S. Ilchenko, X. S. Yao, L. Maleki, Pigtailing the high-Q microsphere cavity: a simple fiber coupler for optical whispering-gallery modes, Optics letters, 24, 723 (1999).

[177] J.-P. Laine, Design and applications of optical microsphere resonators, Helsinki University of Technology, 2003.

[178] M. Cai, O. Painter, K. J. Vahala, Observation of critical coupling in a fiber taper to a silica-microsphere whispering-gallery mode system, Physical Review Letters, $\mathbf{8 5}$, 74 (2000).

[179] A. Matsko, A. Savchenkov, D. Strekalov, V. Ilchenko, L. Maleki, Review of applications of whispering-gallery mode resonators in photonics and nonlinear optics, IPN Progress Report, 42, (2005).

[180] M. Cai, K. Vahala, Highly efficient hybrid fiber taper coupled microsphere laser, Optics letters, 26, 884 (2001).

[181] X. Peng, F. Song, S. Jiang, N. Peyghambarian, M. Kuwata Gonokami, L. Xu, Fiber-taper-coupled L-band $\mathrm{Er}^{3+}$-doped tellurite glass microsphere laser, Applied Physics Letters, 82, 1497 (2003).

[182] X. F. Jiang, C. L. Zou, L. Wang, Q. Gong, Y. F. Xiao, Whispering - gallery microcavities with unidirectional laser emission, Laser \& Photonics Reviews, 10, 40 (2016).

[183] Q. J. Wang, C. Yan, N. Yu, J. Unterhinninghofen, J. Wiersig, C. Pflügl, L. Diehl, T. Edamura, M. Yamanishi, H. Kan, Whispering-gallery mode resonators for highly unidirectional laser action, Proceedings of the National Academy of Sciences, 107, 22407 (2010).

[184] J. Wiersig, M. Hentschel, Unidirectional light emission from high-Q modes in optical microcavities, Physical Review A, 73, 031802 (2006).

[185] J. Wiersig, M. Hentschel, Combining directional light output and ultralow loss in deformed microdisks, Physical Review Letters, 100, 033901 (2008).

[186] G. Chern, H. Tureci, A. D. Stone, R. Chang, M. Kneissl, N. Johnson, Unidirectional lasing from InGaN multiple-quantum-well spiral-shaped micropillars, Applied Physics Letters, 83, 1710 (2003).

[187] M. Kurdoglyan, S. Y. Lee, S. Rim, C. M. Kim, Unidirectional lasing from a microcavity with a rounded isosceles triangle shape, Optics letters, 29, 2758 (2004).

[188] M. Hentschel, Q. J. Wang, C. Yan, F. Capasso, T. Edamura, H. Kan, Emission properties of electrically pumped triangular shaped microlasers, Optics express, 18, 16437 (2010).

[189] B. Redding, A. Cerjan, X. Huang, M. L. Lee, A. D. Stone, M. A. Choma, H. 
Cao, Low spatial coherence electrically pumped semiconductor laser for speckle-free full-field imaging, Proceedings of the National Academy of Sciences, 112, 1304 (2015).

[190] M. Schermer, S. Bittner, G. Singh, C. Ulysse, M. Lebental, J. Wiersig, Unidirectional light emission from low-index polymer microlasers, Applied Physics Letters, 106, 101107 (2015).

[191] T. Ben Messaoud, J. Zyss, Unidirectional laser emission from polymer-based spiral microdisks, Applied Physics Letters, 86, 241110 (2005).

[192] X. Cai, J. Wang, M. J. Strain, B. Johnson-Morris, J. Zhu, M. Sorel, J. L. O'Brien, M. G. Thompson, S. Yu, Integrated compact optical vortex beam emitters, science, 338, 363 (2012).

[193] T. Wienhold, S. Kraemmer, A. Bacher, H. Kalt, C. Koos, S. Koeber, T. Mappes, Efficient free-space read-out of WGM lasers using circular micromirrors, Optics express, 23, 1025 (2015).

[194] C. M. Bender, Introduction to PT-symmetric quantum theory Contemporary physics, 46, 277 (2005).

[195] L. Feng, Z. J. Wong, R. M. Ma, Y. Wang, X. Zhang, Single-mode laser by parity-time symmetry breaking, science, 346, 972 (2014).

[196] C. E. Rüter, K. G. Makris, R. El-Ganainy, D. N. Christodoulides, M. Segev, D. Kip, Observation of parity-time symmetry in optics, Nature Physics, 6, 192 (2010).

[197] H. Hodaei, M. A. Miri, M. Heinrich, D. N. Christodoulides, M. Khajavikhan, Parity-time-symmetric microring lasers, science, 346, 975 (2014).

[198] L. Chang, X. Jiang, S. Hua, C. Yang, J. Wen, L. Jiang, G. Li, G. Wang, M. Xiao, Parity-time symmetry and variable optical isolation in active-passive-coupled microresonators, Nature Photonics, 8, 524 (2014).

[199] S. Phang, A. Vukovic, S. C. Creagh, T. M. Benson, P. D. Sewell, G. Gradoni, Parity-time symmetric coupled microresonators with a dispersive gain/loss, Optics express, 23, 11493 (2015).

[200] B. Peng, S. K. Ozdemir, F. Lei, F. Monifi, M. Gianfreda, G. L. Long, S. Fan, F. Nori, C. M. Bender, L. Yang, Nonreciprocal light transmission in parity-time-symmetric whispering-gallery microcavities, arXiv preprint arXiv:1308.4564, DOI (2013).

[201] G. Tsiminis, Y. Wang, A. L. Kanibolotsky, A. R. Inigo, P. J. Skabara, I. D. Samuel, G. A. Turnbull, Nanoimprinted Organic Semiconductor Laser Pumped by a Light - Emitting Diode, Advanced Materials, 25, 2826 (2013).

[202] Y. Yang, G. Turnbull, I. Samuel, Hybrid optoelectronics: A polymer laser pumped by a nitride light-emitting diode, Applied Physics Letters, 92, 163306 (2008). 